\title{
KATY BOUSSEL
}

\section{Opérateurs hypergéométriques réductibles : décompositions et groupes de Galois différentiels}

\author{
Annales de la faculté des sciences de Toulouse $6^{e}$ série, tome $5, \mathrm{n}^{\circ} 2$ \\ (1996), p. 299-362 \\ <http://www.numdam.org/item?id=AFST_1996_6_5_2_299_0>
}

(C) Université Paul Sabatier, 1996, tous droits réservés.

L'accès aux archives de la revue «Annales de la faculté des sciences de Toulouse » (http://picard.ups-tlse.fr/ annales/) implique l'accord avec les conditions générales d'utilisation (http://www.numdam.org/conditions). Toute utilisation commerciale ou impression systématique est constitutive d'une infraction pénale. Toute copie ou impression de ce fichier doit contenir la présente mention de copyright.

\section{Numdam}

Article numérisé dans le cadre du programme Numérisation de documents anciens mathématiques http://www.numdam.org/ 


\title{
Opérateurs hypergéométriques réductibles : décompositions et groupes de Galois différentiels ${ }^{(*)}$
}

\author{
KATY BousSEL ${ }^{(1)}$
}

RÉSUMÉ. - Nous classons les opérateurs hypergéométriques réductibles suivant, d'une part, leurs différentes décompositions dans l'anneau des opérateurs différentiels et, d'autre part, des conditions paramétriques obtenues sous forme algorithmique. Cette classification nous permet à partir des travaux antérieurs de N. Katz sur le cas irréductible de calculer la partie unipotente du groupe de Galois différentiel grâce à des méthodes de cohomologie galoisienne et des considérations sur les groupes affines.

\begin{abstract}
We classify the reducible hypergeometric operators according to their different decompositions in the ring of differential operators in the one hand and to their parameters in the other hand. Starting from the work of N. Katz on the irreducible case, we derive from this classification a computation of the unipotent part of the differential Galois group, based on Galois cohomology methods and on considerations on affine groups.
\end{abstract}

\section{Introduction}

Cet article comprend deux parties : la première est consacrée aux différentes décompositions à isomorphisme près d'un opérateur différentiel hypergéométrique réductible; et la deuxième, qui s'appuie sur la première, comporte l'étude du groupe de Galois différentiel de ces opérateurs dans deux cas significatifs.

(*) Reçu le 4 novembre 1993

(1) U.R.A. 763 "Problèmes diophantiens", Mathématiques, T. 45-46, 5ème étage, 4 place Jussieu, F-75252 Paris Cedex 05 (France) 
Rappelons que pour $q, p$ deux entiers $\geq 0$, on appelle opérateur hypergéométrique l'opérateur :

$$
L=L\left(\left\{\mu_{i}\right\}_{i},\left\{\nu_{j}\right\}_{j} ; p, q\right)=(-1)^{q-p} z \prod_{i=1, \ldots, p}\left(\theta+\mu_{i}\right)-\prod_{j=1, \ldots, q}\left(\theta+\nu_{j}-1\right)
$$

où $\theta=z(\mathrm{~d} / \mathrm{d} z)$; les $\mu_{i}$ et les $\nu_{j}$ étant des nombres complexes.

À la première section, nous classons les opérateurs réductibles. Les opérateurs irréductibles hypergéométriques ont été étudiés et classifiés par N. Katz [Ka], et aussi par F. Beukers, D. Brownawell et G.Heckmann [BBH]. Le paragraphe 1.1.2 rappelle certains de leurs résultats.

Le théorème 1.2.1 donne le lien entre le type de décomposition de l'opérateur et les paramètres. Ceci est complètement régi par les conditions $C(r, m, n)$ (déf. 1.2.3). Lorsque $r=m+n$, toutes les décompositions ont alors la même "forme". Les conditions $C(r, m, n)$ dépendent du nombre de paramètres $\mu_{i}$ et $\nu_{j}$ vérifiant $\mu_{i}-\nu_{j} \in \mathbb{Z}$ et du signe des différences $\mu_{i}-\nu_{j}$.

Le théorème 1.2.2 étend au cas réductible "non dégénéré" (c'est-àdire sous les conditions $C(m+n, m, n))$ les critères d'équivalence du cas irréductible (théorème 1.1.2).

Dans cette section, nous obtenons ainsi un "dictionnaire" entre les conditions paramétriques et les propriétés d'algèbre différentielle dans les cas réductibles.

À la deuxième section, nous calculons le groupe de Galois dans deux cas réductibles "non dégénérés" illustrant bien la méthode algébrique employée ici. Celle-ci permet en fait de traiter tous les opérateurs hypergéométriques ([Bou1] pour le cas général).

Pour simplifier, nous nous limitons au cas confluent où $q \neq p$. Sans perte de généralité, on peut supposer $q>p \geq 0$.

Les groupes de Galois des opérateurs hypergéométriques irréductibles ont été complètement déterminés par F. Beukers, D. Brownawell et G. Heckmann $[\mathrm{BBH}]$, par $\mathrm{O}$. Gabber et N. Katz [Ka] dont nous rappellerons les principaux résultats au paragraphe 2.1.2.

Par des méthodes analytiques, notamment, par le calcul des matrices de Stokes, et sous la seule hypothèse que les paramètres $\mu_{i}$ sont distincts deux à deux modulo $\mathbb{Z}, \mathrm{A}$. Duval et $\mathrm{C}$. Mitschi [DuMi], généralisant des travaux de Ramis [MR], ont déterminé le groupe de Galois de tous les opérateurs hypergéométriques réductibles ou irréductibles avec $q=3$ et 
$p \in\{1,2\}$. C. Mitschi [Mi] a complété cette étude par le calcul d'opérateurs irréductibles d'ordre 4 et dans quelques cas d'ordre quelconque.

Décrivons maintenant la technique employée dans cette deuxième section.

Pour un opérateur $Q$, notons $F_{Q}$ une extension de Picard-Vessiot qui lui est attachée, $G_{Q}$ le $\mathbb{C}$-espace vectoriel de ses solutions et $G_{Q}=$ $\operatorname{Gal}_{\text {diff }}\left(F_{Q} / \mathbb{C}(z)\right)$, son groupe Galois différentiel. Soit l'opérateur hypergéométrique "non dégénéré" :

$$
L=P \mathbf{L} R=\prod_{i \in I} P_{i} \mathbf{L} \prod_{j \in J} P_{j}
$$

avec $P_{i}=\theta+\alpha_{i}-1$ et $\mathbf{L}$, Lie-irréductible (déf. 2.1.3).

On calcule le groupe de Galois de $L$ par des extensions successives. On note $G^{s s}(L)$ l'image de l'homomorphisme canonique de $G_{L}$ dans

$$
\prod_{i \in I \cup J} \operatorname{Aut} V_{P_{i}} \times \text { Aut } V_{\mathbf{L}}
$$

Le groupe $G_{L}$ est ainsi une extension de $G^{s s}(L)$ par le groupe :

$$
G^{u}(L)=\operatorname{Gal}_{\operatorname{diff}}\left(F_{L} / \prod_{i \in I \cup J} F_{P_{i}} \times F_{\mathbf{L}}\right),
$$

lui-même extension du groupe :

$$
K^{u}(L)=\operatorname{Gal}_{\text {diff }}\left(F_{\mathbf{L}} F_{P} F_{R} / \prod_{i \in I \cup J} F_{P_{i}} \times F_{\mathbf{L}}\right),
$$

par le groupe :

$$
H^{u}(L)=\operatorname{Gal}_{\operatorname{diff}}\left(F_{L} / F_{P} F_{\mathbf{L}} F_{R}\right)
$$

On montre que, sous les conditions $C(m+n, m, n)$, ces groupes ne dépendent pas de la décomposition de l'opérateur $L$.

On obtient $G^{s s}(L)$ par des considérations standard sur le compositum d'extensions de Picard-Vessiot.

Le groupe $K^{u}(L)$ est isomorphe à $\mathbb{C}$ si $\log z$ appartient à $F_{P}$ ou à $F_{R}$, c'est-à-dire si l'application $i \mapsto \alpha_{i}$ n'est pas injective sur $I$ ou sur $J$, et, sinon, il est nul.

La partie importante est donc l'étude du groupe $H^{u}(L)$. 
Le premier cas étudié est celui où $r=m$ (ou de façon duale $r=n$ ). Nous déterminons d'abord le groupe $H^{u}(L)=\operatorname{Gal}_{\text {diff }}\left(F_{P \mathbf{L}} / F_{P} F_{\mathbf{L}}\right)$ par les techniques de cohomologie galoisienne de [Be2], inspirées par la méthode de Kummer. Le point crucial est ici que $\mathbf{L}$ étant Lie-irréductible, le groupe $\operatorname{Gal}_{\text {diff }}\left(F_{\mathbf{L}} F_{P} / F_{P}\right)$ opère irréductiblement sur l'espace des solutions de $\mathbf{L}$. Les résultats obtenus pour le calcul de $H^{u}(L)$ resteraient d'ailleurs valables tant que cette condition est réalisée et ce, même si $p=q$.

À cause de l'hypothèse $r=m$, on montre ainsi que le groupe $H^{u}(L)$ est aussi "gros" que possible; par exemple, sous les conditions $C(2,2,0)$ (qui implique en particulier que $L$ est isomorphe à un produit du type $(\theta+\alpha)\left(\theta+\alpha^{\prime}\right) \mathbf{L}=P \mathbf{L}$ avec $\alpha-\alpha^{\prime}$ nul ou non entier $)$, le groupe $H^{u}(L)$ est isomorphe à $\mathbb{C}^{2(q-2)}$.

Le deuxième cas "non dégénéré" étudié est celui où l'opérateur vérifie les conditions $C(2,1,1)$, ce qui implique qu'il est isomorphe à :

$$
L=P \mathbf{L} R=(\theta+\alpha-1) \mathbf{L}\left(\theta+\alpha^{\prime}-1\right) .
$$

On suppose L Lie-irréductible. Le groupe $G_{L}$ est ainsi une extension de $G^{s s}(L)$ par le groupe :

$$
G^{u}(L)=\operatorname{Gal}_{\operatorname{diff}}\left(F_{L} / F_{P} F_{\mathbf{L}} F_{R}\right)
$$

Pour déterminer ce dernier groupe, on applique les résultats précédents aux calculs de $H^{u}(P \mathbf{L})$ et de $H^{u}(\mathbf{L} R)$ et on obtient alors $G^{u}(L)$ grâce aux propriétés que l'on a rassemblé dans l'appendice sur les groupes affines : si les extensions $F_{P \mathbf{L}} F_{R}$ et $F_{\mathbf{L} R} F_{P}$ ont une intersection réduite à $F_{P} F_{R}$, le groupe $G^{u}(L)$ est le plus "gros" possible sinon, il existe $\beta$ tel que l'opérateur "tordu" $L(\theta+\beta)$ est isomorphe à son dual et la dimension de $G^{u}(L)$ est $q-1$ ou $q-2$ selon que $\mathbf{L}(\theta+\beta)$ a un groupe de Galois contenu dans un groupe symplectique ou orthogonal. Par exemple, si le groupe de Galois de L contient le groupe $\mathrm{SL}_{q-2}(\mathbb{C})$, alors $H^{u}(L)$ est isomorphe à $\mathbb{C}^{2 q-3}$.

La section 2 se termine par quelques remarques concernant le cas où l'opérateur vérifie les conditions $C(m+n, m, n)$ avec $m n>1$ et le cas "dégénéré". 


\section{Classification paramétriques des opérateurs hypergéométriques réductibles}

\subsection{Rappels et notations}

On désignera, dans toute la suite, l'ensemble des entiers rationnels par $\mathbb{Z}$, l'ensemble des nombres réels par $\mathbb{R}$, l'ensemble des nombres complexes par $\mathbb{C}$ et le corps $\mathbb{C}(z)$ des fractions rationnelles à coefficients dans $\mathbb{C}$ par $\mathbb{K}$.

\subsubsection{Généralités}

DÉFINITION 1.1.1. - Un corps différentiel est un corps commutatif $K$ muni d'une application $\delta: K \rightarrow K$ vérifiant :

$$
\delta(x+y)=\delta x+\delta y \quad \text { et } \quad \delta(x y)=x \delta y+y \delta x .
$$

On le note $(K, \delta)$.

Si $(K, \delta)$ est un corps différentiel, alors $\{x \in K \mid \delta x=0\}$ est un souscorps de $K$ noté $C_{K}$ et appelé corps des constantes.

DÉFINITION 1.1.2. - On appelle dual (ou adjoint) de l'opérateur $L(\delta)=$ $\sum_{i=0, \ldots, m} a_{i} \delta^{i}$ et l'on note $L^{*}(\delta)$ l'opérateur :

$$
L^{*}(\delta)=\sum_{i=0, \ldots, m}(-1)^{i} \delta^{i} a_{i}
$$

DÉFINITION 1.1.3. - Étant donné un opérateur différentiel $L$ d'ordre $n$, on appelle matrice fondamentale de solutions toute matrice de la forme $\left(f_{k}^{(i)}\right)_{1 \leq k \leq n ; 0 \leq i \leq n-1}$, où $f_{1}, \ldots, f_{n}$ désignent une base de solutions de $L y=0$ dans $F_{L}$ et $f_{k}^{(i)}=\delta^{i} f_{k}$.

Définition 1.1.4. - Soient $M$ et $M^{\prime}$ deux éléments de $K[\delta]$, et $A$ une matrice fondamentale de solutions de l'opérateur $M$. On dira que les opérateurs $M$ et $M^{\prime}$ sont équivalents s'il existe une matrice $\mathrm{N}$ à coefficients dans $K$ telle que $N A$ est une matrice fondamentale de solutions de $M^{\prime}$. 
Proposition 1.1.1. - Les opérateurs $M$ et $M^{\prime}$ de $K[\delta]$ sont équivalents si et seulement s'il existe un opérateur $P$ de $K[\delta]$ tel que l'application : $y \mapsto P y$ est un isomorphisme de l'espace des solutions de $M$ sur celui de $M^{\prime}$.

\subsubsection{Opérateurs hypergéométriques}

Notations. - Soient $q, p$ deux entiers tels que $q \geq p \geq 0$ et $\left\{\mu_{1}, \ldots, \mu_{p}\right\}$, $\left\{\nu_{1}, \ldots, \nu_{q}\right\}$ deux familles de nombres complexes.

On appelle opérateur hypergéométrique associé à ces données l'opérateur différentiel d'ordre $q$ :

$$
L=L\left(\left\{\mu_{i}\right\}_{i},\left\{\nu_{j}\right\}_{j} ; p, q\right)=(-1)^{q-p} z \prod_{i=1, \ldots, p}\left(\theta+\mu_{i}\right)-\prod_{j=1, \ldots, q}\left(\theta+\nu_{j}-1\right)
$$

où $\theta=z(\mathrm{~d} / \mathrm{d} z)$.

On posera pour ces mêmes données :

$$
L^{\prime}\left(\left\{\mu_{i}\right\}_{i},\left\{\nu_{j}\right\}_{j} ; p, q\right)=(-1)^{q}\left\{z \prod_{i=1, \ldots, p}\left(\theta+\mu_{i}\right)-\prod_{j=1, \ldots, q}\left(\theta+\nu_{j}-1\right)\right\} .
$$

Remarque 1.1.1. - Si $p-q$ est pair, les opérateurs

$$
L^{\prime}\left(\left\{\mu_{i}\right\}_{i},\left\{\nu_{j}\right\}_{j} ; p, q\right) \text { et } L\left(\left\{\mu_{i}\right\}_{i},\left\{\nu_{j}\right\}_{j} ; p, q\right)
$$

désignent le même opérateur au signe près et si $p-q$ est impair on passe de l'un à l'autre, au signe près, par le changement de variable $z$ en $-z$.

Nous reproduisons maintenant les résultats de $[\mathrm{BBH}]$ et [Ka] qui seront nécessaires pour la suite.

LEMME 1.1.1 ([Ka, sect. 3.1]). - Le dual de l'opérateur

$$
L\left(\left\{\mu_{i}\right\}_{i},\left\{\nu_{j}\right\}_{j} ; p, q\right)
$$

par rapport à la dérivation $\theta$ est l'opérateur :

$$
L^{\prime}\left(\left\{-\mu_{i}+1\right\}_{i},\left\{-\nu_{j}+2\right\}_{j} ; p, q\right) .
$$


THÉORÈme 1.1.1 ([BBH, lemme 4.2] ou [Ka]). - L'opérateur $L$ (ou $L^{\prime}$ ) est irréductible sur $\mathbb{K}$ si et seulement si pour tout couple $\{i, j\}$ d'indices, le nombre $\mu_{i}-\nu_{j}$ n'est pas un entier rationnel.

THÉORÈME 1.1.2 ([Ka]). - Si l'opérateur L est irréductible, sa classe d'isomorphisme ne dépend que de la classe modulo $\mathbb{Z}$ de ses paramètres.

\subsection{Décompositions d'un opérateur et conditions $C(r, m, n)$}

\subsubsection{Définitions et énoncé des résultats}

Soit

$$
L=L\left(\left\{\mu_{i}\right\}_{i},\left\{\nu_{j}\right\}_{j} ; p, q\right)=(-1)^{q-p} z \prod_{i=1, \ldots, p}\left(\theta+\mu_{i}\right)-\prod_{j=1, \ldots, q}\left(\theta+\nu_{j}-1\right)
$$

un opérateur hypergéométrique généralisé.

DÉFInITION 1.2.1. - Soient $P$ et $Q$ deux opérateurs différentiels, on dit que $P$ divise $Q$ à gauche (resp. à droite), à isomorphisme près, si $P$ divise à gauche (resp. à droite) un opérateur équivalent à $Q$.

Remarque 1.2.1. - $A$ un opérateur différentiel $L \in K[\theta]$, on peut associer un $D$-module ou un sous-module sur l'anneau $K[\theta]: K[\theta] / K[\theta] L([\mathrm{Be} 1]$, [De], [Ka1]). Si l'opérateur $L$ se décompose en produits d'opérateurs irréductibles; $\prod L_{i}$, on obtient une suite de Jordan-Hölder dont les quotients sont isomorphes aux $D$-modules associés aux opérateurs irréductibles $K[\theta] / K[\theta] L_{i}$. Deux suites de Jordan-Hölder étant isomorphes [Bo], on en déduit que la collection des classes d'équivalences des opérateurs irréductibles entrant dans la décomposition d'un opérateur donné est unique mais il n'y a pas unicité des facteurs (voir Exemple).

DÉFINITION 1.2.2. - Soit l'opérateur hypergéométrique :

$$
L=L\left(\left\{\mu_{i}\right\}_{i},\left\{\nu_{j}\right\}_{j} ; p, q\right) .
$$

On désigne par $r_{L}, m_{L}, n_{L}, r_{L}^{\prime}, m_{L}^{\prime}, n_{L}^{\prime}, n_{L}^{\prime \prime}$, les entiers tels que :

- l'opérateur $L$ est produit de $r_{L}+1$ opérateurs irréductibles;

- $m_{L}$ est le plus grand des nombres entiers vérifiant : il existe $m_{L}$ éléments $\alpha_{i}$ de $\mathbb{C}$ tels que $\prod_{i \in\{1, \ldots, n\}}\left(\theta+\alpha_{i}\right)$ divise l'opérateur $L \grave{a}$ gauche, à isomorphisme près; 
- $n_{L}$ est le plus grand des nombres entiers vérifiant : il existe $n_{L}$ éléments $\alpha_{i}$ de $\mathbb{C}$ tels que $\prod_{i \in\{1, \ldots, n\}}\left(\theta+\alpha_{i}\right)$ divise l'opérateur $L$ à droite, à isomorphisme près;

- $r_{L}^{\prime}$ est le plus grand des nombres entiers vérifiant : il existe $r_{L}^{\prime}$ indices distincts $i_{k}$ dans $\{1, \ldots, p\}$ et $r_{L}^{\prime}$ indices distincts $j_{k}$ dans $\{1, \ldots, q\}$ tels que $\mu_{i_{k}}-\nu_{j_{k}}$ est un entier;

- $m_{L}^{\prime}$ est le plus grand des nombres entiers vérifiant : il existe $m_{L}^{\prime}$ indices distincts $i_{k}$ dans $\{1, \ldots, p\}$ et $m_{L}^{\prime}$ indices distincts $j_{k}$ dans $\{1, \ldots, q\}$ tels que $\mu_{i_{k}}-\nu_{j_{k}}$ est un entier positif ou nul;

- $n_{L}^{\prime}$ est le plus grand des nombres entiers vérifiant : il existe $n_{L}^{\prime}$ indices distincts $i_{k}$ dans $\{1, \ldots, p\}$ et $n_{L}^{\prime}$ indices distincts $j_{k}$ dans $\{1, \ldots, q\}$ tels que $\mu_{i_{k}}-\nu_{j_{k}}$ est un entier strictement négatif.

- $n_{L}^{\prime \prime}$ est le nombre exact de solutions linéairement indépendantes de l'opérateur $L$ dans la $\mathbb{K}$-algèbre engendrée par $\log z$ et les $z^{\alpha_{i}}$ où les $\alpha_{i}$ parcourent $\mathbb{C}$.

On suppose désormais $q-r_{L}^{\prime} \geq 1$. Dans le cas contraire, on adapte facilement les résultats qui suivent à ce cas dégénéré.

D'après le théorème 1.1.1, l'opérateur hypergéométrique :

$$
L\left(\left\{\mu_{i}\right\}_{i},\left\{\nu_{j}\right\}_{j} ; p, q\right)
$$

est irréductible si et seulement si, pour tout couple $(i, j)$ d'indices, $\mu_{i}-\nu_{j}$ n'est pas entier. Dans le cas où il est réductible, nous obtenons le résultat suivant.

THÉORÈME 1.2.1.- Soit l'opérateur hypergéométrique :

$$
L=L\left(\left\{\mu_{i}\right\}_{i},\left\{\nu_{j}\right\}_{j} ; p, q\right) \text {. }
$$

On $a$ :

$$
r_{L}=r_{L}^{\prime}, \quad m_{L}=m_{L}^{\prime}, \quad n_{L}=n_{L}^{\prime}=n_{L}^{\prime \prime}
$$

Ceci conduit à poser les définitions suivantes.

DÉfINITION 1.2.3. - Étant donnés les entiers positifs $r, m, n$, on dit que $L$ vérifie les conditions $C(r, m, n)$ si et seulement si :

$$
r=r_{L}, \quad m=m_{L}, \quad n=n_{L} .
$$


Remarque. - Soit $L$ un opérateur hypergéométrique. On a évidemment les relations :

$$
m_{L} \leq r_{L}, \quad n_{L} \leq r_{L}, \quad m_{L}+n_{L} \geq r_{L}
$$

Exemple. - Considérons les opérateurs :

$$
L=L(\{0,0, \alpha\},\{0,1, \beta, \gamma, \delta\} ; 3,5)
$$

et

$$
L^{\prime}=L(\{-1,0, \alpha\},\{0,1, \beta, \gamma, \delta\} ; 3,5)
$$

où $\alpha, \beta, \gamma$ et $\delta$ sont des nombres complexes non entiers et distincts deux à deux modulo $\mathbb{Z}$.

L'opérateur $L$ vérifie les conditions $C(2,1,1)$ et admet la décomposition :

$$
L=(\theta-1) L(\{\alpha\},\{\beta, \gamma, \delta\} ; 1,3) \theta .
$$

L'opérateur $L^{\prime}$ vérifie les conditions $C(2,1,2)$ et admet les décompositions :

$$
L^{\prime}=L(\{\alpha\},\{\beta, \gamma, \delta\} ; 1,3) \theta(\theta-1)
$$

et

$$
L^{\prime}=(\theta-1) L(\{-1, \alpha\},\{1, \beta, \gamma, \delta\} ; 2,4)
$$

La démonstration du théorème 1.2.1 fait l'objet des deux paragraphes suivants 1.2 .2 et 1.2 .3 .

\subsubsection{Lemmes préliminaires}

LEMME 1.2.1

i) L'opérateur hypergéométrique

$$
L=L\left(\left\{\mu_{i}\right\}_{i},\left\{\nu_{j}\right\}_{j} ; p, q\right)
$$

est divisible à droite par $\theta+\alpha$ si et seulement s'il existe $(i, j)$ tel que $\left(\mu_{i}, \nu_{j}\right)=(\alpha, \alpha+1)$.

ii) L'opérateur $L$ est divisible à gauche par $\theta+\alpha$ si et seulement s'il existe $(i, j)$ tel que $\left(\mu_{i}, \nu_{j}\right)=(\alpha+1, \alpha+1)$. 
Démonstration. - S'il existe $(i, j)$ tel que $\left(\mu_{i}, \nu_{j}\right)=(\alpha, \alpha+1)$, l'opérateur $L$ est clairement divisible à droite par $\theta+\alpha$.

Supposons que l'opérateur $L$ est divisible à droite par $\theta+\alpha$. Cela signifie que $z^{-\alpha}$ est une solution de $L$.

Comme pour tout $\beta$ de $\mathbb{C},(\theta+\beta)\left(z^{-\alpha}\right)=(-\alpha+\beta)\left(z^{-\alpha}\right)$, on a :

$$
\begin{aligned}
& L\left(z^{-\alpha}\right)= \\
& =\left\{(-1)^{q-p} z \prod_{i=1, \ldots, p}\left(-\alpha+\mu_{i}\right)-\prod_{j=1, \ldots, q}\left(-\alpha+\nu_{j}-1\right)\right\}\left(z^{-\alpha}\right)=0 .
\end{aligned}
$$

D'où pour tout $z$ de $\mathbb{C}$ :

$$
(-1)^{q-p} z \prod_{i=1, \ldots, p}\left(-\alpha+\mu_{i}\right)-\prod_{j=1, \ldots, q}\left(-\alpha+\nu_{j}-1\right)=0 .
$$

En prenant $z=0$, on obtient $\prod_{j=1, \ldots, q}\left(-\alpha+\nu_{j}-1\right)=0$, puis $\prod_{i=1, \ldots, p}\left(-\alpha+\mu_{i}\right)=0$. Il existe donc $i$ et $j$ tels que $-\alpha+\nu_{j}-1=0$ et $-\alpha+\mu_{i}=0$, donc tels que $\left(\mu_{i}, \nu_{j}\right)=(\alpha, \alpha+1)$.

On déduit ii) par dualité d'après le lemme 1.1.1 et la remarque 1.1.1 qui le précède.

Notation. - Étant donnés les nombres complexes $\alpha$ et $\beta$, on note $[[\alpha, \beta]]$ :

$$
\{\lambda \in \mathbb{C}, \lambda-\alpha \in \mathbb{Z}, \lambda-\beta \in \mathbb{Z} \text { et } \Re \lambda \in[\Re \alpha, \Re \beta]\} \text {. }
$$

On définit de manière analogue $]] \alpha, \beta]],[[\alpha, \beta[[$ et $]] \alpha, \beta[[$.

LEMME 1.2.2. - La classe d'équivalence d'un opérateur hypergéométrique reste inchangée sous les opérations suivantes :

1) en remplaçant $\mu_{k}$ par $\mu_{k}+1$ si pour tout $j, \nu_{j} \neq \mu_{k}+1$;

2) en remplaçant $\mu_{k}$ par $\mu_{k}-1$ si pour tout $j, \nu_{j} \neq \mu_{k}$;

3) en remplaçant $\nu_{k}$ par $\nu_{k}+1$ si pour tout $i, \mu_{i} \neq \nu_{k}$;

4) en remplaçant $\nu_{k}$ par $\nu_{k}-1$ si pour tout $i, \mu_{i} \neq \nu_{k}-1$;

5) en remplaçant $\mu_{k}$ par $\mu_{k}+t$, où $t$ est un entier $\geq 0$, si pour tout $j$, $\left.\left.\left.\left.\nu_{j} \notin\right]\right] \mu_{k}, \mu_{k}+t\right]\right]$;

6) en remplaçant $\nu_{k}$ par $\nu_{k}+t$, où $t$ est un entier $\geq 0$, si pour tout $i$, $\mu_{i} \notin\left[\left[\nu_{k}, \nu_{k}+t[[\right.\right.$. 


\section{Démonstration}

1) (Voir aussi [Ro, lemme 2.2] et [BH, p. 329]) De la relation : $\left(\theta+\mu_{k}\right) z=$ $z\left(\theta+\mu_{k}+1\right)$, on déduit :

$$
\begin{aligned}
& \left(\theta+\mu_{k}\right) L= \\
& =\left(\theta+\mu_{k}\right)\left\{(-1)^{q-p} z \prod_{i=1, \ldots, p}\left(\theta+\mu_{i}\right)-\prod_{j=1, \ldots, q}\left(\theta+\nu_{j}-1\right)\right\}
\end{aligned}
$$

soit :

$$
\begin{aligned}
& \left(\theta+\mu_{k}\right) L= \\
& =\left\{(-1)^{q-p} z\left(\theta+\mu_{k}+1\right) \prod_{i \neq k}\left(\theta+\mu_{i}\right)-\prod_{j=1, \ldots, q}\left(\theta+\nu_{j}-1\right)\right\}\left(\theta+\mu_{k}\right) \\
& =L^{\prime}\left(\theta+\mu_{k}\right) .
\end{aligned}
$$

Il suffit de montrer que l'application linéaire qui, à $Y$, associe $\left(\theta+\mu_{k}\right) Y$ envoie de façon injective les solutions de $L$ sur les solutions de $L^{\prime}$.

Il s'agit donc de voir que $\theta+\mu_{k}$ n'annule aucune solution de $L$. Si c'était le cas, $L$ serait divisible à droite $\operatorname{par} \theta+\mu_{k}$. Or, $\theta+\mu_{k}$ divise $\prod_{i=1, \ldots, p}\left(\theta+\mu_{i}\right)$, donc $\theta+\mu_{k}$ diviserait $\prod_{j=1, \ldots, q}\left(\theta+\nu_{j}-1\right)$, ce qui est contraire à notre hypothèse qui entraîne que, pour tout $j$, on a :

$$
\nu_{j} \neq \mu_{k}+1 \text {. }
$$

Donc $\theta+\mu_{k}$ ne divise pas à droite $L$. Les opérateurs $L$ et $L^{\prime}$ sont bien équivalents.

2) Il suffit d'appliquer 1).

5) Il suffit d'appliquer 1) successivement à $\mu_{k}+1, \ldots \mu_{k}+t-1$.

6) Remarquons que la démonstration n'a pas fait intervenir la relation $p<q$ et que le résultat est valable pour les opérateurs hypergéométriques :

$$
L^{\prime}\left(\left\{\mu_{i}\right\}_{i},\left\{\mu_{j}\right\}_{j} ; p, q\right)
$$

d'après la remarque 1.1.1. Après le changement de variable $z$ en $z^{\prime}=1 / z$, si $\theta^{\prime}=z^{\prime}\left(\mathrm{d} / \mathrm{d} z^{\prime}\right)$, l'opérateur devient :

$$
L=(-1)^{q-p} \prod_{i=1, \ldots, p}\left(-\theta^{\prime}+\mu_{i}\right)-z^{\prime} \prod_{j=1, \ldots, q}\left(-\theta^{\prime}+\nu_{j}-1\right)
$$


soit

$$
L=(-1)^{q}\left\{\prod_{i=1, \ldots, p}\left(\theta^{\prime}-\mu_{i}\right)-z^{\prime} \prod_{j=1, \ldots, q}\left(\theta^{\prime}-\nu_{j}+1\right)\right\}
$$

soit

$$
L=L^{\prime}\left(\left\{-\nu_{j}+1\right\}_{j},\left\{-\mu_{i}+1\right\}_{i} ; q, p\right)
$$

relativement à l'opérateur $\theta^{\prime}$.

D'après 5), si pour tout $i$, on a

$$
-\mu_{i}+1 \notin\left\{-\nu_{k}-t+2, \ldots,-\nu_{k}+1\right\},
$$

c'est-à-dire, si pour tout $i$, on a $\mu_{i} \notin\left\{\nu_{k}, \ldots, \nu_{k}+t-1\right\}$, alors les opérateurs :

$$
L^{\prime}\left(\left\{-\nu_{j}+1\right\}_{j},\left\{-\mu_{i}+1\right\}_{i} ; q, p\right)
$$

et

$$
L^{\prime}\left(\left\{\left\{-\nu_{k}-t+1\right\},\left\{-\nu_{j}+1\right\}_{j \neq k}\right\},\left\{-\mu_{i}+1\right\}_{i} ; q, p\right)
$$

sont équivalents.

3) et 4) Il suffit d'appliquer 6).

LEMME 1.2.3. - Soient les opérateurs :

$$
L_{1}=(\theta+\alpha-1)^{p^{\prime}}(\theta+\alpha)^{p^{\prime \prime}} L\left(\left\{\mu_{i}\right\}_{i \in I^{*}},\left\{\nu_{j}\right\}_{j \in J^{*}} ; p_{1}, q_{1}\right)
$$

et

$$
L_{2}=(\theta+\alpha-1)^{p^{\prime \prime}}(\theta+\alpha)^{p^{\prime}} L\left(\left\{\mu_{i}\right\}_{i \in I^{*}},\left\{\nu_{j}\right\}_{j \in J^{*}} ; p_{1}, q_{1}\right)
$$

Si pour tout $i \in I^{*}, \mu_{i} \neq \alpha$ et, pour tout $j \in J^{*}, \nu_{j} \neq \alpha+1$ alors les opérateurs $L_{1}$ et $L_{2}$ sont équivalents. Autrement dit, soit l'opérateur:

$$
\begin{aligned}
L=L\left(\left\{\mu_{i}, \alpha, \ldots, \alpha, \alpha+1, \ldots, \alpha+1\right\}\right. & \\
& \left.\left\{\nu_{j}, \alpha, \ldots, \alpha, \alpha+1, \ldots, \alpha+1\right\} ; p, q\right)
\end{aligned}
$$

avec $\alpha$ répété $p^{\prime}$ fois et $\alpha+1$ répété $p^{\prime \prime}$ fois.

Si, pour tout $i, \mu_{i} \neq \alpha$ et, pour tout $j, \nu_{j} \neq \alpha+1$ alors on obtient un opérateur équivalent en répétant $\alpha$ et $\alpha+1$ respectivement $p^{\prime \prime}$ et $p^{\prime}$ fois. 
Démonstration. - On peut supposer pour fixer les idées que $p^{\prime \prime} \geq p^{\prime}$.

L'opérateur $L_{1}$ est clairement divisible à droite par $(\theta+\alpha)^{p^{\prime}}$. Il admet donc, comme solutions, les solutions de $(\theta+\alpha)^{p^{\prime}}$ et de

$$
L_{1}^{\prime}=(\theta+\alpha)^{p^{\prime \prime}} L\left(\left\{\mu_{i}\right\}_{i \in I^{*}},\left\{\nu_{j}\right\}_{j \in J^{*}} ; p_{1}, q_{1}\right) \text {. }
$$

Comme on a

$$
L_{1}^{\prime}=(-1)^{q-p} z(\theta+\alpha+1)^{p^{\prime \prime}} \prod_{i \in I^{*}}\left(\theta+\mu_{i}\right)-(\theta+\alpha)^{p^{\prime \prime}} \prod_{j \in J^{*}}\left(\theta+\nu_{j}-1\right)
$$

et que, pour tout $i \in I^{*}, \mu_{i} \neq \alpha$, l'opérateur $L_{1}^{\prime}$ n'est pas divisible à droite $\operatorname{par} \theta+\alpha$. On en déduit que la réunion d'une base de solutions de $(\theta+\alpha)^{p^{\prime}}$ et d'une base de solutions de $L_{1}^{\prime}$ forme une base de solutions de $L_{1}$.

De même, l'opérateur $L_{2}$ est clairement divisible à droite par $(\theta+\alpha)^{p^{\prime}}$ et admet, comme solutions, les solutions de $(\theta+\alpha)^{p^{\prime}}$ et de

$$
L_{2}^{\prime}=(\theta+\alpha-1)^{p^{\prime \prime}} L\left(\left\{\mu_{i}\right\}_{i \in I^{*}},\left\{\nu_{j}\right\}_{j \in J^{*}} ; p_{1}, q_{1}\right) .
$$

Comme on a

$$
L_{2}^{\prime}=(-1)^{q-p} z(\theta+\alpha)^{p^{\prime \prime}} \prod_{i \in I^{*}}\left(\theta+\mu_{i}\right)-(\theta+\alpha-1)^{p^{\prime \prime}} \prod_{j \in J^{*}}\left(\theta+\nu_{j}-1\right)
$$

et que, pour tout $j \in J^{*}, \nu_{j} \neq \alpha+1$, l'opérateur $L_{2}^{\prime}$ n'est pas divisible à droite $\operatorname{par} \theta+\alpha$. On en déduit que la réunion d'une base de solutions de $(\theta+\alpha)^{p^{\prime}}$ et d'une base de solutions de $L_{2}^{\prime}$ forme une base de solutions de $L_{2}$.

D'après le lemme 1.2.2, les opérateurs $L_{1}^{\prime}$ et $L_{2}^{\prime}$ sont équivalents. On en conclut que les opérateurs $L_{1}$ et $L_{2}$ sont équivalents.

Lemme 1.2.4. - Soient $p^{\prime}$ et $p^{\prime \prime}$ deux entiers $>0$ tels que $p^{\prime \prime} \geq p^{\prime}$ et soient les opérateurs :

$$
L_{1}=(\theta+\alpha-1)^{p^{\prime}}(\theta+\alpha)^{p^{\prime \prime}} L\left(\left\{\mu_{i}\right\}_{i \in I^{*}},\left\{\alpha+1,\left\{\nu_{j}\right\}_{j \in J^{*}}\right\} ; p_{1}, q_{1}\right)
$$

et

$$
L_{2}=(\theta+\alpha-1)^{p^{\prime}}(\theta+\alpha)^{p^{\prime \prime}} L\left(\left\{\mu_{i}\right\}_{i \in I^{*}},\left\{\alpha,\left\{\nu_{j}\right\}_{j \in J^{*}}\right\} ; p_{1}, q_{1}\right)
$$


Si pour tout $i \in I^{*}, \mu_{i} \neq \alpha$, alors les opérateurs $L_{1}$ et $L_{2}$ sont équivalents. Autrement dit, soit l'opérateur:

$$
\begin{aligned}
L=L\left(\left\{\mu_{i}, \alpha, \ldots, \alpha, \alpha+1, \ldots, \alpha+1\right\},\right. & \\
& \left.\left\{\nu_{j}, \alpha, \ldots, \alpha, \alpha+1, \ldots, \alpha+1\right\} ; p, q\right)
\end{aligned}
$$

avec $\alpha$ répété $p^{\prime}$ fois, $\alpha+1$ répété $p^{\prime \prime}$ fois comme paramètre $\mu$ et $p^{\prime \prime}+1$ comme paramètre $\nu$.

Si $p^{\prime \prime} \geq p^{\prime}$ et si, pour tout $i, \mu_{i} \neq \alpha$, alors on obtient un opérateur équivalent à $L$ en remplaçant un paramètre $\nu$ égal à $\alpha+1$ par $\alpha$.

Démonstration. - L'opérateur $L_{1}$ est clairement divisible à droite par $(\theta+\alpha)^{p^{\prime}}$. Il admet donc, comme solutions, les solutions de $(\theta+\alpha)^{p^{\prime}}$ et de

$$
L_{1}^{\prime}=(\theta+\alpha)^{p^{\prime \prime}} L\left(\left\{\mu_{i}\right\}_{i \in I^{*}},\left\{\alpha+1,\left\{\nu_{j}\right\}_{j \in J^{*}}\right\} ; p_{1}, q_{1}\right) .
$$

Comme on a :

$$
L_{1}^{\prime}=(-1)^{q-p} z(\theta+\alpha+1)^{p^{\prime \prime}} \prod_{i \in I^{*}}\left(\theta+\mu_{i}\right)-(\theta+\alpha)^{p^{\prime \prime}+1} \prod_{j \in J^{*}}\left(\theta+\nu_{j}-1\right)
$$

et que, pour tout $i \in I^{*}, \mu_{i} \neq \alpha$, l'opérateur $L_{1}^{\prime}$ n'est pas divisible à droite $\operatorname{par} \theta+\alpha$. On en déduit que la réunion d'une base de solutions de $(\theta+\alpha)^{p^{\prime}}$ et d'une base de solutions de $L_{1}^{\prime}$ forme une base de solutions de $L_{1}$.

De même, l'opérateur $L_{2}$ est clairement divisible à droite par $(\theta+\alpha)^{p^{\prime}}$ et admet, comme solutions, les solutions de $(\theta+\alpha)^{p^{\prime}}$ et de

$$
L_{2}^{\prime}=(\theta+\alpha)^{p^{\prime \prime}} L\left(\left\{\mu_{i}\right\}_{i \in I^{*}},\left\{\alpha,\left\{\nu_{j}\right\}_{j \in J^{*}}\right\} ; p_{1}, q_{1}\right)
$$

Comme on a :

$$
L_{2}^{\prime}=(-1)^{q-p} z(\theta+\alpha+1)^{p^{\prime \prime}} \prod_{i \in I^{*}}\left(\theta+\mu_{i}\right)-(\theta+\alpha-1)(\theta+\alpha)^{p^{\prime \prime}} \prod_{j \in J^{*}}\left(\theta+\nu_{j}-1\right)
$$

et que, pour tout $i \in I^{*}, \mu_{i} \neq \alpha$, l'opérateur $L_{2}^{\prime}$ n'est pas divisible à droite par $\theta+\alpha$. On en déduit que la réunion d'une base de solutions de $(\theta+\alpha)^{p^{\prime}}$ et d'une base de solutions de $L_{2}^{\prime}$ forme une base de solutions de $L_{2}$.

D'après le lemme 1.2.2, les opérateurs $L_{1}^{\prime}$ et $L_{2}^{\prime}$ sont équivalents. On en conclut que les opérateurs $L_{1}$ et $L_{2}$ sont équivalents. 
LEMME 1.2.5. - Soit s un entier $>0$ et $\left\{p_{1}, \ldots, p_{s}\right\}$ une suite décroissante d'entiers $>0$. Considérons les opérateurs

$$
\begin{aligned}
L_{1}= & \prod_{t \in\{1, \ldots, s\}}(\theta+\alpha-t)^{p_{t}} L\left(\left\{\alpha,\left\{\mu_{i}\right\}_{i \in I^{\prime}}\right\},\left\{\alpha-s+1,\left\{\nu_{j}\right\}_{j \in J^{\prime}}\right\} ; p^{\prime}, q^{\prime}\right) \\
= & \prod_{t \in\{1, \ldots, s\}}(\theta+\alpha-t)^{p_{t}} \times \\
& \times\left\{(-1)^{q^{\prime}-p^{\prime}} z(\theta+\alpha) \prod_{i \in I^{\prime}}\left(\theta+\mu_{i}\right)-(\theta+\alpha-s) \prod_{j \in J^{\prime}}\left(\theta+\nu_{j}-1\right)\right\}
\end{aligned}
$$

et

$$
\begin{aligned}
L_{2}= & (\theta+\alpha-1)^{p_{1}+1} \prod_{t \in\{2, \ldots, s\}}(\theta+\alpha-t)^{p_{t}} \times \\
& \times L\left(\left\{\mu_{i}\right\}_{i \in I^{\prime}},\left\{\nu_{j}\right\}_{j \in J^{\prime}} ; p^{\prime}-1, q^{\prime}-1\right) \\
= & (\theta+\alpha-1)^{p_{1}+1} \prod_{t \in\{2, \ldots, s\}}(\theta+\alpha-t)^{p_{t}} \times \\
& \times\left\{(-1)^{q^{\prime}-p^{\prime}} z \prod_{i \in I^{\prime}}\left(\theta+\mu_{i}\right)-\prod_{j \in J^{\prime}}\left(\theta+\nu_{j}-1\right)\right\} .
\end{aligned}
$$

Si, pour tout $i \in I^{\prime}, \mu_{i} \notin\{\alpha-s+1, \ldots, \alpha-1\}$, alors les opérateurs $L_{1}$ et $L_{2}$ sont équivalents.

Démonstration. - L'opérateur $L_{1}$ est clairement divisible à droite par

$$
\prod_{t \in\{2, \ldots, s\}}(\theta+\alpha-t+1)^{p_{t}}
$$

Donc, les solutions de $\prod_{t \in\{2, \ldots, s\}}(\theta+\alpha-t+1)^{p_{t}}$ et de

$$
L_{1}^{\prime}=(\theta+\alpha-1)^{p_{1}} L\left(\left\{\alpha,\left\{\mu_{i}\right\}_{i \in I^{\prime}}\right\},\left\{\alpha-s+1,\left\{\nu_{j}\right\}_{j \in J^{\prime}}\right\} ; p^{\prime}, q^{\prime}\right)
$$

sont des solutions de $L_{1}$. Comme pour tout $i \in I^{\prime}, \mu_{i} \notin\{\alpha-s+1, \ldots, \alpha-1\}$, alors, d'après le lemme 1.2.1, l'opérateur $L_{1}^{\prime}$ n'est pas divisible par $\theta+\alpha-$ $t+1, t \in\{2, \ldots, s\}$. On en déduit que la réunion d'une base de solutions de $\prod_{t \in\{2, \ldots, s\}}(\theta+\alpha-t+1)^{p_{t}}$ et d'une base de solutions de $L_{1}^{\prime}$ forme une base de solutions de $L_{1}$. 
De même l'opérateur $L_{2}$ est divisible à droite par $\prod_{t \in\{2, \ldots, s\}}(\theta+\alpha-$ $t+1)^{p_{t}}$. Comme précédemment, on montre que la réunion d'une base de solutions de $\prod_{t \in\{2, \ldots, s\}}(\theta+\alpha-t+1)^{p_{t}}$ et d'une base de solutions de

$$
L_{2}^{\prime}=(\theta+\alpha-1)^{p_{1}+1}\left\{(-1)^{q^{\prime}-p^{\prime}} z \prod_{i \in I^{\prime}}\left(\theta+\mu_{i}\right)-\prod_{j \in J^{\prime}}\left(\theta+\nu_{j}-1\right)\right\}
$$

forme une base de solutions de $L_{2}$.

D'après le lemme 1.2.2, on obtient un opérateur équivalent à $L_{1}^{\prime}$ en remplaçant un paramètre $\nu$ égal à $\alpha-s+1$ par $\alpha$. Les opérateurs $L_{1}^{\prime}$ et $L_{2}^{\prime}$ sont donc équivalents. Il en résulte que $L_{1}$ et $L_{2}$ le sont aussi.

Rappel.- On appelle fonction hypergéométrique une fonction de la forme :

$$
{ }_{p} F_{q}(\{\mu\},\{\nu\} \mid z)=\sum_{n=0}^{\infty} \frac{\left(\mu_{1}\right)_{n} \ldots\left(\mu_{p}\right)_{n}}{\left(\nu_{1}\right)_{n} \ldots\left(\nu_{q}\right)_{n}} \frac{z^{n}}{n !}
$$

avec pour tout $j, \nu_{j} \notin\{0,-1,-2, \ldots\}$, et où $(\alpha)_{n}=\alpha(\alpha+1) \ldots(\alpha+n-1)$ et $(\alpha)_{0}=1$.

Lemme 1.2.6. - Soit $\left.L=L\left(\left\{\mu_{i}\right\},\left\{\nu_{j}\right\}\right) ; p, q\right)$. Si L est divisible à droite par un opérateur $L_{1}$ à coefficients dans $\mathbb{K}$, alors $L_{1}$ a une solution de la forme :

$$
z^{1-\nu_{t}}\left(P(z)+C_{p} F_{q}\left(\left\{\mu_{j}+1-\nu_{k}\right\},\left\{\nu_{j}+1-\nu_{k}\right\} \mid-z\right)\right)
$$

avec pour tout $j, \nu_{j}+1-\nu_{k} \notin\{0,-1,-2, \ldots\}, C$ constante, $\nu_{t}-\nu_{k}$ un entier $\geq 0$ et $P$ un polynôme.

Démonstration. - Soit $V_{1}$ l'espace des solutions de $L_{1}$ et soit $f \in V_{1}$ un vecteur propre sous l'action de la monodromie en 0 (comme $V_{1}$ est stable sous l'action de la monodromie en 0 , un tel vecteur propre existe nécessairement). Alors $f(z)=z^{\alpha} g(z)$ où $\alpha$ est un nombre complexe et $g$ une fonction holomorphe en 0 avec $g(0)=1$. Puisque $f$ est aussi une solution de $L$, on a que $\alpha$ est une solution de l'équation indicielle en 0 de $L$ et par conséquent, il existe $t$ tel que $\alpha=1-\nu_{t}$.

Soit $\nu_{k}$ un des paramètres de $L$ de plus petite partie réelle parmi les paramètres $\nu_{j}$ congrus à $\nu_{t}$ modulo $\mathbb{Z}$. Posons $m=\nu_{t}-\nu_{k}$. On a $m \in \mathbb{Z}$, $m \geq 0$. 
La fonction hypergéométrique

$$
F={ }_{p} F_{q}\left(\left\{\mu_{j}+1-\nu_{k}\right\},\left\{\nu_{j}+1-\nu_{k}\right\} \mid-z\right)
$$

est bien définie. Posons

$$
g(z)=\sum_{n=0}^{\infty} a_{n} z^{n}
$$

et

$$
{ }_{p} F_{q}\left(\left\{\mu_{j}+1-\nu_{k}\right\},\left\{\nu_{j}+1-\nu_{k}\right\} \mid-z\right)=\sum_{n=0}^{\infty} b_{n} z^{n}
$$

Comme $f$ est solution de $L$, on obtient pour $s \geq 1$ :

$$
(-1)^{q-p} \prod_{j}\left(s+\mu_{j}-\nu_{t}\right)(-1)^{p} a_{s-1}-\prod_{j}\left(s+\nu_{j}-\nu_{t}\right)(-1)^{q} a_{s}=0 .
$$

De même pour $s \geq 1$, on a :

$$
(-1)^{q-p} \prod_{j}\left(s+\mu_{j}-\nu_{k}\right)(-1)^{p} b_{s-1}-\prod_{j}\left(s+\nu_{j}-\nu_{k}\right)(-1)^{q} b_{s}=0 .
$$

Dès que $s \geq m+1, s+\nu_{j}-\nu_{t}>0$ pour $\nu_{j}-\nu_{t} \in \mathbb{Z}$ et les suites $\left(a_{s+m}\right)_{s \geq 0}$ et $\left(b_{s}\right)_{s \geq 0}$ vérifient la même relation de récurrence d'ordre un. Elles sont donc proportionnelles.

Si $\left(b_{s}\right)_{s>0}$ n'a aucun de ses termes nul, il existe donc une constante $C$ telle que la fonction $g-C F$ soit égale à un polynôme.

Supposons maintenant qu'il existe un entier $n^{\prime}$ (choisi minimal) tel que $b_{n^{\prime}}=0$ (comme $b_{0}=1, n^{\prime} \geq 1$ ). Alors d'après (2) on a, pour tout $n \geq n^{\prime}$, $b_{n}=0$ et le coefficient de $b_{n^{\prime}-1}$ est nul. Par conséquent, d'après (1), $a_{n^{\prime}+m}$ est nul ainsi que les $a_{s}$ suivants. Dans ce cas $g$ et $F$ sont des polynômes.

Dans les deux cas, on obtient ainsi l'énoncé du lemme.

Remarque. - La preuve ci-dessus s'inspire de la démonstration du lemme 4.2 de $[\mathrm{BBH}]$, modifiée pour tenir compte des cas de dégénérescence de $F$. Nous remercions le referee de nous avoir signalé ce type d'énoncé. 
1.2.3 Décomposition d'un opérateur et conditions sur les paramètres

\section{Proposition 1.2.1}

i) Si l'opérateur hypergéométrique $L$ possède des paramètres tels que $\mu_{i_{1}}-\nu_{j_{1}}$ est un entier $\geq 0$, et si l'on choisit les indices de telle sorte que :

$$
\Re \nu_{j_{1}}=\sup \left\{\Re \nu_{j} \mid \mu_{i_{1}}-\nu_{j} \in \mathbb{Z}, \mu_{i_{1}}-\nu_{j} \geq 0\right\}
$$

alors l'opérateur L est équivalent à

$$
L^{\prime}=\left(\theta+\nu_{j_{1}}-1\right) L\left(\left\{\mu_{i}\right\}_{i \neq i_{1}},\left\{\nu_{j}\right\}_{j \neq j_{1}} ; p-1, q-1\right) .
$$

ii) Si l'opérateur hypergéométrique $L$ possède des paramètres tels que $\mu_{i_{1}}-\nu_{j_{1}}$ est un entier $<0$, et si l'on choisit les indices de telle sorte que :

$$
\Re \nu_{j_{1}}=\inf \left\{\Re \nu_{j}, \mu_{i_{1}}-\nu_{j} \in \mathbb{Z} \mid \mu_{i_{1}}-\nu_{j}<0\right\},
$$

alors l'opérateur $L$ est équivalent à

$$
L^{\prime}=L\left(\left\{\mu_{i}\right\}_{i \neq i_{1}},\left\{\nu_{j}\right\}_{j \neq j_{1}} ; p-1, q-1\right)\left(\theta+\nu_{j_{1}}-1\right),
$$

et donc au dual de :

$$
\left(\theta-\nu_{j_{1}}+1\right)\left\{z \prod_{i \neq i_{1}}\left(\theta+\mu_{i}+1\right)-\prod_{j \neq j_{1}}\left(\theta-\nu_{j}+1\right)\right\} .
$$

\section{Démonstration}

i) Il est clair que si $\mu_{i_{1}}=\nu_{j_{1}}$, alors :

$$
L=\left(\theta+\nu_{j_{1}}-1\right) L\left(\left\{\mu_{i}\right\}_{i \neq i_{1}},\left\{\nu_{j}\right\}_{j \neq j_{1}} ; p-1, q-1\right) \text {. }
$$

De manière générale si $\mu_{i_{1}}-\nu_{j_{1}}$ est un entier $\geq 0$, et si

$$
\begin{gathered}
\Re \nu_{j_{1}}=\sup \left\{\Re \nu_{j} \mid \mu_{i_{1}}-\nu_{j} \in \mathbb{Z}, \mu_{i_{1}}-\nu_{j} \geq 0\right\}, \\
-316-
\end{gathered}
$$


alors : $\left.\left.\left.\left.\left\{\nu_{j} \in\right]\right] \nu_{j_{1}}, \mu_{i_{1}}\right]\right]\right\}$ est vide et d'après le lemme 1.2.2, en remplaçant $\mu_{i_{1}}$ par $\nu_{j_{1}}$, on obtient que l'opérateur $L$ est équivalent à :

$$
\begin{aligned}
& L\left(\left\{\nu_{j_{1}},\left\{\mu_{i}\right\}_{i \neq i_{1}}\right\},\left\{\nu_{j}\right\}_{j} ; p, q\right)= \\
& \quad=\left(\theta+\nu_{j_{1}}-1\right) L\left(\left\{\mu_{i}\right\}_{i \neq i_{1}},\left\{\nu_{j}\right\}_{j \neq j_{1}} ; p-1, q-1\right) .
\end{aligned}
$$

ii) Il est clair que si $\mu_{i_{1}}=\nu_{j_{1}}-1$, alors :

$$
L=L\left(\left\{\mu_{i}\right\}_{i \neq i_{1}},\left\{\nu_{j}\right\}_{j \neq j_{1}} ; p-1, q-1\right)\left(\theta+\nu_{j_{1}}-1\right) .
$$

Plus généralement si $\mu_{i_{1}}-\nu_{j_{1}}$ est un entier $<-1$, et si

$$
\Re \nu_{j_{1}}=\inf \left\{\Re \nu_{j} \mid \mu_{i_{1}}-\nu_{j} \in \mathbb{Z}, \mu_{i_{1}}-\nu_{j}<0\right\},
$$

alors $\left.\left.\left.\left.\left\{\nu_{j} \in\right]\right] \mu_{i_{1}}, \nu_{j_{1}}-1\right]\right]\right\}$ est vide et d'après le lemme 1.2.2, en remplaçant $\mu_{i_{1}}$ par $\nu_{j_{1}}-1$, on obtient que l'opérateur $L$ est équivalent à

$$
\begin{aligned}
& L\left(\left\{\nu_{j_{1}}-1,\left\{\mu_{i}\right\}_{i \neq i_{1}}\right\},\left\{\nu_{j}\right\}_{j} ; p, q\right)= \\
& \quad=L\left(\left\{\mu_{i}\right\}_{i \neq i_{1}},\left\{\nu_{j}\right\}_{j \neq j_{1}} ; p-1, q-1\right)\left(\theta+\nu_{j_{1}}-1\right) .
\end{aligned}
$$

Proposition 1.2.2. - Considérons l'opérateur hypergéométrique :

$$
L=L\left(\left\{\mu_{i}\right\}_{i},\left\{\nu_{j}\right\}_{j} ; p, q\right) .
$$

S'il existe $s$ indices distincts $i_{1}, \ldots, i_{s}$ et $s$ indices distincts $j_{1}, \ldots, j_{s}$ tels que :

$$
\mu_{i_{k}}-\nu_{j_{k}} \in \mathbb{Z}, \quad \mu_{i_{k}}-\nu_{j_{k}} \geq 0
$$

et si les paramètres sont choisis de telle sorte que :

$$
\mu_{i_{1}}-\nu_{j_{1}}=\inf \left\{\mu_{i}-\nu_{j} \mid \mu_{i}-\nu_{j} \in \mathbb{Z}, \mu_{i}-\nu_{j} \geq 0\right\}
$$

et plus généralement pour $t \leq s$,

$$
\begin{gathered}
\mu_{i_{t}}-\nu_{j_{t}}=\inf \left\{\mu_{i}-\nu_{j} \mid i \notin\left\{i_{1}, \ldots, i_{t-1}\right\}\right. \\
\left.j \notin\left\{j_{1}, \ldots, j_{t-1}\right\}, \mu_{i}-\nu_{j} \in \mathbb{Z}, \mu_{i}-\nu_{j} \geq 0\right\}, \\
-317-
\end{gathered}
$$


alors il existe $s$ nombres $\alpha_{k}$ tels que $\alpha_{k}-\nu_{j_{k}} \in \mathbb{Z}$ et tels que l'opérateur $L$ est équivalent $\grave{a}$ :

$$
L^{\prime}=\prod_{i \in\{1, \ldots, s\}}\left(\theta+\alpha_{i}-1\right) L\left(\left\{\mu_{i}\right\}_{i \notin\left\{i_{1}, \ldots, i_{s}\right\}},\left\{\nu_{j}\right\}_{j \notin\left\{j_{1}, \ldots, j_{s}\right\}} ; p-s, q-s\right) .
$$

Démonstration. - Démontrons la proposition par récurrence sur $s$.

Pour $s=1$, on obtient le résultat grâce à la proposition 1.2.1. Supposons que l'on ait prouvé que l'opérateur $L$ est équivalent à :

$$
L_{1}=\prod_{i \in I}\left(\theta+\alpha_{i}-1\right) L_{1}^{\prime},
$$

où card $I=s-1$, où pour tout $i$ de $I, \alpha_{k}-\nu_{j_{k}} \in \mathbb{Z}$ et

$$
L_{1}^{\prime}=L\left(\left\{\mu_{i}\right\}_{i \notin\left\{i_{1}, \ldots, i_{s-1}\right\}},\left\{\nu_{j}\right\}_{j \notin\left\{j_{1}, \ldots, j_{s-1}\right\}} ; p-s+1, q-s+1\right) .
$$

On a :

$$
L_{1}=L\left(\left\{\mu_{i}^{\prime}\right\}_{i},\left\{\nu_{j}^{\prime}\right\}_{j} ; p, q\right)
$$

où, pour $i \notin\left\{i_{1}, \ldots, i_{s-1}\right\}, \mu_{i}^{\prime}=\mu_{i}$, pour $j \notin\left\{j_{1}, \ldots, j_{s-1}\right\}, \nu_{j}^{\prime}=\nu_{j}$, pour $k \in\{1, \ldots, s-1\}$, on a $\mu_{i_{k}}^{\prime}=\nu_{j_{k}}^{\prime}=\alpha_{k}$.

Premier cas. $-\Re \nu_{j_{s}}^{\prime}=\sup \left\{\Re \nu_{j}^{\prime} \mid \mu_{i_{s}}^{\prime}-\nu_{j}^{\prime} \in \mathbb{Z}, \mu_{i_{s}}^{\prime}-\nu_{j}^{\prime} \geq 0\right\}$, c'est-àdire que $\left.\left.\left.\left.\left\{\nu_{j}^{\prime} \mid \nu_{j}^{\prime} \in\right]\right] \nu_{j_{s}}^{\prime}, \mu_{i_{s}}^{\prime}\right]\right]\right\}$ est vide, alors, d'après le lemme 1.2.2, on peut remplacer $\mu_{i_{s}}^{\prime}$ par $\nu_{j_{s}}=\nu_{j_{s}}^{\prime}$ et l'opérateur $L_{1}$ est équivalent à :

$$
L^{\prime}=\left(\theta+\nu_{j_{s}}-1\right) \prod_{i \in I}\left(\theta+\alpha_{i}-1\right) L\left(\left\{\mu_{i}\right\}_{i \notin\left\{i_{1}, \ldots, i_{s}\right\}},\left\{\nu_{j}\right\}_{j \notin\left\{j_{1}, \ldots, j_{s}\right\}}\right) .
$$

La proposition est alors démontrée au rang $s$ dans ce premier cas.

Deuxième cas. $-\Re \nu_{j_{s}}^{\prime} \neq \sup \left\{\Re \nu_{j}^{\prime} \mid \mu_{i_{s}}^{\prime}-\nu_{j}^{\prime} \in \mathbb{Z}, \mu_{i_{s}}^{\prime}-\nu_{j}^{\prime} \geq 0\right\}$, c'està-dire que $\left.\left.\left.\left.\left\{\nu_{j}^{\prime} \mid \nu_{j}^{\prime} \in\right]\right] \nu_{j_{s}}^{\prime}, \mu_{i_{s}}^{\prime}\right]\right]\right\}$ n'est pas vide, alors, d'après le choix des paramètres $\nu_{j_{s}}\left(=\nu_{j_{s}}^{\prime}\right)$ et $\mu_{i_{s}}\left(=\mu_{i_{s}}^{\prime}\right)$, on en déduit pour $j \notin\left\{j_{1}, \ldots, j_{s-1}\right\}$, $\left.\left.\left.\left.\nu_{j}^{\prime}=\nu_{j} \notin\right]\right] \nu_{j_{s}}^{\prime}, \mu_{i_{s}}^{\prime}\right]\right]$. Il existe donc des $\alpha_{k_{n}} \in\left[\left[\nu_{j_{s}}^{\prime}, \mu_{i_{s}}^{\prime}\right]\right]$ et on peut écrire :

$$
\prod_{i \in I}\left(\theta+\alpha_{i}-1\right)=\prod_{n \in\{1, \ldots, h\}}\left(\theta+\alpha_{k_{n}}-1\right)^{p_{n}} \prod_{i \in K}\left(\theta+\alpha_{i}-1\right)
$$

avec $p_{n}>0$ et $\Re \mu_{i_{s}} \geq \Re \alpha_{k_{1}}>\Re \alpha_{k_{2}}>\cdots>\Re \alpha_{k_{n}} \geq \Re \nu_{j_{s}}$ et, pour tout $i \in K, \alpha_{i} \notin\left[\left[\nu_{j_{s}}^{\prime}, \mu_{i_{s}}^{\prime}\right]\right]$. 
Pour tout $n \in\{1, \ldots, s\}$, l'opérateur $L_{1}$ possède donc $p_{n}\left(p_{n}>0\right)$ paramètres $\mu$ égaux à $\alpha_{k_{n}}$ et $p_{n}$ paramètres $\nu$ égaux à $\alpha_{k_{n}}$. D'après le lemme 1.2 .2 , on peut remplacer le paramètre $\mu_{i_{s}}$ par $\alpha_{k_{1}}$, puis successivement, pour tout $n \in\{1, \ldots, h\}$, les $p_{n}$ paramètres $\mu$ égaux à $\alpha_{k_{n}}$ et les $p_{n}$ paramètres $\nu$ égaux à $\alpha_{k_{n}}$ par $\alpha_{k_{1}}-n+1$ et finalement, le paramètre $\nu_{j_{s}}$ par $\alpha_{k_{1}}-h+1$.

Soit $L_{2}$ l'opérateur ainsi obtenu et posons $\alpha=\alpha_{k_{1}}$, on a alors :

$$
L_{2}=\prod_{n \in\{1, \ldots, h\}}(\theta+\alpha-n)^{p_{n}} \prod_{i \in K}\left(\theta+\alpha_{i}-1\right) L_{2}^{\prime}
$$

où

$$
\begin{aligned}
L_{2}^{\prime}=L\left(\left\{\alpha,\left\{\mu_{i}\right\}_{i \notin\left\{i_{1}, \ldots, i_{s-1}\right\}}\right\}\right. & \\
& \left.\left\{\alpha-h+1,\left\{\nu_{j}\right\}_{j \notin\left\{j_{1}, \ldots, j_{s-1}\right\}}\right\} ; p-s+1, q-s+1\right) .
\end{aligned}
$$

Pour tout $i \in K$, on a $\alpha_{i} \notin[[\alpha-h+1, \alpha]]$ pour tout $i \notin\left\{i_{1}, \ldots, i_{s-1}\right\}$, on a $\mu_{i} \notin[[\alpha-h+1, \alpha-1]]$ et pour tout $i \notin\left\{j_{1}, \ldots, j_{s-1}\right\}$, on a $\nu_{i} \notin[[\alpha-h+2, \alpha]]$. Alors, d'après le lemme 1.2.3, on obtient un opérateur équivalant en remplaçant $\left(p_{1}, \ldots, p_{h}\right)$ par une suite décroissante d'entiers $\left(p_{1}^{\prime}, \ldots, p_{h}^{\prime}\right)$ vérifiant $\left\{p_{1}^{\prime}, \ldots, p_{h}^{\prime}\right\}=\left\{p_{1}, \ldots, p_{h}\right\}$.

Soit $L_{3}$ l'opérateur ainsi obtenu, on a :

$$
L_{3}=\prod_{n \in\{1, \ldots, h\}}(\theta+\alpha-n)^{p_{n}^{\prime}} \prod_{i \in K}\left(\theta+\alpha_{i}-1\right) L_{2}^{\prime} .
$$

D'après le lemme 1.2.5, l'opérateur $L_{3}$ est équivalent à :

$$
L^{\prime}=(\theta+\alpha-n)^{p_{1}^{\prime}+1} \prod_{n \in\{2, \ldots, h\}}(\theta+\alpha-n)^{p_{n}^{\prime}} \prod_{i \in K}\left(\theta+\alpha_{i}-1\right) L^{0}
$$

où

$$
L^{0}=L\left(\left\{\mu_{i}\right\}_{i \notin\left\{i_{1}, \ldots, i_{s}\right\}},\left\{\nu_{j}\right\}_{j \notin\left\{j_{1}, \ldots, j_{s}\right\}} ; p-s, q-s\right) .
$$

L'opérateur $L$ est donc équivalent à $L^{\prime}$.

L'hypothèse de récurrence est bien vérifiée dans ce deuxième cas. Donc la proposition est démontrée. 
Proposition 1.2.3.- Considérons l'opérateur hypergéométrique :

$$
L=L\left(\left\{\mu_{i}\right\}_{i},\left\{\nu_{j}\right\}_{j} ; p, q\right) .
$$

S'il existe $s$ indices distincts $i_{1}, \ldots, i_{s}$ et $s$ indices distincts $j_{1}, \ldots, j_{s}$, tels que $\mu_{i_{k}}-\nu_{j_{k}} \in \mathbb{Z}, \mu_{i_{k}}-\nu_{j_{k}}<0$, et si les paramètres sont choisis de telle sorte que :

$$
\mu_{i_{1}}-\nu_{j_{1}}=\sup \left\{\mu_{i}-\nu_{j} \mid \mu_{i}-\nu_{j} \in \mathbb{Z}, \mu_{i}-\nu_{j}<0\right\},
$$

et plus généralement, pour $t \leq s$,

$$
\begin{aligned}
\mu_{i_{t}}-\nu_{j_{t}}=\sup \left\{\mu_{i}-\nu_{j} \mid\right. & i \notin\left\{i_{1}, \ldots, i_{t-1}\right\}, \\
j & \left.\notin\left\{j_{1}, \ldots, j_{t-1}\right\}, \mu_{i}-\nu_{j} \in \mathbb{Z}, \mu_{i}-\nu_{j}<0\right\},
\end{aligned}
$$

alors il existe $s$ nombres $\alpha_{k}$ tels que $\alpha_{k}-\nu_{j_{k}} \in \mathbb{Z}$ et tels que l'opérateur $L$ est équivalent $\grave{a}$ :

$$
L^{\prime}=L\left(\left\{\mu_{i}\right\}_{i \notin\left\{i_{1}, \ldots, i_{s}\right\}},\left\{\nu_{j}\right\}_{i \notin\left\{j_{1}, \ldots, j_{s}\right\}} ; p-s, q-s\right) \prod_{i \in\{1, \ldots, s\}}\left(\theta+\alpha_{i}-1\right) .
$$

Démonstration. - Il suffit d'appliquer la proposition précédente à l'opérateur dual de $L$ en utilisant le lemme 1.1.1 et la remarque qui le précède.

Corollaire 1.2.1. - Pour tout opérateur hypergéométrique :

$$
L=L\left(\left\{\mu_{i}\right\}_{i},\left\{\nu_{j}\right\}_{j} ; p, q\right) \text {. }
$$

on a $m_{L} \geq m_{L}^{\prime}$ et $n_{L} \geq n_{L}^{\prime}$.

Proposition 1.2.4.- Considérons l'opérateur hypergéométrique :

$$
L=L\left(\left\{\mu_{i}\right\}_{i},\left\{\nu_{j}\right\}_{j} ; p, q\right) .
$$

S'il existe $s$ indices distincts $i_{1}, \ldots, i_{s}$ et $s$ indices distincts $j_{1}, \ldots, j_{s}$, tels que $\mu_{i_{k}}-\nu_{j_{k}} \in \mathbb{Z}$ et si les paramètres sont choisis de telle sorte que:

$$
\begin{aligned}
\left|\mu_{i_{1}}-\nu_{j_{1}}\right|=\inf & \left\{\left|\mu_{i}-\nu_{j}\right| \mid \mu_{i}-\nu_{j} \in \mathbb{Z}\right\} \\
& -320-
\end{aligned}
$$


et plus généralement, pour $t \leq s$,

$$
\begin{aligned}
\left|\mu_{i_{t}}-\nu_{j_{t}}\right|=\inf \left\{\left|\mu_{i}-\nu_{j}\right| \mid i \notin\left\{i_{1}, \ldots, i_{t-1}\right\},\right. \\
\left.j \notin\left\{j_{1}, \ldots, j_{t-1}\right\}, \mu_{i}-\nu_{j} \in \mathbb{Z}\right\},
\end{aligned}
$$

alors il existe $s$ nombres $\alpha_{k}$ tels que $\alpha_{k}-\nu_{j_{k}} \in \mathbb{Z}$ et tels que l'opérateur $L$ est équivalent $a$ :

$\prod_{i \in I}\left(\theta+\alpha_{i}-1\right) L\left(\left\{\mu_{i}\right\}_{i \notin\left\{i_{1}, \ldots, i_{s}\right\}},\left\{\nu_{j}\right\}_{j \notin\left\{j_{1}, \ldots, j_{s}\right\}} ; p-s, q-s\right) \prod_{i \in J}\left(\theta+\alpha_{i}-1\right)$

avec $I \cup J=\{1, \ldots, s\}$.

Démonstration. - Démontrons la proposition par récurrence sur $s$. Pour $s=1$, on obtient le résultat grâce à la proposition 1.2.1.

Supposons que l'on ait prouvé que l'opérateur $L$ est équivalent à :

$$
L_{1}=\prod_{i \in I^{\prime}}\left(\theta+\alpha_{i}-1\right) L_{1}^{\prime} \prod_{i \in J^{\prime}}\left(\theta+\alpha_{i}-1\right),
$$

où $\operatorname{card}\left(I^{\prime} \cup J^{\prime}\right)=s-1$, où pour tout $i$ de $I^{\prime} \cup J^{\prime}, \alpha_{i}-\nu_{j_{i}} \in \mathbb{Z}$, et où :

$$
L_{1}^{\prime}=L\left(\left\{\mu_{i}\right\}_{i \notin\left\{i_{1}, \ldots, i_{s-1}\right\}},\left\{\nu_{j}\right\}_{j \notin\left\{j_{1}, \ldots, j_{s-1}\right\}} ; p-s+1, q-s+1\right) .
$$

On a :

$$
L_{1}=L\left(\left\{\mu_{i}^{\prime}\right\}_{i},\left\{\nu_{j}^{\prime}\right\}_{j} ; p, q\right)
$$

où :

- pour $i \notin\left\{i_{1}, \ldots, i_{s-1}\right\}, \mu_{i}^{\prime}=\mu_{i}$,

- pour $j \notin\left\{j_{1}, \ldots, j_{s-1}\right\}, \nu_{j}^{\prime}=\nu_{j}$,

- pour $i \in\left\{i_{1}, \ldots, i_{s-1}\right\}$, il existe $k \in I^{\prime}$ tel que $\mu_{i}^{\prime}=\alpha_{k}$ ou $k \in J^{\prime}$ tel que $\mu_{i}^{\prime}=\alpha_{k}-1$

- pour $j \notin\left\{j_{1}, \ldots, j_{s-1}\right\}$, il existe $h \in I^{\prime} \cup J^{\prime}$ tel que $\nu_{j}^{\prime}=\alpha_{h}$.

On peut supposer $\mu_{i_{s}}^{\prime}-\nu_{j}^{\prime} \geq 0$ car sinon, on se ramène à l'équation duale.

Premier cas. $-\Re \nu_{j_{s}}^{\prime}=\sup \left\{\Re \nu_{j}^{\prime} \mid \mu_{i_{s}}^{\prime}-\nu_{j}^{\prime} \in \mathbb{Z}, \mu_{i_{s}}^{\prime}-\nu_{j}^{\prime} \geq 0\right\}$ c'est-àdire que $\left.\left.\left.\left.\left\{\nu_{j}^{\prime} \mid \nu_{j}^{\prime} \in\right]\right] \nu_{j_{s}}^{\prime}, \mu_{i_{s}}^{\prime}\right]\right]\right\}$ est vide, alors, d'après le lemme 1.2.2, on peut remplacer $\mu_{i_{s}}^{\prime}$ par $\nu_{j_{s}}=\nu_{j_{s}}^{\prime}$ et l'opérateur $L_{1}$ est équivalent à :

$$
\left(\theta+\nu_{j_{s}}-1\right) \prod_{i \in I^{\prime}}\left(\theta+\alpha_{i}-1\right) L\left(\left\{\mu_{i}\right\}_{i \notin\left\{i_{1}, \ldots, i_{s}\right\}},\left\{\nu_{j}\right\}_{i \notin\left\{j_{1}, \ldots, j_{s}\right\}}\right) \prod_{i \in J^{\prime}}\left(\theta+\alpha_{i}-1\right) .
$$

La proposition est alors démontrée au rang $s$ dans ce premier cas. 
Deuxième cas. $-\Re \nu_{j_{s}}^{\prime} \neq \sup \left\{\Re \nu_{j}^{\prime} \mid \mu_{i_{s}}^{\prime}-\nu_{j}^{\prime} \in \mathbb{Z}, \mu_{i_{s}}^{\prime}-\nu_{j}^{\prime} \geq 0\right\}$, c'està-dire que $\left.\left.\left.\left.\left\{\nu_{j}^{\prime} \mid \nu_{j}^{\prime} \in\right]\right] \nu_{j_{s}}^{\prime}, \mu_{i_{s}}^{\prime}\right]\right]\right\}$ n'est pas vide, alors d'après le choix des paramètres $\nu_{j_{s}}\left(=\nu_{j_{s}}^{\prime}\right)$ et $\mu_{i_{s}}\left(=\mu_{i_{s}}^{\prime}\right)$, comme dans la démonstration précédente, on peut écrire :

$$
\begin{aligned}
& \prod_{i \in I^{\prime}}\left(\theta+\alpha_{i}-1\right)=\prod_{n \in\{1, \ldots, h\}}\left(\theta+\alpha_{k_{n}}-1\right)^{p_{n}} \prod_{i \in K}\left(\theta+\alpha_{i}-1\right) \\
& \prod_{i \in J^{\prime}}\left(\theta+\alpha_{i}-1\right)=\prod_{n \in\{1, \ldots, h\}}\left(\theta+\alpha_{k_{n}}-1\right)^{q_{n}} \prod_{i \in K^{\prime}}\left(\theta+\alpha_{i}-1\right)
\end{aligned}
$$

avec $p_{n}+q_{n}>0$ et $\Re \mu_{i_{s}} \geq \Re \alpha_{k_{1}}>\Re \alpha_{k_{2}}>\cdots>\Re \alpha_{k_{h}} \geq \Re \nu_{j_{s}}$ et, pour tout $i \in K \cup K^{\prime}, \alpha_{i} \notin\left[\left[\nu_{j_{s}}^{\prime}, \mu_{i_{s}}^{\prime}\right]\right]$.

Pour tout $n \in\{1, \ldots, s\}$, l'opérateur $L_{1}$ possède donc $p_{n}\left(p_{n} \geq 0\right)$ paramètres $\mu$ égaux à $\alpha_{k_{n}}, q_{n}\left(q_{n} \geq 0\right)$ paramètres $\mu$ égaux à $\alpha_{k_{n}}-1$ et $p_{n}+q_{n}\left(p_{n}+q_{n}>0\right)$ paramètres $\nu$ égaux à $\alpha_{k_{n}}$.

D'après le lemme 1.2 .2 , on peut remplacer le paramètre $\mu_{i_{s}}$ par $\alpha_{k_{1}}$, le paramètre $\nu_{j_{s}}$ par $\alpha_{k_{h}}$ et, successivement, pour tout $n \in\{2, \ldots, h\}$, si $p_{n}>0$, les $p_{n}$ paramètres $\mu$ égaux à $\alpha_{k_{n}}$ puis les $p_{n}+q_{n}$ paramètres $\nu$ égaux à $\alpha_{k_{n}}$ par $\alpha_{k_{1}}-n+1$ et enfin si $q_{n}>0$, les $q_{n}$ paramètres $\mu$ égaux à $\alpha_{k_{n}}-1$ par $\alpha_{k_{1}}-n$. Puis, si $q_{h}>0$, on remplace le paramètre $\nu_{j_{s}}$ par $\alpha_{k_{1}}-h$ et si $q_{h}=0$, on remplace le paramètre $\nu_{j_{s}}$ par $\alpha_{k_{1}}-h+1$.

Soit $L_{2}$ l'opérateur ainsi obtenu et posons dans toute la suite $\alpha=\alpha_{k_{1}}$; $P^{\prime}=\prod_{i \in K}\left(\theta+\alpha_{i}\right)$ et $R^{\prime}=\prod_{i \in K^{\prime}}\left(\theta+\alpha_{i}\right)$. On a :

$$
L_{2}=\prod_{n \in\{1, \ldots, h\}}(\theta+\alpha-n)^{p_{n}} P^{\prime} L_{2}^{\prime} \prod_{n \in\{1, \ldots, h\}}(\theta+\alpha-n)^{q_{n}} R^{\prime}
$$

avec si $q_{h}=0$ :

$$
\begin{aligned}
L_{2}^{\prime}=L\left(\left\{\alpha,\left\{\mu_{i}\right\}_{i \notin\left\{i_{1}, \ldots, i_{s-1}\right\}}\right\},\right. & \\
& \left.\left\{\alpha-h+1,\left\{\nu_{j}\right\}_{j \notin\left\{j_{1}, \ldots, j_{s-1}\right\}}\right\} ; p-s+1, q-s+1\right)
\end{aligned}
$$

et si $q_{h}>0$ :

$$
\begin{aligned}
L_{2}^{\prime}=L & \left(\left\{\alpha,\left\{\mu_{i}\right\}_{i \notin\left\{i_{1}, \ldots, i_{s-1}\right\}}\right\},\right. \\
& \left.\left\{\alpha-h,\left\{\nu_{j}\right\}_{i \notin\left\{j_{1}, \ldots, j_{s-1}\right\}}\right\} ; p-s+1, q-s+1\right) .
\end{aligned}
$$

Ensuite, on peut remplacer, d'après le lemme 1.2.3, la suite $\left(p_{1}, \ldots, p_{h}\right)$ par $\left(p_{1}^{\prime}, \ldots, p_{h}^{\prime}\right)$ vérifiant $\left\{p_{1}^{\prime}, \ldots, p_{h}^{\prime}\right\}=\left\{p_{1}, \ldots, p_{h}\right\}$ et telle que si $q_{i}=0$, on 
a $p_{i}^{\prime} \geq p_{i+1}^{\prime}$ puisque l'opérateur $L_{2}$ possède exactement $p_{i+1}$ paramètres $\mu$ égaux à $\alpha-i$ et $p_{i}$ paramètres $\nu$ égaux à $\alpha-i+1$.

Enfin d'après le lemme 1.2.4, si $q_{i}=0$, on peut remplacer un paramètre $\nu$ égal à $\alpha-i$ par $\alpha-i+1$.

Soit $L_{3}$ l'opérateur ainsi obtenu et soit $J^{\prime \prime}=\left\{i / q_{i} \neq 0\right\}$. On a alors :

$$
\begin{aligned}
L_{3}= & (\theta+\alpha-1)^{p_{1}^{\prime}+1} \prod_{2 \leq i \leq s}(\theta+\alpha-i)^{p_{i}^{\prime}} \times \\
& \times \prod_{i \in J^{\prime \prime}}(\theta+\alpha-i-1) P^{\prime} L_{3}^{\prime} R^{\prime} \prod_{i \in J^{\prime \prime}}(\theta+\alpha-i-1)^{q_{i}-1}
\end{aligned}
$$

où

$$
L_{3}^{\prime}=L\left(\left\{\mu_{i}\right\}_{i \notin\left\{i_{1}, \ldots, i_{s}\right\}},\left\{\nu_{j}\right\}_{j \notin\left\{j_{1}, \ldots, j_{s}\right\}} ; p-s, q-s\right) .
$$

L'hypothèse de récurrence est bien vérifiée dans ce deuxième cas. Donc la proposition est démontrée.

Corollaire 1.2.2. - Pour tout opérateur hypergéométrique :

$$
L=L\left(\left\{\mu_{i}\right\}_{i},\left\{\nu_{j}\right\}_{j} ; p, q\right)
$$

on a $r_{L}=r_{L}^{\prime}$.

Démonstration. - Appliquons la proposition précédente à $s=r_{L}^{\prime}$. Alors

$$
\begin{aligned}
\prod_{i \in I}\left(\theta+\alpha_{i}-1\right) L\left(\left\{\mu_{i}\right\}_{i \notin\left\{i_{1}, \ldots, i_{s}\right\}},\right. & \\
& \left.\left\{\nu_{j}\right\}_{j \notin\left\{j_{1}, \ldots, j_{s}\right\}} ; p-s, q-s\right) \prod_{i \in J}\left(\theta+\alpha_{i}-1\right)
\end{aligned}
$$

avec $I \cup J=\{1, \ldots, s\}$ est un produit de $r_{L}^{\prime}+1$ opérateurs irréductibles puisque d'après la définition de $r_{L}^{\prime}$, pour tout $i \notin\left\{i_{1}, \ldots, i_{r_{L}^{\prime}}\right\}$ et tout $j \notin\left\{j_{1}, \ldots, j_{r_{L}^{\prime}}\right\}$, on a $\mu_{i}-\nu_{j} \notin \mathbb{Z}$ et donc d'après le théorème 1.1.1, l'opérateur

$$
L\left(\left\{\mu_{i}\right\}_{i \notin\left\{i_{1}, \ldots, i_{s}\right\}},\left\{\nu_{j}\right\}_{j \notin\left\{j_{1}, \ldots, j_{s}\right\}} ; p-s, q-s\right)
$$

est irréductible. 
D'après la remarque 1.2.1, la collection des classes d'équivalence des opérateurs irréductibles divisant un opérateur donné est unique et donc leur nombre aussi.

Remarque. - Katz [Ka] a montré le résultat de ce lemme sans donner de construction effective de décomposition de l'opérateur.

Proposition 1.2.5. - Soit

$$
L\left(\left\{\mu_{i}\right\}_{i \in I},\left\{\nu_{j}\right\}_{j \in J} ; p, q\right),
$$

un opérateur hypergéométrique. Si l'opérateur $L$ admet $k$ solutions linéairement indépendantes dans la $\mathbb{K}$-algèbre engendrée par $\log z$ et les $z^{\alpha}$ où les $\alpha$ parcourent $\mathbb{C}$, alors il existe $k$ indices distincts $i_{1}, \ldots, i_{k}$ dans $I$ et $k$ indices distincts $j_{1}, \ldots, j_{k}$ dans $J$ tels que pour tout $s, 1 \leq s \leq k$ :

$$
\mu_{i_{s}}-\nu_{j_{s}} \in \mathbb{Z}, \quad \mu_{i_{s}}-\nu_{j_{s}}<0 .
$$

Démonstration. - Soit $V$ la $\mathbb{K}$-algèbre mentionnée dans la proposition.

Démontrons le résultat par récurrence sur $k$. Supposons $k=1$.

Comme $V$ est invariant sous la monodromie, l'opérateur $L$ est divisible à droite par un opérateur $L_{1}$ à coefficients dans $\mathbb{K}$ dont toutes les solutions sont dans $V$. En effet, le $\mathbb{C}$-espace vectoriel de solutions de $L$ engendré par l'orbite de la solution sous l'action de la monodromie est contenue dans $V$ et est stable sous la monodromie donc cet espace est l'espace de solutions d'un opérateur à coefficients dans $V$ et stable sous la monodromie et donc à coefficients dans $\mathbb{K}$. D'après le lemme 1.2.6, l'opérateur $L_{1}$ possède une solution $f$ de la forme $z^{\alpha}[P(z)+C F(z)]$ où $F$ est une fonction hypergéométrique, $C$ une constante et $P$ un polynôme.

Puisque $f$ appartient à $V$, si $C \neq 0, F$ est aussi un polynôme et ceci n'est possible que s'il existe $i$ et $j$ tels que $\mu_{i}-\nu_{j} \in \mathbb{Z}, \mu_{i}-\nu_{j}<0$.

Si $C=0$, alors l'opérateur

$$
L^{\prime}=L\left(\left\{\mu_{i}-\alpha\right\}_{i \in I},\left\{\nu_{j}-\alpha\right\}_{j \in J} ; p, q\right)
$$

admet une solution polynomiale et on aboutit à la même conclusion en considérant les équations indicielles en zéro et en l'infini de $L^{\prime}$.

La propriété est donc démontrée pour $k=1$, supposons la prouvée à l'ordre $k-1$. Si $L$ admet $k$ solutions dans $V$, elle en admet $k-1$. 
D'après l'hypothèse de récurrence, il existe $k-1$ indices $i_{1}, \ldots, i_{k-1}$ dans $I$ et $k-1$ indices $j_{1}, \ldots, j_{k-1}$ dans $J$, tels que pour tout $s, 1 \leq s \leq k-1$ : $\mu_{i_{s}}-\nu_{j_{s}} \in \mathbb{Z}, \mu_{i_{s}}-\nu_{j_{s}}<0$.

D'après la proposition 1.2.3, si les indices sont choisis de telle sorte que :

$$
\begin{aligned}
\mu_{i_{s}}-\nu_{j_{s}}=\sup \left\{\mu_{i}-\nu_{j} \mid\right. & i \notin\left\{i_{1}, \ldots, i_{s-1}\right\}, \\
j & \left.\notin\left\{j_{1}, \ldots, j_{s-1}\right\}, \mu_{i}-\nu_{j} \in \mathbb{Z}, \mu_{i}-\nu_{j}<0\right\},
\end{aligned}
$$

il existe $k-1$ nombres complexes $\gamma_{i}$ tels que l'opérateur $L$ est isomorphe à :

$$
L_{1}=L\left(\left\{\mu_{i}\right\}_{i \in I^{*}},\left\{\nu_{j}\right\}_{j \in J^{*}} ; p-k+1, q-k+1\right) \prod_{i \in\{1, \ldots, k-1\}}\left(\theta+\gamma_{i}-1\right)
$$

où $I^{*}=I \backslash\left\{i_{1}, \ldots, i_{k-1}\right\}, J^{*}=J \backslash\left\{j_{1}, \ldots, j_{k-1}\right\}$. Les opérateurs $L$ et $L_{1}$ étant équivalents, l'opérateur $L_{1}$ admet aussi $k$ solutions linéairement indépendantes dans $V$ dont une n'est pas solution de $\prod_{i \in\{1, \ldots, k-1\}}\left(\theta+\gamma_{i}\right)$.

Soit $f$ cette solution, et soit $g=\prod_{i \in\{1, \ldots, k-1\}}\left(\theta+\gamma_{i}\right)(f)$, alors $g$ appartient à $V$ et il est solution de l'opérateur

$$
L\left(\left\{\mu_{i}\right\}_{i \in I^{*}},\left\{\nu_{j}\right\}_{j \in J^{*}} ; p-k+1, q-k+1\right) .
$$

On est ramené au cas où $k=1$. Il existe donc $i_{k} \notin\left\{i, \ldots, i_{k-1}\right\}, j_{k} \notin$ $\left\{j, \ldots, j_{k-1}\right\}$ tels que $\mu_{i_{k}}-\nu_{j_{k}}<0$. La proposition est donc démontrée.

CoRollaire 1.2.3.- Pour tout opérateur hypergéométrique :

$$
L=L\left(\left\{\mu_{i}\right\}_{i},\left\{\nu_{j}\right\}_{j} ; p, q\right)
$$

on a $n_{L}^{\prime} \geq n_{L}^{\prime \prime}$

Corollaire 1.2.4. - Pour tout opérateur hypergéométrique :

$$
L=L\left(\left\{\mu_{i}\right\}_{i},\left\{\nu_{j}\right\}_{j} ; p, q\right)
$$

on a $n_{L}=n_{L}^{\prime}=n_{L}^{\prime \prime}$ et $m_{L}=m_{L}^{\prime}$.

Démonstration. - D'après les corollaires 1.2 .1 et 1.2 .3 , on a $n_{L} \geq n_{L}^{\prime} \geq$ $n_{L}^{\prime \prime}$. Mais $n_{L}^{\prime \prime} \geq n_{L}$, car si $L$ est isomorphe à un opérateur $L^{\prime}$ divisible à droite par $\left(\theta+\alpha_{1}\right) \cdots\left(\theta+\alpha_{n_{L}}\right)$ où les $\alpha_{i}$ sont des nombres complexes, l'opérateur $L^{\prime}$ admet $n_{L}$ solutions indépendantes dans l'espace $V$ de dimension infinie 
engendré par $\left\{z^{\alpha_{i}}\right\}$ et $\log z$ et par conséquent l'opérateur $L$ aussi. On en déduit que $n_{L}=n_{L}^{\prime}=n_{L}^{\prime \prime}$. En appliquant ce résultat à l'opérateur dual de $L$, on obtient $m_{L}=m_{L}^{\prime}$.

Remarque. - La difficulté d'obtenir une décomposition d'un opérateur $L$ (voir [Ka1]) provient du fait que, si l'on a déja décomposé l'opérateur $L$ en produit de plusieurs opérateur $\Pi L_{i}$ dont l'un $L_{j}$ est réductible, on ne peut pas, pour poursuivre la décomposition de $L$ à équivalence près, décomposer à équivalence près l'opérateur $L_{j}$ sans considérer tout le produit $\prod L_{i}$. Par exemple, considérons l'opérateur $\left(\theta^{2}-\theta\right) \theta$. L'opérateur $\theta^{2}-\theta=(\theta-1) \theta$ est isomorphe à $(\theta-1)(\theta-2)$ mais $\left(\theta^{2}-\theta\right) \theta$ n'est pas isomorphe à $(\theta-1)(\theta-2) \theta$. Considérons de même les opérateurs de l'exemple du paragraphe 1.2.2. On a :

$$
L=(\theta-1) L(\{\alpha\},\{\beta, \gamma, \delta\} ; 1,3) \theta
$$

et

$$
L^{\prime}=(\theta-1) L(\{-1, \alpha\},\{1, \beta, \gamma, \delta\} ; 2,4)
$$

avec d'après le lemme 1.2.2, $L(\{-1, \alpha\},\{1, \beta, \gamma, \delta\} ; 2,4)$ équivalent à $L(\{\alpha\},\{\beta, \gamma, \delta\} ; 1,3) \theta$ alors que les opérateurs :

$$
L^{\prime}=L(\{\alpha\},\{\beta, \gamma, \delta\} ; 1,3) \theta(\theta-1)
$$

et

$$
L=(\theta-1) L(\{\alpha\},\{\beta, \gamma, \delta\} ; 1,3) \theta
$$

ne sont pas équivalents car $n_{L} \neq n_{L^{\prime}}$.

1.2.4 Propriété des opérateurs vérifiant les conditions $C(m+n, m, n)$

Proposition 1.2.7. - Si l'opérateur hypergéométrique

$$
L=L\left(\left\{\mu_{i}\right\}_{i},\left\{\nu_{j}\right\}_{j} ; p, q\right)
$$

vérifie les conditions $C(m+n, m, n)$ et s'il est équivalent au produit d'opérateurs irréductibles :

$$
L^{\prime}=\prod_{i \in I}\left(\theta+\alpha_{i}-1\right) L\left(\left\{\mu_{i}\right\}_{i \in I^{*}},\left\{\nu_{j}\right\}_{j \in J^{*}} ; p^{\prime}, q^{\prime}\right) \prod_{j \in J}\left(\theta+\alpha_{j}-1\right)
$$

alors : 
a) pour tout couple $(i, j)$ de $I \times I$ ou de $J \times J$, soit $\alpha_{i}=\alpha_{j}$, soit $\alpha_{i}-\alpha_{j} \notin \mathbb{Z}$

b) pour tout couple $(i, j)$ de $I \times I^{*}$ ou $\mu_{j}-\alpha_{i} \in \mathbb{Z}$ et $\mu_{j}-\alpha_{i} \geq 0$ ou $\mu_{j}-\alpha_{i} \notin \mathbb{Z}$

c) pour tout couple $(i, j)$ de $I \times J^{*}$ ou $\alpha_{i}-\nu_{j} \in \mathbb{Z}$ et $\alpha_{i}-\nu_{j} \geq 0$ ou $\alpha_{i}-\nu_{j} \notin \mathbb{Z}$

d) pour tout couple $(i, j)$ de $I^{*} \times J$ ou $\mu_{i}-\alpha_{j} \in \mathbb{Z}$ et $\mu_{i}-\alpha_{j}<0$ ou $\mu_{i}-\alpha_{j} \notin \mathbb{Z}$

e) pour tout couple $(i, j)$ de $J^{*} \times J$ ou $\alpha_{j}-\nu_{i} \in \mathbb{Z}$ et $\alpha_{j}-\nu_{i} \leq 0$ ou $\alpha_{j}-\nu_{i} \notin \mathbb{Z}$.

Démonstration. - On peut supposer, si la conclusion n'est pas réalisée, en se ramenant à l'autre cas par dualité, qu'il existe un couple $(i, j)$ de $I \times I$ tels que $\alpha_{i} \neq \alpha_{j}$ et $\alpha_{i}-\alpha_{j} \in \mathbb{Z}$. On aurait alors $\Re \alpha_{i}<\Re \alpha_{j}$ ou $\Re \alpha_{j}<\Re \alpha_{i}$. Du théorème 1.2.1, on déduit que le nombre d'opérateurs de la forme $(\theta+\alpha)$ qui divisent $L^{\prime}$ est strictement plus grand que $n$. Ce qui contredit évidemment que l'opérateur $L^{\prime}$ vérifie les conditions $C(m+n, m, n)$. L'assertion a) est donc prouvée.

On démontre de la même manière les autres assertions.

THÉORÈME 1.2.2. - Soient

$$
L=L\left(\left\{\mu_{i}\right\}_{i},\left\{\nu_{j}\right\}_{j} ; p, q\right) \quad \text { et } \quad L^{\prime}=L\left(\left\{\mu_{i}^{\prime}\right\}_{i},\left\{\nu_{j}^{\prime}\right\}_{j} ; p, q\right)
$$

des opérateurs hypergéométriques vérifiant les conditions $C(m+n, m, n)$ et respectivement isomorphes aux opérateurs

$$
\prod_{i \in I}\left(\theta+\alpha_{i}-1\right) \mathbf{L} \prod_{j \in J}\left(\theta+\alpha_{j}-1\right)=P \mathbf{L} R
$$

et

$$
\prod_{i \in I^{\prime}}\left(\theta+\alpha_{i}^{\prime}-1\right) \mathbf{L}^{\prime} \prod_{j \in J^{\prime}}\left(\theta+\alpha_{j}^{\prime}-1\right)=P^{\prime} \mathbf{L}^{\prime} R^{\prime}
$$

où $\mathbf{L}$ et $\mathbf{L}^{\prime}$ sont des opérateurs hypergéométriques irréductibles d'ordre $q-m-n$.

Alors les opérateurs $L$ et $L^{\prime}$ sont isomorphes si et seulement si, d'une part, les opérateurs $P, \mathbf{L}, R$ sont respectivement isomorphes aux opérateurs $P^{\prime}, \mathbf{L}^{\prime}, R^{\prime}$ et, d'autre part, pour tous paramètres $\alpha_{i}, \alpha_{j}, \alpha_{k}^{\prime}, \alpha_{h}^{\prime}$, ò̀ 
$(i, j, h, k)$ appartient $\grave{a} I \times J \times I^{\prime} \times J^{\prime}$, de la même classe de congruence modulo $\mathbb{Z}$, les différences $\alpha_{i}-\alpha_{j}$ et $\alpha_{h}^{\prime}-\alpha_{k}^{\prime}$ sont de même signe, en convenant que 0 est de signe positif.

\section{Démonstration}

- Montrons que les conditions sont suffisantes. Posons $L_{1}=P \mathbf{L} R$ et $L_{1}^{\prime}=P^{\prime} \mathbf{L}^{\prime} R^{\prime}$ où

$$
\mathbf{L}=L\left(\left\{\mu_{i}\right\}_{i \in I^{*}},\left\{\nu_{j}\right\}_{j \in J^{*}} ; p-r, q-r\right)
$$

et

$$
\mathbf{L}^{\prime}=L\left(\left\{\mu_{i}^{\prime}\right\}_{i \in I^{\prime *}},\left\{\nu_{j}^{\prime}\right\}_{j \in J^{\prime *}} ; p-r, q-r\right)
$$

Soit $\left\{\gamma_{i}\right\}_{i \in S}$, un système de représentants des classes modulo $\mathbb{Z}$ des paramètres de $L_{1}$. Soit :

$$
\left\{\alpha_{i_{1}}, \ldots, \alpha_{i_{f}}, \mu_{h_{1}}, \ldots, \mu_{h_{s}}, \nu_{k_{1}}, \ldots, \mu_{k_{t}}, \alpha_{j_{1}}, \ldots, \alpha_{j_{g}}\right\}
$$

l'ensemble des paramètres de $L_{1}$ de la classe de $\gamma$ où $\gamma \in\left\{\gamma_{i}\right\}_{i \in S}$. On a $s t=0$ car $\mathbf{L}$ est irréductible.

On dira :

a) $\gamma$ est de type $e_{1}(f, s, t, g)$ si un seul élément de $\{f, s, t, g\}$ est non nul;

b) $\gamma$ est de type $2(f, s, g)$ si $t=0$, deux éléments au moins de $\{f, s, g\}$ sont non nuls et si, lorsque $f g \neq 0$ alors la relation suivante est satisfaite :

$$
\Re \alpha_{i_{1}}=\cdots=\Re \alpha_{i_{f}}<\Re \alpha_{j_{1}}=\cdots=\Re \alpha_{j_{g}}
$$

c) $\gamma$ est de type $3(f, t, g)$ si $s=0$, deux éléments au moins de $\{f, t, g\}$ sont non nuls et si, lorsque $f g \neq 0$ alors la relation suivante est satisfaite :

$$
\Re \alpha_{i_{1}}=\cdots=\Re \alpha_{i_{f}} \geq \Re \alpha_{j_{1}}=\cdots=\Re \alpha_{j_{g}} .
$$

D'après la proposition précédente, l'opérateur $L_{1}$ vérifiant les conditions $C(m+n, m, n)$, s'il existe des paramètres $\alpha_{i}, \alpha_{j}, \alpha_{k}^{\prime}, \alpha_{h}^{\prime}$ où $(i, j, h, k)$ appartient à $I \times J \times I^{\prime} \times J^{\prime}$, dans la même classe de congruence modulo $\mathbb{Z}$ et congrus à au moins un paramètre de $\mathbf{L}$, alors les différences $\alpha_{i}-\alpha_{j}$ et $\alpha_{h}^{\prime}-\alpha_{k}^{\prime}$ sont nécessairement de même signe. On en déduit que chaque élément $\gamma \in\left\{\gamma_{i}\right\}_{i \in S}$ est d'un type et d'un seul. 
Les hypothèses du théorème entraînent que les opérateurs $L_{1}$ et $L_{1}^{\prime}$ ont même système de représentants des classes modulo $\mathbb{Z}$ des paramètres : $\left\{\gamma_{i}\right\}_{i \in S}$ et chaque élément $\gamma \in\left\{\gamma_{i}\right\}_{i \in S}$ est du même type pour $L_{1}$ et $L_{1}^{\prime}$.

Montrons en utilisant le lemme 1.2.2 qu'on obtient un opérateur équivalent à $L_{1}$ en remplaçant tous les paramètres de $L_{1}$ congrus à $\gamma$ modulo $\mathbb{Z}$ par les paramètres de $L_{1}^{\prime}$ correspondants.

Supposons que $\gamma$ est de type $2(f, s, g)$. On a :

$$
\Re \alpha_{i_{1}}=\cdots=\Re \alpha_{i_{f}} \leq \Re \mu_{h_{1}} \leq \cdots \leq \Re \mu_{h_{s}}<\Re \alpha_{j_{1}}=\cdots=\Re \alpha_{j_{g}}
$$

et, on a de même :

$$
\Re \alpha_{i_{1}^{\prime}}^{\prime}=\cdots=\Re \alpha_{i_{f}^{\prime}}^{\prime} \leq \Re \mu_{h_{1}^{\prime}}^{\prime} \leq \cdots \leq \Re \mu_{h_{s}^{\prime}}^{\prime}<\Re \alpha_{j_{1}^{\prime}}^{\prime}=\cdots=\Re \alpha_{j_{g}^{\prime}}^{\prime} .
$$

On obtient d'abord un opérateur équivalent à $L_{1}$, grâce au lemme 1.2.2, en remplaçant sucessivement les $s$ paramètres $\mu_{h_{i}}$ respectivement par $\mu_{h_{i}}^{\prime \prime}=$ $\alpha_{j_{1}}-1$ puis les $f$ paramètres $\mu$ égaux à $\alpha_{i_{1}}$ par $\alpha_{j_{1}}-1$ et enfin les $f$ paramètres $\nu$ égaux à $\alpha_{i_{1}}$ par $\alpha_{j_{1}}-1$. Ainsi les $f$ paramètres $\alpha_{i}$, pour $i \in I$ sont remplacés par $\alpha_{i}^{\prime \prime}=\alpha_{j_{1}}-1$. Soit $L_{2}$, l'opérateur ainsi obtenu.

Dans ces conditions, on a soit $\Re \alpha_{j_{1}} \leq \Re \alpha_{j_{1}^{\prime}}^{\prime}$, soit $\Re \alpha_{j_{1}}>\Re \alpha_{j_{1}^{\prime}}^{\prime}$ et alors $\Re \alpha_{i_{1}^{\prime}}^{\prime}<\Re \alpha_{i_{1}}^{\prime \prime}$.

Si $\Re \alpha_{j_{1}} \leq \Re \alpha_{j_{1}^{\prime}}^{\prime}$, on obtient, d'après le lemme 1.2.2, un opérateur équivalent à $L_{2}$, et donc à $L_{1}$, en remplaçant successivement les $g$ paramètres $\nu$ égaux à $\alpha_{j_{1}}$ par $\alpha_{j_{1}^{\prime}}^{\prime}$, les $g$ paramètres $\mu$ égaux à $\alpha_{j_{1}}-1$ par $\alpha_{j_{1}^{\prime}}^{\prime}-1$, les $s$ paramètres $\mu_{h_{1}}^{\prime \prime}, \ldots, \mu_{h_{s}}^{\prime \prime}$ respectivement par $\mu_{h_{1}^{\prime}}^{\prime}, \ldots, \mu_{h_{s}^{\prime}}^{\prime}$, les $f$ paramètres $\mu$ égaux à $\alpha_{i_{1}}^{\prime \prime}$ par $\alpha_{i_{1}^{\prime}}^{\prime}$, et finalement les $f$ paramètres $\nu$ égaux à $\alpha_{i_{1}}^{\prime \prime} \operatorname{par} \alpha_{i_{1}^{\prime}}^{\prime}$.

Si $\Re \alpha_{j_{1}}>\Re \alpha_{j_{1}^{\prime}}^{\prime}$, on obtient, d'après le lemme 1.2.2, un opérateur équivalent à $L_{2}$, et donc à $L_{1}$, en remplaçant sucessivement les $f$ paramètres $\nu$ égaux à $\alpha_{i_{1}}^{\prime \prime}$ par $\alpha_{i_{1}^{\prime}}^{\prime}$, les $f$ paramètres $\mu$ égaux à $\alpha_{i_{1}}^{\prime \prime}$ par $\alpha_{i_{1}^{\prime}}^{\prime}$, les $s$ paramètres $\mu_{h_{1}}^{\prime \prime}, \ldots, \mu_{h_{s}}^{\prime \prime}$ respectivement par $\mu_{h_{1}^{\prime}}^{\prime}, \ldots, \mu_{h_{s}^{\prime}}^{\prime}$, les $g$ paramètres $\mu$ égaux à $\alpha_{j_{1}}-1$ par $\alpha_{j_{1}^{\prime}}^{\prime}-1$, et finalement les $g$ paramètres $\nu$ égaux à $\alpha_{j_{1}}$ par $\alpha_{j_{1}^{\prime}}^{\prime}$

On a montré ainsi que, si $\gamma$ est de type 2, on obtient un opérateur équivalent à $L_{1}$ en remplaçant tous les paramètres de $L_{1}$ congrus à $\gamma$ modulo $\mathbb{Z}$ par les paramètres de $L_{1}^{\prime}$ correspondants. Les mêmes arguments permettent de conclure dans le cas où $\gamma$ est de type 3 ou de type 1 . 
En réitérant l'opération pour toutes les classes de congruence, on montre ainsi que les opérateurs $L_{1}$ et $L_{1}^{\prime}$ sont équivalents. On a donc prouvé que les conditions étaient suffisantes.

- Montrons qu'elles sont nécessaires. Il est évident que les opérateurs $P, \mathbf{L}$, $R$ doivent être respectivement isomorphes aux opérateurs $P^{\prime}, \mathbf{L}^{\prime}, R^{\prime}$. Posons, comme précédemment, $L_{1}=P \mathbf{L} R$ et $L_{1}^{\prime}=P^{\prime} \mathbf{L}^{\prime} R^{\prime}$. On montre, en utilisant le lemme 1.2.2 et les mêmes arguments que ceux de la démonstration cidessus, que l'on peut se ramener au cas où $\mathbf{L}=\mathbf{L}^{\prime}, R=R^{\prime}$. Supposons qu'il existe des paramètres $\alpha_{i}, \alpha_{j}, \alpha_{h}^{\prime}, \alpha_{k}^{\prime}$ dans la même classe de congruence modulo $\mathbb{Z}$, où $(i, j, h, k)$ appartient à $I \times J \times I^{\prime} \times J^{\prime}$, tels que les différences $\alpha_{i}-\alpha_{j}$ et $\alpha_{h}^{\prime}-\alpha_{k}^{\prime}$ ne sont pas de même signe. La seule possibilité est que $\alpha_{i}$ est du type ${ }_{2}(s, 0, t)$ pour l'un des opérateurs et du type $e_{3}(s, 0, t)$ pour l'autre.

Supposons que $\alpha_{i}-\alpha_{j} \geq 0$, tandis que $\alpha_{h}^{\prime}-\alpha_{k}^{\prime}<0$.

On peut remplacer, d'après le lemme 1.2.2, dans l'opérateur $L_{1}$, les paramètres $\nu$ égaux à $\alpha_{i}$ par $\alpha_{j}$, puis, les paramètres $\mu$ égaux à $\alpha_{i}$ par $\alpha_{j}$.

De même, puisque $\alpha_{k}^{\prime}=\alpha_{j}$, on peut remplacer dans l'opérateur $L_{1}^{\prime}$ les paramètres $\mu$ égaux à $\alpha_{h}^{\prime}$ par $\alpha_{j}-1$, puis, les paramètres $\nu$ égaux à $\alpha_{h}^{\prime}$ par $\alpha_{j}-1$.

On est donc ramené au cas où :

$$
L_{1}=P^{\prime \prime}\left(\theta+\alpha_{j}-1\right)^{s} \mathbf{L}\left(\theta+\alpha_{j}-1\right)^{t} R^{\prime \prime}
$$

et

$$
L_{1}^{\prime}=P^{\prime \prime \prime}\left(\theta+\alpha_{j}-2\right)^{s} \mathbf{L}\left(\theta+\alpha_{j}-1\right)^{t} R^{\prime \prime}
$$

avec aucun paramètre de $P^{\prime \prime}, R^{\prime \prime}$ et $\mathbf{L}$ congru à $\alpha_{j}$ modulo $\mathbb{Z}$.

Nous allons montrer qu'il existe au moins une solution de l'opérateur $L_{1}$ qui ne peut pas s'écrire comme combinaison linéaire à coefficients dans $K$ des solutions de $L_{1}^{\prime}$ et de leurs dérivées. Cela prouvera que les opérateurs $L_{1}$ et $L_{1}^{\prime}$ ne sont pas équivalents.

Comme $-\alpha_{j}+1$ est la seule racine congrue à $-\alpha_{j}$ modulo $\mathbb{Z}$ de l'équation indicielle en zéro de $L_{1}$, l'opérateur $L_{1}$ admet $s+t$ solutions $F_{i}, 1 \leq i \leq s+t$ de la forme $Y_{1}, Y_{1} \log z+Y_{2}, Y_{1}(\log z)^{2}+2 Y_{2} \log z+Y_{3}$, etc., où $Y_{i}$ est le produit de $\varphi$ par une fonction holomorphe en zéro où l'on a posé [Po] :

$$
\begin{gathered}
\varphi(z)=z^{1-\alpha_{j}} . \\
-330-
\end{gathered}
$$




\section{Opérateurs hypergéométriques réductibles}

Comme $\left(\varphi, \ldots, \varphi(\log z)^{t-1}\right)$ est une base de solutions de $\left(\theta+\alpha_{j}-1\right)^{t}$. Ces $t$ solutions sont donc $t$ solutions linéairement indépendantes de $L_{1}$ et on peut choisir

$$
\left(F_{1}, \ldots, F_{t}\right)=\left(\varphi, \ldots, \varphi(\log z)^{t-1}\right)
$$

et

$$
F_{t+1}=\varphi(\log z)^{t}+\varphi A_{1,1}
$$

où $A_{1,1}$ est une fonction holomorphe en zéro puis jusqu'à :

$$
F_{t+s}=\varphi(\log z)^{t+s-1}+\varphi \sum_{0 \leq g \leq s-1}(\log z)^{g} A_{s, g}
$$

où les $A_{i, j}$ sont des fonctions holømorphes en zéro.

Comme $-\alpha_{j}+2$ est la racine congrue à $-\alpha_{j}$ modulo $\mathbb{Z}$ de l'équation indicielle en zéro de $L_{1}^{\prime}$ admettant la plus grande partie réelle, l'opérateur $L_{1}^{\prime}$ admet $s$ solutions linéairement indépendantes $G_{m}, 1 \leq m \leq s$, de la forme [Po] : $Z_{1}, Z_{1} \log z+Z_{2}, Z_{1}(\log z)^{2}+2 Z_{2} \log z+Z_{3}$, etc., où $Z_{i}$ est le produit de $z \varphi$ par une fonction holomorphe en zéro.

D'autre part, $\left(F_{1}, \ldots, F_{t}\right)=\left(\varphi, \ldots, \varphi(\log z)^{t-1}\right)$ est une base de solutions de $\left(\theta+\alpha_{j}-1\right)^{t}$ et forme donc $t$ solutions linéairement indépendantes de $L_{1}^{\prime}$. Les $F_{i}, 1 \leq i \leq t$ et les $G_{m}$ sont clairement linéairement indépendantes sur $\mathbb{C}$. De plus, comme l'équation indicielle en zéro de l'opérateur $L_{1}^{\prime}$ admet exactement $t+s$ solutions congrues à $-\alpha_{j}$ modulo $\mathbb{Z}$, toute solution de $L_{1}^{\prime}$ d'exposant congru à $-\alpha_{j}$ est une combinaison linéaire à coefficients dans $\mathbb{C}$ des $F_{i}, 1 \leq i \leq t$ et $\operatorname{des} G_{m}$.

Si $F_{t+s}$ était une combinaison linéaire à coefficients dans $\mathbb{K}$ des solutions de $L_{1}^{\prime}$ et de leurs dérivées, comme son exposant est $-\alpha_{j}$, elle serait une combinaison linéaire à coefficients dans $\mathbb{K}$ des $F_{i}, 1 \leq i \leq t$, des $G_{m}$ et de leurs dérivées. On aurait alors :

$$
\varphi(\log z)^{t+s-1}=\varphi \sum_{0 \leq g \leq s-1} z(\log z)^{g} C_{g}+\varphi \sum_{0 \leq g \leq t-1}(\log z)^{g} D_{g}
$$

où les $C_{g}$ et les $D_{g}$ sont des produits d'un éléments de $\mathbb{K}$ par une fonction holomorphe en zéro. Or, cette relation est impossible (il suffit de considérer la clôture de Zariski du groupe de monodromie en zéro), donc le théorème est démontré. 


\section{Calculs de quelques groupes de Galois d'opérateurs hypergéométriques vérifiant les conditions $C(m+n, m, n)$}

\subsection{Rappels et notations}

\subsubsection{Généralités}

DÉFInITION 2.1.1. - Si $F$ est un corps différentiel et $K$ un sous-corps de $F$, on appelle groupe de Galois différentiel de $F / K$, l'ensemble des automorphismes de $(F / K)$ qui commutent avec la dérivation $\delta$ de $F$. On note:

$$
\operatorname{Gal}_{\text {diff }}(F / K)=\{\sigma \in \operatorname{Aut}(F / K) \mid \forall x \in F, \delta(\sigma(x))=\sigma(\delta x)\} .
$$

DÉFInITION 2.1.2.-Étant donné un corps différentiel $(K, \delta)$ et une extension différentielle $(F / K)$, on dit que $F$ est une extension de PicardVessiot de $K$, s'il existe un opérateur différentiel $L \in K[\delta]$ :

$$
L=a_{n} \delta^{n}+a_{n-1} \delta^{n-1}+\cdots+\alpha_{1} \delta+a_{0}
$$

avec $a_{i} \in K, i=0, \ldots, n$, et un ensemble de solutions $y_{1}, \ldots, y_{n}$, dans $F$, de l'équation $L y=0$, linéairement indépendantes sur le corps $C_{F}$ des constantes de $F$ et vérifiant:

i) $C_{K}=C_{F}$,

ii) $F=K\left(\delta^{k}\left(y_{i}\right), i=1, \ldots, n\right.$ et $\left.k \in \mathbb{N}\right)$.

Proposition 2.1.1 (Kolchin [K, chap. 3]). - Si K est de caractéristique nulle, et si $C_{K}$ est algébriquement clos, il existe, pour tout $L \in K[\delta]$, une extension de Picard-Vessiot associée à $L$, unique à $K$-isomorphisme près.

Pour toute extension différentielle intermédiaire $N \supset M \supset K$, on pose $M^{\prime}=\operatorname{Gal}_{\text {diff }}(N / M)$ et pour tout sous-groupe $H$ de $\mathrm{Gal}_{\text {diff }}(N / K)$, on note $H^{\prime}$ le sous-corps différentiel de $N$ formé des éléments fixés par $H$ :

$$
H^{\prime}=\{x \in N \mid \forall u \in H, u(x)=x\} .
$$


On dira que $H$ (resp. $M$ ) est fermé (au sens galoisien) si $\left(H^{\prime}\right)^{\prime}=H$ (resp. $\left.(M)^{\prime}=M\right)$. On dira que l'extension $N / K$ est normale si $K$ est fermé dans $N / K$.

THÉORÈME 2.1.1 [K, théorème 5.9]. - Soit $K$ un corps différentiel de caractéristique 0 avec un corps des constantes algébriquement clos et $N$ une extension de Picard-Vessiot de K.

i) Le groupe $G=\mathrm{Gal}_{\mathrm{diff}}(N / K)$ est un groupe algébrique et la correspondance de Galois usuelle établit une bijection entre les sous-groupes algébriques de $G$ et les extensions différentielles intermédiaires de $\mathrm{N} / \mathrm{K}$. En particulier les sous-groupes fermés de $G$ au sens galoisien en sont les sous-groupes algébriques.

ii) Un sous-groupe fermé $H$ de $G$ est normal si et seulement si le corps $H^{\prime}$ est normal sur $K$ et dans ce cas $G / H=\operatorname{Gal}_{\text {diff }}\left(H^{\prime} / K\right)$ et $H^{\prime} / K$ est une extension de Picard-Vessiot.

Pour tout opérateur différentiel $M$ à coefficients dans le corps $\mathbb{K}$, on désigne par $F_{M}$ l'extension de Picard-Vessiot de $M$, par $V_{M}$ son espace de solutions dans $F_{M}$, par $G_{M}=\operatorname{Gal}_{\text {diff }}\left(F_{M} / \mathbb{K}\right)$ son groupe de Galois différentiel. C'est à un sous-groupe algébrique de $\operatorname{Aut}\left(V_{M}\right)$ que le groupe $G_{M}$ s'identifie naturellement.

La composante neutre du groupe algébrique $G_{M}$, c'est-à-dire la composante connexe qui contient l'identité sera notée $\left(G_{M}\right)^{0}$.

On notera $\left(G_{M}\right)^{0, \text { der }}$ le groupe dérivé de $\left(G_{M}\right)^{0}$, c'est-à-dire le plus petit sous-groupe normal de $\left(G_{M}\right)^{0}$ tel que $\left(G_{M}\right)^{0} /\left(G_{M}\right)^{0 \text {,der }}$ est commutatif. Si $M_{1}, \ldots, M_{n}$ désignent $n$ opérateurs différentiels à coefficients dans le corps $K$, on notera

$$
\prod_{i=1, \ldots, n} F_{M_{i}}=F_{M_{1}} \cdots F_{M_{n}}
$$

le compositum des extensions $F_{M_{i}}, 1 \leq i \leq n$, c'est-à-dire le plus petit corps contenant les extensions $F_{M_{i}}, 1 \leq i \leq n\left(\prod_{i=1, \ldots, n} F_{M_{i}}\right.$ est une extension de Picard-Vessiot).

D'après la proposition 1.1.1, il est clair que l'on a le résultat suivant.

Proposition 2.1.2. - Les groupes de Galois de deux opérateurs équivalents sont isomorphes. 
DÉFINITION 2.1.3. - Un opérateur différentiel $M$ est dit Lie-irréductible si la composante neutre de son groupe de Galois $\left(G_{M}\right)^{0}$ opère irréductiblement sur $V_{M}$.

Les deux lemmes qui suivent reprennent des énoncés classiques de la théorie de Galois. Les démonstrations utilisent des arguments analogues.

Lemme 2.1.1.- Soient $L$ un corps différentiel, $N / L$ une extension de Picard-Vessiot et $M / L$ une extension différentielle contenue dans une extension de Picard-Vessiot de L. Alors :

i) $M N / M$ est une extension de Picard-Vessiot;

ii) si l'extension $M$ est linéairement disjointe de $N$ sur le corps $L$ alors $M \cap N=L$;

iii) l'extension $M$ est linérairement disjointe de $N$ sur le corps $M \cap N$.

Remarque. - Si, de plus $M / L$ est aussi une extension de Picard-Vessiot, les propositions : " $M$ est linéairement disjointe de $N$ sur $L$ " et " $N$ est linéairement disjointe de $M$ sur $L$ " sont donc équivalentes. On dira alors que $M$ et $N$ sont linéairement disjointes sur $L$.

Lemme 2.1.2. - Soient $L$ un corps différentiel, $N / L$ une extension de Picard-Vessiot et $M / L$ une extension différentielle contenue dans une extension de Picard-Vessiot de L. Alors le groupe $\operatorname{Gal}_{\text {diff }}(M N / M)$ est isomorphe à $\mathrm{Gal}_{\text {diff }}(N / M \cap N)$ et, en particulier, les propositions suivantes sont équivalentes :

i) $M \cap N=L$;

ii) le groupe $\mathrm{Gal}_{\operatorname{diff}}(M N / M)$ est isomorphe à $\operatorname{Gal}_{\operatorname{diff}}(N / L)$.

\subsubsection{Opérateurs hypergéométriques irréductibles}

Rappelons que si $q, p$, deux entiers tels que $q>p \geq 0$ et $\left\{\mu_{1}, \ldots, \mu_{p}\right\}$, $\left\{\nu_{1}, \ldots, \nu_{q}\right\}$ deux familles de nombres complexes, on appelle opérateur hypergéométrique, confluent généralisé associé à ces données l'opérateur différentiel d'ordre $q$ :

$$
L=L\left(\left\{\mu_{i}\right\}_{i},\left\{\nu_{j}\right\}_{j} ; p, q\right)=(-1)^{q-p} z \prod_{i=1, \ldots, p}\left(\theta+\mu_{i}\right)-\prod_{i=1, \ldots, q}\left(\theta+\nu_{j}-1\right) .
$$

où $\theta=z(\mathrm{~d} / \mathrm{d} z)$. 
Si $\beta$ est un nombre complexe, notons $L \otimes z^{\beta}$, l'opérateur

$$
L \otimes z^{\beta}=L\left(\left\{\mu_{i}+\beta\right\}_{i},\left\{\nu_{j}+\beta\right\}_{j} ; p, q\right) .
$$

Nous reproduisons maintenant les résultats de $[\mathrm{BBH}]$ et [Ka] qui sont, soit nécessaires pour la suite, soit qu'ils permettent, joints à nos résultats, de lire le groupe de Galois sur les paramètres de l'opérateur.

Proposition 2.1.3 ([Ka, théorème 3.4]). - L'opérateur

$$
L\left(\left\{\mu_{i}\right\}_{i},\left\{\nu_{j}\right\}_{j} ; p, q\right)
$$

irréductible est isomorphe à son dual si et seulement si les conditions suivantes sont réalisées:

i) $q-p$ est pair;

ii) il existe une permutation $i \mapsto i^{\prime}$ de $[1, \ldots, p]$ telle que $\mu_{i}+\mu_{i^{\prime}} \in \mathbb{Z}$;

iii) il existe une permutation $j \mapsto j^{\prime}$ de $[1, \ldots, q]$ telle que $\nu_{j}+\nu_{j^{\prime}} \in \mathbb{Z}$.

DÉfinition 2.1.4. - L'opérateur $L\left(\left\{\mu_{i}\right\}_{i},\left\{\nu_{j}\right\}_{j} ; p, q\right)$ est dit “Kummer induit" si et seulement si, il existe un entier $d \geq 2$ divisant $a ̀$ la fois $p$ et $q$ et des éléments $A_{1}, \ldots, A_{p / d}$ et $B_{1}, \ldots, B_{q / d}$ de $\mathbb{C}$ tels que :

$$
\begin{aligned}
& \left\{\mu_{i}\right\}=\left\{\frac{A_{i}-j}{d}\right\}_{\substack{i=1, \ldots, p / d \\
j=0, \ldots, d-1}} \bmod \mathbb{Z}, \\
& \left\{\nu_{j}\right\}=\left\{\frac{B_{i}+j}{d}\right\}_{\substack{i=1, \ldots, q / d \\
j=0, \ldots, d-1}} \bmod \mathbb{Z} .
\end{aligned}
$$

Proposition 2.1.4 ([Ka, lemmes 3.5.6 et 3.5.7]). - L'opérateur hypergéométrique confluent $L$ est Lie-irréductible si et seulement si ses paramètres ne sont pas "Kummer induits".

THÉORÈME 2.1.2 ([Ka, théorème 3.6]). - Supposons l'opérateur

$$
L=L\left(\left\{\mu_{i}\right\}_{i},\left\{\nu_{j}\right\}_{j} ; p, q\right)
$$

Lie-irréductible et confluent, on obtient les résultats suivants : 
i) si $q-p$ est impair, alors $\left(G_{L}\right)^{0, \mathrm{der}}$ est $\mathrm{SL}_{q}(\mathbb{C})$. Si $q-p=1$, alors $G_{L}$ est $\mathrm{GL}_{q}(\mathbb{C})$;

ii) si $q-p$ est pair, alors $\left(G_{L}\right)^{0, \mathrm{der}}$ est $\mathrm{SL}_{q}(\mathbb{C})$ ou $\mathrm{SO}_{q}(\mathbb{C})$ ou $\mathrm{Sp}_{q}(\mathbb{C})$ ou, encore lorsque $q-p=6, q=7,8$ ou 9 , l'un des groupes "exceptionnels".

THÉORÈME 2.1.3 (lemme A.2; [Ka, corol. 3.6.1]). - Supposons l'opérateur

$$
L=L\left(\left\{\mu_{i}\right\}_{i},\left\{\nu_{j}\right\}_{j} ; p, q\right)
$$

Lie-irréductible et confluent.

i) Si l'opérateur $L=L\left(\left\{\mu_{i}\right\}_{i},\left\{\nu_{j}\right\}_{j} ; p, q\right)$ est isomorphe à son dual, alors, le groupe $G_{L}$ est un sous-groupe d'un groupe orthogonal ou symplectique et c'est un sous-groupe d'un groupe symplectique si et seulement si : q est pair et $\gamma=\sum \nu_{j}-\sum \mu_{i} \in \mathbb{Z}$.

ii) Le groupe $\left(G_{L}\right)^{0, \mathrm{der}}$ est contenu dans $\mathrm{SO}_{q}(\mathbb{C})$ ou $\mathrm{Sp}_{q}(\mathbb{C})$ si et seulement s'il existe $\beta$ tel que l'opérateur

$$
L \otimes z^{\beta}=L\left(\left\{\mu_{i}+\beta\right\}_{i},\left\{\nu_{j}+\beta\right\}_{j} ; p, q\right)
$$

est isomorphe à son dual. De plus, si q est impair, il existe $\beta$ tel que le groupe de Galois de l'opérateur $L \otimes z^{\beta}$ est contenu dans $\mathrm{SO}(q)$.

Pour les détails concernant les cas exceptionnels, voir [Ka] et ainsi que [Mi].

2.2 Le groupe de Galois sous les conditions $C(m+n, m, n)$ avec $m n=0$

\subsubsection{Notations}

Ces notations sont également valables pour le paragraphe 2.3.

Soit $Q$ un opérateur différentiel et $Q_{1} \cdots Q_{s}$ une décomposition de $Q$ en produit d'opérateurs irréductibles.

L'opérateur $Q_{2} \cdots Q_{s}$ applique $V_{Q}$ sur $V_{Q_{1}}$, ainsi, le corps différentiel $F_{Q_{1}}$ est inclus dans $F_{Q}$. Pour tout $i, 1 \leq i \leq s-1$, l'espace vectoriel $V_{Q_{i+1} \cdots Q_{1}}$ étant inclus dans $V_{Q}$, on vérifie que chacun des corps $F_{Q_{i}}$ est 
inclus dans $F_{Q}$. D'après la remarque 1.2.1., la collection des corps $F_{Q_{i}}$ ne dépend pas de la décomposition en produit d'opérateurs irréductibles choisie. On peut donc noter sans ambiguïté :

$$
G^{s s}(Q)=\operatorname{Gal}_{\text {diff }}\left(\prod_{1 \leq i \leq s} F_{Q_{i}} / K\right) .
$$

On note par ailleurs

$$
G^{u}(Q)=\operatorname{Gal}_{\text {diff }}\left(F_{Q} / \prod_{1 \leq i \leq s} F_{Q_{i}}\right) .
$$

Le groupe $G_{Q}$ est ainsi une extension de $G^{s s}(Q)$ par le groupe $G^{u}(Q)$.

Considérons un opérateur hypergéométrique vérifiant les conditions $C(m+n, m, n)$ :

$$
L=\prod_{i \in I}\left(\theta+\alpha_{i}-1\right) \mathbf{L} \prod_{j \in J}\left(\theta+\alpha_{j}-1\right)=\prod_{i \in I} P_{i} \mathbf{L} \prod_{j \in J} P_{j}=P \mathbf{L} R
$$

où, pour tout $i \in I \cup J, \alpha_{i}$ est un nombre complexe et $\mathbf{L}$ un opérateur hypergéométrique irréductible.

On convient que si $m=0$ ou $n=0$, on pose respectivement $P=1$, $R=1$. Pour un tel opérateur le groupe $G^{u}(L)$ défini ci-dessus est lui-même une extension du groupe :

$$
K^{u}(L)=\mathrm{Gal}_{\text {diff }}\left(F_{\mathbf{L}} F_{P} F_{R} / \prod_{i \in I \cup J} F_{P_{i}} \times F_{\mathbf{L}}\right)
$$

par le groupe

$$
H^{u}(L)=\operatorname{Gal}_{\text {diff }}\left(F_{L} / F_{P} F_{R} F_{\mathbf{L}}\right) .
$$

(Rappelons, théorème 1.2.1, que les classes d'équivalences de $P$ et de $R$ ne dépendent que de $L$.)

La description du groupe $G^{s s}(L)$ est délicate [Bou1] et nous ferons quelques remarques sur ce groupe au paragraphe 2.2.5.

La partie importante est la description du groupe $G^{u}(L)$. Le groupe $K^{u}(L)$ est isomorphe à $\mathbb{C}$ ou est nul suivant que $\log z$ appartient ou non à $F_{P} F_{R}$ et $\log z$ appartient à $F_{P} F_{R}$ si et seulement si $L$ est divisible à isomorphisme près par un carré de la forme $(\theta+\alpha)^{2}$. 


\subsection{2 Énoncés des résultats}

THÉORÈME 2.2.1. - Soit $L$ un opérateur hypergéométrique vérifiant les conditions $C(r, r, 0)$ ou $C(r, 0, r)$ et tel que un opérateur hypergéométrique irréductible qui le divise à isomorphisme près soit Lie-irréductible. Alors, le groupe $H^{u}(L)$ est isomorphe à $\mathbb{C}^{r(q-r)}$.

Soit, de plus, $\prod_{i \in I}\left(\theta+\alpha_{i}-1\right)$ un opérateur d'ordre $r$ divisant $L \grave{a}$ isomorphisme près. Si les $\alpha_{i}$ sont distincts deux à deux, alors le groupe $G^{u}(L)$ est isomorphe à $\mathbb{C}^{r(q-r)}$ et, sinon, il est isomorphe à $\mathbb{C}^{r(q-r)+1}$

La démonstration de ce théorème fait l'objet des paragraphes 2.2.3 et 2.2.4.

\subsubsection{Le groupe $H^{u}(L)$}

\section{Proposition 2.2.1}

a) Sous les conditions $C(r, r, 0)$, le groupe $H^{u}(L)$ est isomorphe $\grave{a}$ $\operatorname{Hom}\left(V_{P}, V_{\mathbf{L}}\right)$ et donc à $\mathbb{C}^{r(q-r)}$. De plus, si $T=\prod_{i \in I^{*}}\left(\theta+\beta_{i}\right)$, alors le groupe $\mathrm{Gal}_{\mathrm{diff}}\left(F_{L} F_{T} / F_{P} F_{\mathbf{L}} F_{T}\right)$ est encore isomorphe $\grave{a} H^{u}(L)$ et donc à $\mathbb{C}^{r(q-r)}$.

b) Sous les conditions $C(r, 0, r)$, le groupe $H^{u}(L)$ est isomorphe à $\operatorname{Hom}\left(V_{\mathbf{L}}, V_{R}\right)$ et donc à $\mathbb{C}^{r(q-r)}$. De plus, si $T=\prod_{i \in I^{*}}\left(\theta+\beta_{i}\right)$, alors le groupe $\mathrm{Gal}_{\mathrm{diff}}\left(F_{L} F_{T} / F_{P} F_{\mathbf{L}} F_{T}\right)$ est encore isomorphe à $H^{u}(L)$ et donc à $\mathbb{C}^{r(q-r)}$.

Avant de démontrer cette proposition démontrons deux lemmes qui nous serons utiles pour la suite.

LEMme 2.2.1. - Soit $P$ un produit d'opérateurs de la forme $P_{i}=$ $\theta+\alpha_{i}-1$ et

$$
\mathbf{L}=L\left(\left\{\mu_{i}\right\}_{i \in I^{\prime}},\left\{\nu_{j}\right\}_{j \in J^{\prime}} ; p^{\prime}, q^{\prime}\right)
$$

un opérateur hypergéométrique Lie-irréductible.

Alors le groupe $\mathrm{Gal}_{\operatorname{diff}}\left(F_{\mathbf{L}} F_{P} / F_{p}\right)$ contient $\left(G_{\mathbf{L}}\right)^{0, \mathrm{der}}$ et l'espace des solutions $V_{\mathbf{L}}$ est $\mathrm{Gal}_{\operatorname{diff}}\left(F_{\mathbf{L}} F_{P} / F_{p}\right)$-irréductible.

Démonstration. - Appelons $J$ le groupe $\operatorname{Gal}_{\text {diff }}\left(F_{\mathbf{L}} F_{P} / F_{p}\right)$. D'après le lemme 2.1.2, il est isomorphe au groupe $\operatorname{Gal}_{\text {diff }}\left(F_{\mathbf{L}} / F_{\mathbf{L}} \cap F_{p}\right)$. 
Si les opérateurs $P_{i}$ sont distincts deux à deux, alors les extensions $F_{P}$ et $\prod_{i \in I} F_{P_{i}}$ sont égales, sinon on a l'égalité :

$$
F_{P}=\prod_{i \in I} F_{P_{i}}(\log z)
$$

L'opérateur $\mathbf{L}$ étant Lie-irréductible, le groupe $\left(G_{\mathbf{L}}\right)^{0 \text {,der }}$ est réductif, il ne contient, par conséquent, aucun quotient réductif et donc aucun quotient isomorphe à $G_{a}$.

On a ainsi

$$
F_{\mathbf{L}} \cap \prod_{i \in I} F_{P_{i}}(\log z)=F_{\mathbf{L}} \cap \prod_{i \in I} F_{P_{i}}
$$

On peut donc supposer que les opérateurs $P_{i}$ sont distincts deux à deux. Le groupe $\operatorname{Gal}_{\text {diff }}\left(F_{\mathbf{L}} \cap F_{P} / \mathbb{K}\right)$ est un groupe multiplicatif. Considérons alors l'application $\chi$ de $G_{\mathbf{L}}$ dans $\operatorname{Gal}_{\text {diff }}\left(F_{\mathbf{L}} \cap F_{P} / \mathbb{K}\right)$ qui, à tout élément $\sigma$ de $G_{\mathbf{L}}$, associe sa restriction à $F_{\mathbf{L}} \cap F_{P}$. L'application $\chi$ est un caractère et son noyau est le groupe $J$. Un groupe de commutateurs de $G_{\mathbf{L}}$ étant inclus dans le noyau de tout caractère, on en déduit qu'en particulier, le groupe $\left(G_{\mathbf{L}}\right)^{0, \text { der }}$ est contenu dans $J$.

L'opérateur $\mathbf{L}$ étant Lie-irréductible, l'espace $V_{\mathbf{L}}$ est $\left(G_{\mathbf{L}}\right)^{0, \text { der }}$-irréductible et par conséquent $J$-irréductible.

Lemme 2.2.2. - Soient $P$ et $Q$ des opérateurs différentiels et $F$ une extension de Picard-Vessiot. Soit $\varphi=\left(\varphi_{1}, \ldots, \varphi_{s}\right)$ une base de l'espace $V_{P}$ et soit $f=\left(f_{1}, \ldots, f_{s}\right)$ où, pour tout $i \in\{1, \ldots, s\}, f_{i}$ est un élément de $V_{P Q}$ vérifiant $Q\left(f_{i}\right)=\varphi_{i}$.

Notons $\xi_{f}=\left(\xi_{f_{1}}, \ldots, \xi_{f_{s}}\right)$ l'application de $\mathrm{Gal}_{\text {diff }}\left(F_{P Q} F / F_{P} F_{Q} F\right)$ dans $\left(V_{Q}\right)^{s}$ définie par $\xi_{f}(\sigma)=\sigma(f)-f$. Posons

$$
M=\operatorname{Gal}_{\operatorname{diff}}\left(F_{P Q} F / F_{P} F_{Q} F\right) \quad \text { et } \quad N=\operatorname{Gal}_{\operatorname{diff}}\left(F_{P} F_{Q} F / F_{P} F\right) .
$$

a) L'application $\xi_{f}$ définit un homomorphisme injectif de $M$ dans $\left(V_{Q}\right)^{s}$ ou dans $\operatorname{Hom}\left(V_{P}, V_{P Q}\right)$ qui permet d'identifier $M$ à un sous-espace de $\left(V_{Q}\right)^{s}$. De plus, $M$ peut être considéré comme un sous- $N$-module de $\left(V_{Q}\right)^{s}$.

b) Supposons que $N$ opère irréductiblement sur $V_{Q}$. Alors $M$ est distinct de $\left(V_{Q}\right)^{s}$ si et seulement s'il existe un élément non nul $\varphi$ de $V_{P}$ et un élément $\mathbf{f}$ de $F_{P} F$ vérifiant $Q \mathbf{f}=\varphi$. 


\section{Démonstration}

a) L'application $\xi_{f_{i}}$ qui, à tout $\sigma$ de $M$, associe $\sigma\left(f_{i}\right)-f_{i}$, définit un homomorphisme de $M$ dans $V_{Q}$. Elle peut être prolongée de manière naturelle au groupe $H=\operatorname{Gal}_{\text {diff }}\left(F_{P Q} F / F_{P} F\right)$.

Montrons que $\xi_{f} M$ peut être considéré comme un sous- $N$-module de $\left(V_{Q}\right)^{s}$. Soit $f^{\prime}$ une solution de l'équation avec second membre $Q\left(f^{\prime}\right)=\varphi$.

Si $\sigma^{\prime} \in H$, alors $\sigma^{\prime}\left(f^{\prime}\right)=f^{\prime}+\xi_{f}\left(\sigma^{\prime}\right)$, d'où : si $\sigma$ appartient aussi à $H$, on a

$$
\sigma \sigma^{\prime}\left(f^{\prime}\right)=\sigma\left(f^{\prime}\right)+\sigma \xi_{f}\left(\sigma^{\prime}\right)
$$

or $\sigma\left(f^{\prime}\right)=f^{\prime}+\xi_{f}(\sigma)$, donc $\sigma \sigma^{\prime}\left(f^{\prime}\right)=f^{\prime}+\xi_{f}(\sigma)+\sigma \xi_{f}\left(\sigma^{\prime}\right)$, soit enfin :

$$
\xi_{f}\left(\sigma \sigma^{\prime}\right)=\xi_{f}(\sigma)+\sigma \xi_{f}\left(\sigma^{\prime}\right) .
$$

Ainsi, $\xi_{f}$ est un cocycle.

Si $\sigma \in H$ et $\sigma^{\prime} \in M$, alors :

$$
\xi_{f}\left(\sigma \sigma^{\prime} \sigma^{-1}\right)=\xi_{f}(\sigma)+\sigma \xi_{f}\left(\sigma^{\prime} \sigma^{-1}\right) .
$$

Or, si $\sigma^{\prime} \in M$, on a $\xi_{f}\left(\sigma^{\prime} \sigma^{-1}\right)=\xi_{f}\left(\sigma^{\prime}\right)+\xi_{f}\left(\sigma^{-1}\right)$, d'où :

$$
\xi_{f}\left(\sigma \sigma^{\prime} \sigma^{-1}\right)=\xi_{f}(\sigma)+\sigma\left(\xi_{f}\left(\sigma^{\prime}\right)+\xi_{f}\left(\sigma^{-1}\right)\right) .
$$

D'autre part, comme $\sigma \sigma^{-1}=\mathrm{Id}$,

$$
0=\xi_{f}\left(\sigma \sigma^{-1}\right)=\xi_{f}(\sigma)+\sigma \xi_{f}\left(\sigma^{-1}\right)
$$

On obtient finalement que $\xi_{f}\left(\sigma \sigma^{\prime} \sigma^{-1}\right)=\sigma \xi_{f}\left(\sigma^{\prime}\right)$.

Le groupe $M$ étant abélien, $\xi_{f}\left(\sigma \sigma^{\prime} \sigma^{-1}\right)$ ne dépend que de la classe modulo $M$ de $\sigma$. Or

$$
(H / M)=\operatorname{Gal}_{\text {diff }}\left(F_{Q} F_{P} F / F_{P} F\right)=N .
$$

Donc, le groupe $M$, isomorphe à $\xi_{f}(M)$ peut donc ainsi être considéré comme un sous- $N$-module de $\left(V_{Q}\right)^{s}$.

Montrons que l'application $\xi_{f}$ est injective. Il suffit de montrer que son noyau $\operatorname{ker}\left(\xi_{f}\right)$ est réduit à l'application identique. 
Si $\sigma \in \operatorname{ker}\left(\xi_{f}\right)$, alors $\sigma(f)=f$ puisque $\xi_{f}(\sigma)=0$. De plus, comme $\sigma \in M, \sigma$ fixe le corps $F_{Q}$ et donc une base de $V_{Q}$. Or, puisque $\varphi$ est non nul, une base de $V_{Q}$ et $f=\left(f_{1}, \ldots, f_{s}\right)$ constituent une base de $V_{P Q}$. L'élément $\sigma$ fixe $V_{P Q}$ et par suite $F_{P Q}$, l'application $\sigma$ est donc l'identité. Le groupe $M$, isomorphe à $\xi_{f}(M)$, est ainsi un sous- $N$-module de $\left(V_{Q}\right)^{s}$.

b) Comme le groupe $N$ opère irréductiblement sur $V_{Q}$, le groupe $M$ ne peut différer de $\left(V_{Q}\right)^{s}$ que s'il existe des $N$-endomorphismes qui sont, d'après le lemme de Schur, des scalaires $a_{1}, \ldots, a_{s}$ non tous nuls tels que $\xi_{f^{\prime}}(M)$ est nul, où $f^{\prime}$ est un élément de $V_{P Q}$, vérifiant $Q\left(f^{\prime}\right)=\psi$ avec $\psi=\sum_{i} a_{i} \varphi_{i}$.

Supposons que $M$ n'est pas isomorphe à $\operatorname{Hom}\left(V_{P}, V_{Q}\right)$ et soit $f^{\prime}$ l'élément défini ci-dessus. Désignons par $M^{\prime}$ et $H^{\prime}$ respectivement les groupes :

$$
\operatorname{Gal}_{\text {diff }}\left(F_{P} F_{Q} F\left(f^{\prime}\right) / F_{P} F_{Q} F\right) \text { et } \operatorname{Gal}_{\operatorname{diff}}\left(F_{P} F_{Q} F\left(f^{\prime}\right) / F_{P} F\right) \text {. }
$$

Par une démonstration analogue à celle de a), on montre que l'application $\xi_{f^{\prime}}$, définit un homomorphisme injectif de $M^{\prime}$ dans $V_{Q}$ et qu'elle peut être prolongée à $H^{\prime}$. Le groupe $M^{\prime}$, isomorphe à $\xi_{f^{\prime}}\left(M^{\prime}\right)$, peut être alors considéré comme un sous- $N$-module.

On a la suite exacte :

$$
M^{\prime} \longrightarrow H^{\prime} \longrightarrow N
$$

D'après le théorème d'inflation-restriction, on obtient la suite exacte de cohomologie :

$$
H^{1}\left(N, V_{Q}\right) \longrightarrow H^{1}\left(H^{\prime}, V_{Q}\right) \longrightarrow H^{1}\left(M^{\prime}, V_{Q}\right)
$$

Puisque $\xi_{f}\left(M^{\prime}\right)$ est nul, le groupe $M^{\prime}$ est nul. Le groupe de cohomologie $H^{1}\left(M^{\prime}, V_{Q}\right)$ est donc nul aussi.

D'autre part, le groupe $N$ opérant irréductiblement sur $V_{Q}$ par hypothèse, le groupe de cohomologie $H^{1}\left(N, V_{Q}\right)$ est donc nul aussi, [Be].

D'après la suite exacte de cohomomogie, le groupe $H^{1}\left(H, V_{Q}\right)$ est nul. L'application $\xi_{f^{\prime}}$ prolongée à $H^{\prime}$ étant un 1-cocycle, il existe une solution f de l'équation avec second membre $Q(\mathbf{f})=\psi$ telle que le 1-cocycle $\xi_{\mathbf{f}}$ est nul. Cette solution de l'opérateur $P Q$ est donc dans le corps $F_{P} F$; b) est donc démontré, la réciproque étant évidente.

Démonstration de la proposition 2.2.1. - Il suffit de démontrer a). On obtient alors b) en appliquant le résultat précédent à l'opérateur dual. Montrons d'abord que le groupe $H^{u}(L)$ est isomorphe à $\operatorname{Hom}\left(V_{P} ;, V_{\mathbf{L}}\right)$. 
On suppose que $L=P \mathbf{L}$, avec les notations précédentes. Comme le groupe $\mathrm{Gal}_{\text {diff }}\left(F_{\mathbf{L}} F_{P} / F_{P}\right)$ est une extension du groupe $\left(G_{\mathbf{L}}\right)^{\mathbf{0} \text {,der }}$, d'après le lemme 2.2.1, il opère irréductiblement sur $V_{\mathbf{L}}$, alors du lemme 2.2 .2 appliqué à $F=\mathbb{K}$ et à $Q=\mathbf{L}$, on déduit que, si le groupe $H^{u}(L)$ diffère de $\left(V_{\mathbf{L}}\right)^{r}$, il existe un élément non nul $\varphi$ de $V_{P}$, une solution $\mathbf{f}$ dans le corps $F_{P}$ de l'équation avec second membre $\mathbf{L}(\mathbf{f})=\varphi$ et donc il existe une solution de l'opérateur $L$ dans le corps $F_{P}$.

Or, d'après le théorème 1.2.1, ceci est impossible puisque l'opérateur $L$ vérifie les conditions $C(r, r, 0)$. Le groupe $H^{u}(L)$ est donc isomorphe à $\left(V_{\mathbf{L}}\right)^{r}$, et par suite aux groupes $\operatorname{Hom}\left(V_{P}, V_{\mathbf{L}}\right)$ et $\mathbb{C}^{r(q-r)}$.

On déduit, alors, la deuxième partie en utilisant les résultats ci-dessus et les lemmes 2.2 .1 et 2.2.2.

\subsubsection{Le groupe $K^{u}(L)$}

Proposition 2.2.2. - Si l'application $i \mapsto \alpha_{i}$ est injective sur $I$ alors le groupe $K^{u}(L)$ est nul. Sinon, le groupe $K^{u}(L)$ est isomorphe au groupe additif $\mathbb{C}$ noté $G_{a}$. Plus précisément si $P=\prod_{i \in I^{*}}\left(P_{i}\right)^{n_{i}}$ où l'application $i \mapsto \alpha_{i}$ est injective sur $I^{*}$, alors on obtient un isomorphisme de $G_{a}$ sur une représentation matricielle du groupe $K^{u}(L)$ en considérant l'application qui, à tout élément $x$ de $G_{a}$, associe la matrice constituée de blocs diagonaux :

$$
M_{x}=\operatorname{diag}\left(A\left(n_{1}, x\right), \ldots, A\left(n_{s}, x\right)\right)
$$

où

$$
\left\{n_{1}, \ldots, n_{s}\right\}=\bigcup_{i \in I^{*}}\left\{n_{i}\right\}
$$

et où $A\left(n_{i}, x\right)=1$ si $n_{i}=1$, et $A\left(n_{i}, x\right)$ est la matrice carrée triangulaire inférieure d'ordre $n_{i}$ et dont le terme général vérifie :

$$
a_{i j}=\mathbb{C}_{n-j}^{n-i} x^{i-1} \quad \text { si } i>j \text { et } a_{i i}=1 .
$$

Démonstration. - Il suffit d'appliquer les lemmes 2.2.3 et 2.2.4 qui suivent.

Remarque. - Si $P=\prod_{i \in I^{*}}\left(P_{i}\right)^{n_{i}}$ et si $I^{* *}=\{1, \ldots, s\}$, alors une base de solutions de l'opérateur $P$ est

$$
\begin{gathered}
\left\{z^{-\alpha_{1}+1}, z^{-\alpha_{1}+1} \log z, \ldots, z^{-\alpha_{1}+1}(\log z)^{n_{1}-1}, \ldots,\right. \\
\left.z^{-\alpha_{s}+1}, z^{-\alpha_{s}+1} \log z, \ldots, z^{-\alpha_{s}+1}(\log z)^{n_{s}-1}\right\} . \\
-342-
\end{gathered}
$$




\section{Opérateurs hypergéométriques réductibles}

Le groupe $G_{P}$ est isomorphe au groupe des matrices constituées de blocs diagonaux :

$$
\operatorname{diag}\left(A\left(n_{1}, x, \lambda_{1}\right), \ldots, A\left(n_{s}, x, \lambda_{s}\right)\right)
$$

où $\left(\lambda_{1}, \ldots, \lambda_{s}\right)$ est élément de $G\left(\alpha_{1}, \ldots, \alpha_{s}\right)$ et où $A\left(n_{i}, x, \alpha_{i}\right)=\alpha_{i}$ si $n_{i}=1$, et $A\left(n_{i}, x, \alpha_{i}\right)$ est la matrice carrée triangulaire inférieure d'ordre $n_{i}$ et dont le terme général vérifie :

$$
a_{i j}=\mathbb{C}_{n-j}^{n-i} x^{i-1} \quad \text { si } i>j \text { et } a_{i i}=\alpha_{i}
$$

Lemme 2.2.3. - Si $Q$ désigne l'opérateur $\theta+\alpha-1$ et $n$ un entier $>0$, alors le groupe $\mathrm{Gal}_{\operatorname{diff}}\left(F_{Q^{n}} / F_{Q}\right)$ est nul si $n$ est égal à 1 et il est isomorphe au groupe additif $\mathbb{C}$. Dans ce dernier cas, l'application qui, à tout élément $x$ de $\mathbb{C}$, associe la matrice triangulaire inférieure $A(n, x)$ dont le terme général vérifie $a_{i j}=\mathbb{C}_{n-j}^{n-i} x^{i-1}$ si $i>j$ et $a_{i i}=1$, réalise un isomorphisme de $G_{a}$ sur une représentation matricielle du groupe $\operatorname{Gal}_{\text {diff }}\left(F_{Q^{n}} / F_{Q}\right)$.

Démonstration. - Le lemme est évident si $n=1$. Pour $n>1$, le groupe $\operatorname{Gal}_{\text {diff }}\left(F_{Q^{n}} / F_{Q}\right)$ est isomorphe au groupe $G_{\theta^{n}}$ et donc au groupe $\mathrm{Gal}_{\text {diff }}(\mathbb{K}(\log z) / \mathbb{K})$. Une base de solutions de l'opérateur différentiel $\theta^{n}$ est

$$
\left(1, \log z, \ldots,(\log z)^{n-1}\right) .
$$

L'application qui, à tout élément $x$ de $G_{a}$, associe l'élément $\sigma$ du groupe $\mathrm{Gal}_{\text {diff }}(\mathbb{K}(\log z) / \mathbb{K})$ défini par $\sigma(\log z)=1+x \log z$, est un isomorphisme. On déduit le reste du lemme de la relation :

$$
\sigma\left((\log z)^{m}\right)=(\sigma(\log z))^{m} \quad \text { pour } m \in\{1, \ldots, n-1\}
$$

Remarque.- On peut étendre la relation à $n=1$ en écrivant $A(1, x)=1$.

LEMME 2.2.4.- Le groupe $K^{u}(L)$ est isomorphe au groupe :

$$
\operatorname{Gal}_{\text {diff }}\left(F_{P} F_{R} / \prod_{i \in I \cup J} F_{P_{i}}\right) .
$$


Démonstration. - Les extensions $F_{\mathbf{L}}$ et $F_{P} F_{R}$ sont linéairement disjointes sur le corps $\prod_{i \in I \cup J} F_{P_{i}}$ car le groupe $\operatorname{Gal}_{\text {diff }}\left(F_{P} F_{R} / \prod_{i \in I \cup J} F_{P_{i}}\right)$ est soit nul, soit isomorphe au groupe additif $G_{a}$ d'après le lemme 2.2.3; le groupe

$$
\operatorname{Gal}_{\operatorname{diff}}\left(F_{\mathbf{L}} \prod_{i \in I \cup J} F_{P_{i}} / F_{\mathbf{L}} \prod_{i \in I \cup J} F_{P_{i}}\right),
$$

d'après le lemme 2.2.1, opère irréductiblement sur $V_{\mathbf{L}}$, donc est réductif, et ne contient par conséquent aucun quotient réductif et par suite aucun quotient isomorphe à $G_{a}$.

\subsubsection{Remarques sur le groupe $G^{\text {ss }}(L)$}

Notation. - Pour $s$ nombres complexes $\beta_{1}, \ldots, \beta_{s}$, notons $\mathbf{G}\left(\beta_{1}, \ldots\right.$, $\left.\beta_{s}\right)$ l'adhérence de Zariski du groupe engendré dans $\mathbb{C}^{* s}$ par $\exp \left(2 i \pi \beta_{1}\right), \ldots$, $\exp \left(2 i \pi \beta_{s}\right)$. Soit

$$
L=\prod_{i \in I}\left(\theta+\alpha_{i}-1\right) \mathbf{L} \prod_{j \in J}\left(\theta+\alpha_{j}-1\right)
$$

avec $I \cup J=\{1, \ldots, r\}$ et

$$
\mathbf{L}=L\left(\left\{\mu_{i}\right\}_{i \in I^{\prime}},\left\{\nu_{j}\right\}_{j \in J^{\prime}} ; p^{\prime}, q^{\prime}\right)
$$

un opérateur hypergéométrique Lie-irréductible;

- si $p^{\prime}=q^{\prime}-1$, le groupe $G^{s s}(L)$ est isomorphe au groupe $G\left(\alpha_{1}, \ldots, \alpha_{r}\right) \times$ $G^{L}$

- si $p^{\prime}<q^{\prime}-1$ et si $\left(G_{\mathbf{L}}\right)^{0, \mathrm{der}}=\mathrm{SL}_{q^{\prime}}(\mathbb{C})$, le groupe $G^{s s}(L)$ est isomorphe au sous-groupe de $G\left(\alpha_{1}, \ldots, \alpha_{r}\right) \times G_{\mathbf{L}}$ formés des $(r+1)$ uplets $\left\{a_{1}, \ldots, a_{r}, g\right\}$ tels que $\left\{a_{1}, \ldots, a_{r}, \operatorname{det}(g)\right\}$ appartienne à

$$
G\left(\alpha_{1}, \ldots, \alpha_{r}, \sum_{j \in J^{\prime}} \nu_{j}\right) .
$$

Dans tous les autres cas, le groupe $G^{s s}(L)$ est une extension finie du sousgroupe de $G\left(\alpha_{1}, \ldots, \alpha_{r}\right) \times G_{\mathbf{L}}$ formés des $(r+1)$-uplets $\left\{a_{1}, \ldots, a_{r}, g\right\}$ tels que $\left\{a_{1}, \ldots, a_{r}, \operatorname{det}(g)\right\}$ appartienne à $G\left(\alpha_{1}, \ldots, \alpha_{r}, \sum_{j \in J^{\prime}} \nu_{j}\right)$. 
Opérateurs hypergéométriques réductibles

2.3 Groupe de Galois sous les conditions $C(2,1,1)$

\subsection{1 Énoncés des résultats}

Si $m n=1$, alors $K^{u}(L)$ est nul. Le groupe $G^{u}(L)$ est isomorphe à $H^{u}(L)$.

Fixons alors les notations d'indices suivantes.

On choisit les couples d'indices $\left(i_{1}, j_{1}\right)$ et $\left(\mathbf{i}_{1}, \mathbf{j}_{1}\right)$ de telle sorte que :

$$
\begin{aligned}
& \mu_{i_{1}}-\nu_{j_{1}}=\inf \left\{\mu_{i}-\nu_{j} \mid \mu_{i}-\nu_{j} \in \mathbb{Z}, \mu_{i}-\nu_{j} \geq 0\right\} \\
& \mu_{\mathbf{i}_{1}}-\nu_{\mathbf{j}_{1}}=\inf \left\{\mu_{i}-\nu_{j} \mid \mu_{i}-\nu_{j} \in \mathbb{Z}, \mu_{i}-\nu_{j}<0\right\}
\end{aligned}
$$

Comme $m n=1$, d'après la définition de $m$ et $n$ et d'après le choix des indices, si $\nu_{j_{1}}-\nu_{\mathbf{j}_{1}} \in \mathbb{Z}$, on a :

$$
\Re \mu_{\mathbf{i}_{1}}<\Re \nu_{\mathbf{j}_{1}} \leq \Re \nu_{j_{1}} \leq \Re \mu_{i_{1}} \text { ou } \Re \nu_{\mathbf{j}_{1}}>\Re \mu_{\mathbf{i}_{1}} \geq \Re \mu_{i_{1}} \geq \Re \nu_{j_{1}} .
$$

D'après le lemme 1.2.2, on obtient facilement que l'opérateur $L$ est isomorphe à :

$$
\left(\theta+\nu_{j_{1}}-1\right) L\left(\left\{\mu_{i}\right\}_{i \notin\left\{i_{1}, \mathbf{i}_{1}\right\}},\left\{\nu_{j}\right\}_{j \notin\left\{j_{1}, \mathbf{j}_{1}\right\}} ; p-2, q-2\right)\left(\theta+\nu_{\mathbf{j}_{1}}-1\right) .
$$

Avec ces notations, on obtient le résultat suivant.

THÉORÈME 2.3.1. - Supposons que l'opérateur hypergéométrique

$$
L=L\left(\left\{\mu_{i}\right\}_{i},\left\{\nu_{j}\right\}_{j} ; p, q\right)
$$

vérifie les conditions $C(2,1,1)$ et que l'opérateur

$$
L\left(\left\{\mu_{i}\right\}_{i \in I^{\prime}},\left\{\nu_{j}\right\}_{j \in J^{\prime}} ; p-2, q-2\right)
$$

où $I^{\prime}=\left\{i \notin\left\{i_{1}, \mathbf{i}_{1}\right\}\right\}$ et $J^{\prime}=\left\{j \notin\left\{j_{1}, \mathbf{j}_{1}\right\}\right\}$ a un groupe de Galois Lieirréductible;

a) si, pour tout nombre complexe $\beta$, tel que $\nu_{j_{1}}+\nu_{\mathbf{j}_{1}}+2 \beta \in \mathbb{Z}$, l'opérateur

$$
L\left(\left\{\mu_{i}+\beta\right\}_{i \in I^{\prime}},\left\{\nu_{j}+\beta\right\}_{j \in J^{\prime}} ; p-2, q-2\right)
$$

$n^{\prime}$ est pas isomorphe à son dual, alors le groupe $G^{u}(L)$ est isomorphe $\grave{a} \mathbb{C}^{2 q-3}$; 
b) s'il existe un nombre complexe $\beta$, tel que $\nu_{j_{1}}+\nu_{\mathbf{j}_{1}}+2 \beta \in \mathbb{Z}$ et tel que le groupe de Galois de l'opérateur

$$
L\left(\left\{\mu_{i}+\beta\right\}_{i \in I^{\prime}},\left\{\nu_{j}+\beta\right\}_{j \in J^{\prime}} ; p-2, q-2\right)
$$

est un sous-groupe du groupe symplectique, alors le groupe $G^{u}(L)$ est isomorphe à $\mathbb{C}^{q-1}$;

c) s'il existe un nombre complexe $\beta$, tel que $\nu_{j_{1}}+\nu_{\mathrm{j}_{1}}+2 \beta \in \mathbb{Z}$ et tel que le groupe de Galois de l'opérateur

$$
L\left(\left\{\mu_{i}+\beta\right\}_{i \in I^{\prime}},\left\{\nu_{j}+\beta\right\}_{j \in J^{\prime}} ; p-2, q-2\right)
$$

est un sous-groupe du groupe orthogonal, alors le groupe de Galois $G^{u}(L)$ est isomorphe $\grave{a} \mathbb{C}^{q-2}$.

\subsubsection{Démonstration du théorème 2.3.1 a)}

Nous allons auparavant démontrer deux lemmes.

Lemme 2.3.1.- Soient $P, Q, R$ des opérateurs différentiels vérifiant les conditions suivantes :

a) les groupes $\mathrm{Gal}_{\text {diff }}\left(F_{P Q} / F_{P} F_{Q}\right)$ et $\mathrm{Gal}_{\text {diff }}\left(F_{Q R} / F_{Q} F_{R}\right)$ sont respectivement isomorphes aux groupes:

$$
\operatorname{Hom}\left(V_{P}, V_{Q}\right) \text { et } \operatorname{Hom}\left(V_{Q}, V_{R}\right) \text {; }
$$

b) les extensions $F_{P Q} F_{R}$ et $F_{Q R} F_{P}$ sont linéairement disjointes sur le corps $F_{P} F_{Q} F_{R}$.

Alors, le groupe $\left.\mathrm{Gal}_{\text {diff }}\left(F_{P Q R}\right) / F_{P} F_{Q} F_{R}\right)$ est isomorphe à :

$$
\operatorname{Hom}\left(V_{P}, V_{Q R}\right) \oplus \operatorname{Hom}\left(V_{Q}, V_{R}\right) \text {. }
$$

Démonstration. - Posons

$$
\left.H=\operatorname{Gal}_{\operatorname{diff}}\left(F_{P Q R}\right) / F_{P} F_{Q} F_{R}\right) .
$$

Supposons que les opérateurs $P, Q, R$ sont respectivement d'ordre $p, n, q$. 
Soit $\left\{f_{1}, \ldots, f_{p}, g_{1}, \ldots, g_{n}, h_{1}, \ldots, h_{q}\right\}$ une base de $V_{P Q R}$ telle que $\left\{h_{i}\right\}$ et $\left\{g_{1}, \ldots, g_{n}, h_{1}, \ldots, h_{q}\right\}$ soient des bases respectives de $V_{R}$ et $V_{Q R}$.

Dans cette base, les matrices des éléments de $H$ forment un groupe vérifiant les hypothèses du lemme A.1. En effet, en gardant les notations de ce lemme, les groupes

$$
\operatorname{Gal}_{\text {diff }}\left(F_{P Q} / F_{P} F_{Q}\right) \text { et } \operatorname{Gal}_{\text {diff }}\left(F_{Q R} / F_{Q} F_{R}\right)
$$

sont respectivement isomorphes aux groupes :

$$
\operatorname{Hom}\left(V_{P}, V_{Q}\right) \text { et } \operatorname{Hom}\left(V_{Q}, V_{R}\right) \text {; }
$$

les applications $\varphi$ de $G$ dans $M_{n, p}(\mathbb{C})$, qui à $M$ associe $u_{M}$, et $\psi$ de $G$ dans $M_{q, n}(\mathbb{C})$, qui à $M$ associe $v_{M}$, sont donc surjectives.

De plus, comme les extensions $F_{P Q} F_{R}$ et $F_{Q R} F_{P}$ sont linéairement disjointes sur le corps $F_{P} F_{Q} F_{R}$, d'après le lemme 2.1.2, les groupes $\mathrm{Gal}_{\text {diff }}\left(F_{P Q} F_{Q R} / F_{P} F_{Q R}\right)$ et $\mathrm{Gal}_{\text {diff }}\left(F_{P Q} / F_{P} F_{Q}\right)$ sont isomorphes, il en résulte que l'application $\varphi^{\prime}$ de $\left\{M \in H \mid v_{M}=0\right\}$ dans $M_{n, p}(\mathbb{C})$ qui à $M$ associe $u_{M}$ est surjective.

De la surjectivité des applications $\varphi^{\prime}$ et $\psi$, on déduit la surjectivité de l'application de $H$ dans $M_{n, p}(\mathbb{C}) \times M_{q, n}(\mathbb{C})$ qui à $M$ associe $\left(u_{M}, v_{M}\right)$.

Le groupe $H$ vérifie bien les hypothèses du lemme. On en déduit donc que $H$ est isomorphe à $\mathbb{C}^{(p+q) n+p q}$.

Le groupe $H$ est aussi isomorphe à

$$
\mathrm{Gal}_{\text {diff }}\left(F_{P Q R} / F_{P} F_{Q R}\right) \oplus \mathrm{Gal}_{\text {diff }}\left(F_{P} F_{Q R} / F_{P} F_{Q} F_{R}\right) .
$$

Par un raisonnement analogue à celui fait dans le lemme 2.2.2, nous obtenons un homomorphisme injectif de $H$ dans $\operatorname{Hom}\left(V_{P}, V_{Q R}\right) \oplus$ $\operatorname{Hom}\left(V_{Q}, V_{R}\right)$. Comme $H$ est isomorphe à $\mathbb{C}^{(p+q) n+p q}$, il est aussi isomorphe à :

$$
\operatorname{Hom}\left(V_{P}, V_{Q R}\right) \oplus \operatorname{Hom}\left(V_{Q}, V_{R}\right) \text {. }
$$

LEMmE 2.3.2.- Soit $L=P \mathbf{L} R$ un opérateur hypergéométrique avec $\mathbf{L}$ irréductible et soit $Q$ un opérateur quelconque. Pour un opérateur différentiel $S$, notons $S^{\prime}$ l'opérateur dont les solutions sont obtenues à partir des solutions de $S$ en les multipliant par $z^{-\beta}$. Alors les groupes

$$
\operatorname{Gal}_{\text {diff }}\left(F_{L} F_{Q} / F_{P} F_{\mathbf{L}} F_{R} F_{Q}\right) \text { et } \operatorname{Gal}_{\text {diff }}\left(F_{L^{\prime}} F_{Q^{\prime}} / F_{P^{\prime}} F_{\mathbf{L}^{\prime}} F_{R^{\prime}} F_{Q^{\prime}}\right)
$$

sont isomorphes et en particulier si $L$ vérifie les conditions $C(2,1,1)$, on a :

$$
H^{u}(L)=H^{u}\left(L^{\prime}\right) .
$$




\section{Katy Boussel}

Démonstration. - On a les relations évidentes

$$
F_{L^{\prime}}\left(z^{\beta}\right)=F_{L}\left(z^{\beta}\right) \quad \text { et } \quad F_{Q^{\prime}}\left(z^{\beta}\right)=F_{Q}\left(z^{\beta}\right) .
$$

Les extensions $F_{L^{\prime}} F_{Q^{\prime}}$ et $F_{P^{\prime}} F_{\mathbf{L}^{\prime}} F_{R^{\prime}} F_{Q^{\prime}}\left(z^{\beta}\right)$ sont linéairement disjointes sur le corps $F_{P^{\prime}} F_{\mathbf{L}^{\prime}} F_{R^{\prime}} F_{Q^{\prime}}$ puisque le groupe

$$
\operatorname{Gal}_{\operatorname{diff}}\left(F_{P^{\prime}} F_{\mathbf{L}^{\prime}} F_{R^{\prime}} F_{Q^{\prime}}\left(z^{\beta}\right) / F_{P^{\prime}} F_{\mathbf{L}^{\prime}} F_{R^{\prime}} F_{Q^{\prime}}\right)
$$

est un sous-groupe multiplicatif de $\mathbb{C}^{*}$ alors que le groupe

$$
\operatorname{Gal}_{\operatorname{diff}}\left(F_{L^{\prime}} F_{Q^{\prime}} / F_{P^{\prime}} F_{L^{\prime}} F_{R^{\prime}} F_{Q^{\prime}}\right)
$$

est un groupe additif.

Le groupe $\operatorname{Gal}_{\text {diff }}\left(F_{L^{\prime}} F_{Q^{\prime}}\left(z^{\beta}\right) / F_{P^{\prime}} F_{\mathbf{L}^{\prime}} F_{R^{\prime}} F_{Q^{\prime}}\left(z^{\beta}\right)\right)$ est donc isomorphe à $\operatorname{Gal}_{\text {diff }}\left(F_{L^{\prime}} F_{Q^{\prime}} / F_{P^{\prime}} F_{\mathbf{L}^{\prime}} F_{R^{\prime}} F_{Q^{\prime}}\right)$.

De même, les groupes

$$
\operatorname{Gal}_{\text {diff }}\left(F_{L} F_{Q}\left(z^{\beta}\right) / F_{P} F_{\mathbf{L}} F_{R} F_{Q}\left(z^{\beta}\right)\right) \text { et } \operatorname{Gal}_{\text {diff }}\left(F_{L} F_{Q} / F_{P} F_{\mathbf{L}} F_{R} F_{Q}\right)
$$

sont isomorphes. Comme le groupe $\operatorname{Gal}_{\text {diff }}\left(F_{L} F_{Q}\left(z^{\beta}\right) / F_{P} F_{\mathbf{L}} F_{R} F_{Q}\left(z^{\beta}\right)\right)$ est égal à $\operatorname{Gal}_{\text {diff }}\left(F_{L^{\prime}} F_{Q^{\prime}}\left(z^{\beta}\right) / F_{P^{\prime}} F_{\mathbf{L}^{\prime}} F_{R^{\prime}} F_{Q^{\prime}}\left(z^{\beta}\right)\right)$ d'après la relation (1), on en déduit donc bien la conclusion du lemme.

Suite de la démonstration du théorème 2.3.1 a)

L'opérateur $L=P \mathbf{L} R$ vérifiant les conditions $C(2,1,1)$, l'opérateur $P \mathbf{L}$ vérifie les conditions $C(1,1,0)$ et l'opérateur $\mathbf{L} R$ vérifie les conditions $C(1,0,1)$. D'après le théorème 2.2 .1 , les groupes

$$
\operatorname{Gal}_{\text {diff }}\left(F_{P \mathbf{L}} / F_{P} F_{\mathbf{L}}\right) \text { et } \operatorname{Gal}_{\operatorname{diff}}\left(F_{\mathbf{L} R} / F_{\mathbf{L}} F_{R}\right)
$$

sont respectivement isomorphes aux groupes $\operatorname{Hom}\left(V_{P}, V_{\mathbf{L}}\right)$ et $\operatorname{Hom}\left(V_{\mathbf{L}}, V_{R}\right)$. Il suffit de montrer que les extensions $F_{P \mathbf{L}} F_{R}$ et $F_{\mathbf{L} R} F_{P}$ sont linéairement disjointes sur le corps $F_{P} F_{\mathbf{L}} F_{R}$ et d'appliquer le lemme 2.3.1 pour avoir la conclusion de la proposition.

Supposons que les extensions $F_{P \mathbf{L}} F_{R}$ et $F_{\mathbf{L} R} F_{P}$ ne sont pas linéairement disjointes sur le corps $F_{P} F_{\mathbf{L}} F_{R}$. Nous allons montrer successivement les quatre points suivants : 
- les extensions $F_{P \mathbf{L}} F_{P} F_{R}$ et $F_{\mathbf{L} R} F_{P} F_{R}$ sont égales;

- il existe un nombre $\beta$ tel que l'opérateur

$$
\mathbf{L}^{\prime}=L\left(\left\{\mu_{i}+\beta\right\}_{i \in I^{\prime}},\left\{\nu_{j}+\beta\right\}_{j \in J^{\prime}} ; p-2, q-2\right)
$$

est isomorphe à son dual;

- le groupe $\operatorname{Gal}_{\text {diff }}\left(F_{P^{\prime} \mathbf{L}^{\prime}} F_{\mathbf{L}^{\prime} R^{\prime}} / F_{P^{\prime} \mathbf{L}^{\prime}} F_{R^{\prime}}\right)$ est nul, où $P^{\prime}=P+\beta$ et $R^{\prime}=R+\beta$;

- le nombre $\nu_{j_{1}}+\nu_{\mathbf{j}_{1}}+2 \beta \in \mathbb{Z}$.

La partie a) du théorème sera, alors, évidemment prouvée.

Montrons le premier point. Nous allons d'abord montrer que le groupe $M=\mathrm{Gal}_{\text {diff }}\left(F_{P \mathbf{L}} F_{\mathbf{L} R} / F_{P} F_{\mathbf{L} R}\right)$ est nul et qu'il en est de même du groupe $\mathrm{Gal}_{\text {diff }}\left(F_{P \mathbf{L}} F_{\mathbf{L} R} / F_{P \mathbf{L}} F_{R}\right)$.

Soit $\varphi$ une base de l'espace $V_{P}$, et soit $f$, un élément de $V_{P \mathbf{L}}$, vérifiant $\mathbf{L}(f)=\varphi$.

L'application $\xi_{f}$ qui, à tout $\sigma$ de $M$, associe $\sigma(f)-f$, définit un homomorphisme de $M$ dans $\operatorname{Hom}\left(V_{P}, V_{\mathbf{L}}\right)$ ou dans $V_{\mathbf{L}}$. L'application $\xi_{f}$ peut être prolongée au groupe $H=\operatorname{Gal}_{\text {diff }}\left(F_{P \mathbf{L}} F_{\mathbf{L} R} / F_{P} F_{R}\right)$. Le groupe $M$ est normal dans le groupe $H$.

Soit $\sigma^{\prime}$ un élément de $M$ et soit $\sigma$ un élément de $H$, on a :

$$
\xi_{f}\left(\sigma \sigma^{\prime} \sigma^{-1}\right)=\sigma \xi_{f}\left(\sigma^{\prime}\right) .
$$

Le groupe $K=\operatorname{Gal}_{\text {diff }}\left(F_{P \mathbf{L}} F_{\mathbf{L} R} / F_{P} F_{\mathbf{L}} F_{R}\right)$ étant abélien, $\xi_{f}\left(\sigma \sigma^{\prime} \sigma^{-1}\right)$ ne dépend que de la classe modulo $K$ de $\sigma$. Or $(H / K)$ est isomorphe à $\mathrm{Gal}_{\text {diff }}\left(F_{P} F_{\mathbf{L}} F_{R} / F_{P} F_{R}\right)$, notons $G_{\mathbf{L}}^{\prime}$, ce dernier groupe. Le groupe $M$ isomorphe à $\xi_{f}(M)$ peut donc être considéré comme un $G_{\mathbf{L}}^{\prime}$-module de $V_{\mathbf{L}}$.

D'après le lemme 2.2 .1 , le groupe $G_{\mathbf{L}}^{\prime}$ contient $\left(G_{\mathbf{L}}\right)^{0 \text {,der }}$ qui opère irréductiblement sur $V_{\mathbf{L}}$, donc, le groupe $M$, isomorphe à $\xi_{f}(M)$, ne peut différer de $V_{\mathbf{L}}$ que s'il est nul.

(1) Donc, si les extensions $F_{P \mathbf{L}} F_{R}$ et $F_{\mathbf{L} R} F_{P}$ ne sont pas linéairement disjointes sur le corps $F_{P} F_{\mathbf{L}} F_{R}$, alors le groupe $M$ est nul et par suite $F_{P \mathbf{L}} F_{R}$ est contenu dans $F_{\mathbf{L} R} F_{P}$.

(2) De même, alors, d'après le lemme 2.1.2, si les extensions $F_{P \mathbf{L}} F_{R}$ et $F_{\mathbf{L} R} F_{P}$ ne sont pas linéairement disjointes sur le corps $F_{P} F_{\mathbf{L}} F_{R}$, les groupes 
$\operatorname{Gal}_{\text {diff }}\left(F_{P \mathbf{L}} F_{\mathbf{L} R} / F_{P \mathbf{L}} F_{R}\right)$ et $\operatorname{Hom}\left(V_{\mathbf{L}}, V_{R}\right)$ sont distincts. Comme plus haut, le groupe $\operatorname{Gal}_{\text {diff }}\left(F_{P \mathbf{L}} F_{\mathbf{L} R} / F_{P \mathbf{L}} F_{R}\right)$ pouvant être considéré comme un $G_{\mathbf{L}^{-}}^{\prime}$ module de $V_{\mathbf{L}^{*}}$, s'il n'est pas isomorphe à $V_{\mathbf{L}^{*}}$, il est nul et par suite $F_{\mathbf{L} R} F_{R}$ est contenu dans $F_{P \mathbf{L}} F_{P}$.

De (1) et (2), on déduit que les extensions $F_{P \mathbf{L}} F_{P} F_{R}$ et $F_{\mathbf{L} R} F_{P} F_{R}$ sont égales. Le premier point est prouvé.

Démontrons le second point. Puisque les extensions $F_{P \mathbf{L}} F_{P} F_{R}$ et $F_{\mathbf{L} R} F_{P} F_{R}$ sont égales, les groupes

$$
\operatorname{Gal}_{\text {diff }}\left(F_{P \mathbf{L}} F_{P} F_{R} / F_{P} F_{\mathbf{L}} F_{R}\right) \text { et } \operatorname{Gal}_{\text {diff }}\left(F_{(\mathbf{L} R)^{*}} F_{P} F_{R} / F_{P} F_{\mathbf{L}} F_{R}\right)
$$

sont donc isomorphes en tant que $G_{\mathbf{L}}^{\prime}$-modules. Il existe donc un isomorphisme $A$ de $G_{\mathbf{L}}^{\prime}$-modules de $V_{\mathbf{L}}$ sur $V_{\mathbf{L}^{*}}$ donc sur le dual de $V_{\mathbf{L}}$. C'est-à-dire qu'il existe, dans une base donnée, un élément $A$ de $\mathrm{GL}_{q-2}(\mathbb{C})$ tel que pour tout élément $g$ de $G_{\mathbf{L}}^{\prime}$, on a ${ }^{t} g A g=A$.

Comme le groupe $G_{\mathbf{L}}^{\prime}$ opère irréductiblement sur $\mathbb{C}^{q-2}$, d'après le lemme A.2, l'isomorphisme $A$ est symétrique ou antisymétrique. Le groupe $G_{\mathbf{L}}^{\prime}$ serait un sous-groupe du groupe orthogonal ou symplectique. Le groupe $G_{\mathbf{L}}^{\prime}$ contenant le groupe $\left(G_{\mathbf{L}}^{\prime}\right)^{0, \text { der }}$, il en est de même pour le groupe $\left(G_{\mathbf{L}}\right)^{0, \mathrm{der}}$.

D'après le théorème de Katz (théorème 2.1.3), il existe un nombre $\beta$ tel que l'opérateur :

$$
\mathbf{L}^{\prime}=L\left(\left\{\mu_{i}+\beta\right\}_{i \in I^{\prime}},\left\{\nu_{j}+\beta\right\}_{j \in J^{\prime}} ; p-2, q-2\right)
$$

est isomorphe à son dual. Le deuxième point est prouvé.

Démontrons le troisième point. Considérons, alors, l'opérateur $L^{\prime}=$ $(P+\beta) \mathbf{L}^{\prime}(R+\beta)=P^{\prime} \mathbf{L}^{\prime} R^{\prime}$. Le groupe $\operatorname{Gal}_{\text {diff }}\left(F_{P \mathbf{L}} F_{\mathbf{L} R} / F_{P \mathbf{L}} F_{R}\right)$ est nul d'après le point 2. En appliquant le lemme 2.3.2 à $P=1$ et à $S=P \mathbf{L}$, on obtient alors que le groupe $\operatorname{Gal}_{\operatorname{diff}}\left(F_{P^{\prime} \mathbf{L}^{\prime}} F_{\mathbf{L}^{\prime} R^{\prime}} / F_{P^{\prime} \mathbf{L}^{\prime}} F_{R^{\prime}}\right)$ est nul aussi. Le troisième point est démontré.

Il nous reste à montrer le dernier point. Montrons que si $\nu_{j_{1}}+\nu_{\mathbf{j}_{1}}+2 \beta \notin \mathbb{Z}$, cela contredit la nullité du groupe $\operatorname{Gal}_{\text {diff }}\left(F_{P^{\prime} \mathbf{L}^{\prime}} F_{\mathbf{L}^{\prime} R^{\prime}} / F_{P^{\prime}} F_{\mathbf{L}^{\prime} R^{\prime}}\right)$.

Du théorème 1.2.2, on déduit que si l'opérateur $\mathbf{L}^{\prime}$ est isomorphe à son dual, alors il existe un opérateur $R^{\prime \prime *}$, isomorphe à $R^{\prime *}$, tel que l'opérateur $R^{\prime *} \mathbf{L}^{\prime *}$ est isomorphe à $R^{\prime \prime *} \mathbf{L}^{\prime}$. Posons

$$
P^{\prime}=\theta+\alpha, \quad R^{\prime}=\theta+\alpha^{\prime}, \quad R^{\prime \prime}=\theta+\alpha^{\prime \prime}
$$

avec $\alpha^{\prime}-\alpha^{\prime \prime} \in \mathbb{Z}$. 
Si $\alpha+\alpha^{\prime} \notin \mathbb{Z}$, les opérateurs $R^{\prime \prime *}$ et $P^{\prime}$ commutent et sont distincts, les extensions $F_{P^{\prime} \mathbf{L}^{\prime}} F_{R^{\prime \prime *} \mathbf{L}^{\prime}}$ et $F_{R^{\prime \prime *} P^{\prime} \mathbf{L}^{\prime}}$ sont donc égales sur le corps $K$. Les opérateurs $P^{\prime} \mathbf{L}^{\prime}$ et $R^{\prime \prime *} \mathbf{L}^{\prime}$ vérifiant les conditions $C(1,1,0)$ et $\alpha+\alpha^{\prime}$ n'appartenant pas à $\mathbb{Z}$, l'opérateur $R^{\prime \prime *} P^{\prime} \mathbf{L}^{\prime}$ vérifie les conditions $C(2,2,0)$, d'après la proposition 2.2.1, le groupe $\operatorname{Gal}_{\text {diff }}\left(F_{R^{\prime * *} P^{\prime} \mathbf{L}^{\prime}} / F_{P^{\prime} R^{\prime \prime *}} F_{\mathbf{L}^{\prime}}\right)$ est isomorphe à $\operatorname{Hom}\left(V_{R^{\prime \prime *} P^{\prime}}, V_{\mathbf{L}^{\prime}}\right)$. Ce qui contredit la nullité du groupe :

$$
\operatorname{Gal}_{\operatorname{diff}}\left(F_{P^{\prime} \mathbf{L}^{\prime}} F_{\mathbf{L}^{\prime} R^{\prime}} F_{R^{\prime}} / F_{P^{\prime} \mathbf{L}^{\prime}} F_{R^{\prime}}\right) \text {. }
$$

Donc, on a $\alpha+\alpha^{\prime} \in \mathbb{Z}$, ce qui équivaut à $\nu_{j_{1}}+\nu_{\mathbf{j}_{1}}+2 \beta \in \mathbb{Z}$. Le dernier point est prouvé.

\subsubsection{Démonstration du théorème 2.3.1 b)}

Montrons d'abord un lemme.

Lemme 2.3.3. - Soit $L=(\theta+\alpha) \mathbf{L}(\theta+\beta)$ un opérateur vérifiant les conditions $C(2,1,1)$. L'opérateur $L$ est isomorphe à son dual si et seulement si $\alpha+\beta$ est un entier et $\mathbf{L}$ est isomorphe à son dual.

Démonstration. - Considérons l'opérateur dual de L. On a :

$$
L^{*}=(\theta-\beta) \mathbf{L}^{*}(\theta-\alpha) .
$$

Si $\alpha$ et $\beta$ sont congrus modulo $Z$, les différences $\beta-\alpha$ et $(-\alpha)-(-\beta)$ sont nécessairement de même signe puisqu'elles sont égales. Il suffit alors d'appliquer le théorème 1.2.2 pour avoir la conclusion du lemme.

Suite de la démonstration du théorème 2.3.1 b)

Considérons l'opérateur

$$
L^{\prime}=(P+\beta) \mathbf{L}^{\prime}(R+\beta)=P^{\prime} \mathbf{L}^{\prime} R^{\prime},
$$

avec

$$
\mathbf{L}^{\prime}=L\left(\left\{\mu_{i}+\beta\right\}_{i \in I^{\prime}}\left\{\nu_{j}+\beta\right\}_{j \in J^{\prime}} ; p-2, q-2\right) .
$$

D'après le lemme 2.3.2, les groupes $H^{u}(L)$ et $H^{u}\left(L^{\prime}\right)$ sont isomorphes. Montrons que le groupe

$$
G=H^{u}\left(L^{\prime}\right)=\operatorname{Gal}_{\operatorname{diff}}\left(F_{P^{\prime} \mathbf{L}^{\prime} R^{\prime}} / F_{P^{\prime}} F_{R^{\prime}}\right)
$$

vérifie les hypothèses du lemme A.3bis. Choisissons une base dans laquelle les matrices des éléments de $G$ ont la forme de la matrice $M$ de ce même lemme. 
- Montrons qu'il existe un élément $A$ de $\mathrm{GL}_{q}(\mathbb{C})$ tel que pour tout $g \in \mathrm{Gal}_{\text {diff }}\left(F_{P^{\prime} \mathbf{L}^{\prime} R^{\prime}} / F_{P^{\prime}} F_{R^{\prime}}\right),{ }^{t} g A g=A$.

D'après le lemme 2.3.3, l'opérateur $L^{\prime}$ est isomorphe à son dual. Il en résulte, puisque le groupe $\operatorname{Gal}_{\text {diff }}\left(F_{P^{\prime} \mathbf{L}^{\prime} R^{\prime}} / F_{P^{\prime}} F_{R^{\prime}}\right)$ est un sous-groupe de $G_{L^{\prime}}$, qu'il existe un élément $A$ de $\mathrm{GL}_{q}(\mathbb{C})$ tel que pour tout $g \in$ $\mathrm{Gal}_{\text {diff }}\left(F_{P^{\prime} \mathbf{L}^{\prime} R^{\prime}} / F_{P^{\prime}} F_{R^{\prime}}\right),{ }^{t} g A g=A$.

- Le groupe $H=\operatorname{Gal}_{\text {diff }}\left(F_{\mathbf{L}^{\prime}} F_{P^{\prime}} F_{R^{\prime}} / F_{P^{\prime}} F_{R^{\prime}}\right)$ est un sous-groupe du groupe symplectique, par hypothèse qui, d'après le lemme 2.2.1, opère irréductiblement sur $\mathbb{C}^{q-2}$.

- Les groupes

$$
\operatorname{Gal}_{\text {diff }}\left(F_{P^{\prime} \mathbf{L}^{\prime}} F_{R^{\prime}} / F_{P^{\prime}} F_{R^{\prime}}\right) \text { et } \operatorname{Gal}_{\text {diff }}\left(F_{P} F_{\mathbf{L}^{\prime} R^{\prime}} / F_{P^{\prime}} F_{R^{\prime}}\right)
$$

sont, d'après la proposition 2.2.1 et le lemme 2.3.1, des extensions de $H=\operatorname{Gal}_{\text {diff }}\left(F_{\mathbf{L}^{\prime}} F_{P^{\prime}} F_{R^{\prime}} / F_{P^{\prime}} F_{R^{\prime}}\right)$ respectivement par $\operatorname{Hom}\left(V_{P^{\prime}}, V_{\mathbf{L}^{\prime}}\right)$ et par $\operatorname{Hom}\left(V_{\mathbf{L}}, V_{P^{\prime}}\right)$. On en déduit ainsi la surjectivité des deux applications mentionnées dans l'hypothèse du lemme A.3bis.

Le groupe $H^{u}\left(L^{\prime}\right)=\operatorname{Gal}_{\mathrm{diff}}\left(F_{P^{\prime} \mathbf{L}^{\prime} R^{\prime}} / F_{P^{\prime}} F_{R^{\prime}}\right)$ vérifie donc les hypothèses du lemme A.3bis. Le groupe $H^{u}\left(L^{\prime}\right)$ est ainsi isomorphe à $\mathbb{C}^{q-1}$. De l'isomorphisme des groupes $H^{u}\left(L^{\prime}\right)$ et $H^{u}(L)$, on en déduit la proposition.

Remarque. - Les opérateurs $P \mathbf{L}$ et $\mathbf{L} R$ vérifiant respectivement les conditions $C(1,1,0)$ et $C(1,0,1)$, d'après les hypothèses a) et b) de la proposition, on déduit du théorème 1.2.2, l'équivalence des opérateurs $P^{\prime} \mathbf{L}^{\prime}$ et $\left(\mathbf{L}^{\prime} R^{\prime}\right)^{*}$. Le groupe $\mathrm{Gal}_{\text {diff }}\left(F_{P^{\prime} \mathbf{L}^{\prime}} F_{R^{\prime} \mathbf{L}^{\prime}} / F_{P^{\prime}} F_{\mathbf{L}^{\prime} R^{\prime}}\right)$ est donc nul.

On obtient, comme dans la démonstration du lemme 2.2.2 un homomorphisme injectif de $H^{u}\left(L^{\prime}\right)$ dans $\operatorname{Hom}\left(V_{P^{\prime}}, V_{\mathbf{L}^{\prime} R^{\prime}}\right)$. Le groupe $H^{u}\left(L^{\prime}\right)$ étant isomorphe à $\mathbb{C}^{q-1}$, il est aussi isomorphe à $\operatorname{Hom}\left(V_{P^{\prime}}, V_{\mathbf{L}^{\prime} R^{\prime}}\right)$.

\subsubsection{Démonstration du théorème 2.3.1 c)}

La démonstration est pratiquement identique à la démonstration précédente, à part que, ici, le groupe $\mathrm{Gal}_{\text {diff }}\left(F_{\mathbf{L}^{\prime}} F_{P^{\prime}} F_{R^{\prime}} / F_{P^{\prime}} F_{R^{\prime}}\right)$ est un sousgroupe du groupe orthogonal qui, d'après le lemme 2.2.1, opère irréductiblement sur $\mathbb{C}^{q-2}$ et que le groupe $\operatorname{Gal}_{\text {diff }}\left(F_{P^{\prime} \mathbf{L}^{\prime} R^{\prime}} / F_{P^{\prime}} F_{R^{\prime}}\right)$ vérifie les hypothèses du lemme A.4. Le groupe $H^{u}\left(L^{\prime}\right)$ est donc isomorphe à $\mathbb{C}^{q-2}$. Le groupe $H^{u}\left(L^{\prime}\right)$ est aussi isomorphe à $\operatorname{Hom}\left(V_{P^{\prime}}, V_{\mathbf{L}^{\prime}}\right)$. Comme précédemment, de l'isomorphisme des groupes $H^{u}\left(L^{\prime}\right)$ et $H^{u}(L)$ on en déduit la proposition. 
Signalons pour finir que la situation dans le cas $C(m+n, m, n)$ avec $m n>1$ est plus complexe car on n'a pas l'analogue du lemme 2.3.3. Supposons que l'opérateur $L$ soit isomorphe à $\prod_{i \in I} P_{i} L^{\prime} \prod_{j \in J} P_{j}$ avec $L^{\prime}$ opérateur hypergéométrique irréductible. Le groupe de Galois de $L$ peut ne pas être aussi gros que possible sans que l'opérateur $L$ soit forcément isomorphe à son dual. On obtient simplement que l'extension de PicardVessiot de $L^{\prime}$ est égale à l'extension de Picard-Vessiot d'un opérateur isomorphe à son dual auquel on a adjoint éventuellement $\log z$.

Par contre, le cas "dégénéré" se ramène facilement au cas "non dégénéré" d'un opérateur d'ordre inférieur grâce à des considérations sur l'extension de Picard-Vessiot de l'opérateur.

\section{Appendice}

Lemme A.1. - Soit $G$ un sous-groupe de $\mathrm{GL}_{n+p+q}(\mathbb{C})$ contenant des matrices de la forme:

$$
M=\left(\begin{array}{ccc}
\operatorname{Id}_{p} & 0 & 0 \\
u_{M} & \operatorname{Id}_{n} & 0 \\
w_{M} & v_{M} & \operatorname{Id}_{q}
\end{array}\right)
$$

avec $u_{M} \in M_{n, p}(\mathbb{C}), v_{M} \in M_{q, n}(\mathbb{C})$ et $w_{M} \in M_{q, p}(\mathbb{C})$.

Si l'application de $G$ dans $M_{n, p}(\mathbb{C}) \times M_{q, n}(\mathbb{C})$ qui à $M$ associe $\left(u_{M}, v_{M}\right)$ est surjective, alors l'application de $G$ dans $M_{n, p}(\mathbb{C}) \times M_{q, n}(\mathbb{C}) \times M_{q, p}(\mathbb{C})$ qui à $M$ associe $\left(u_{M}, v_{M}, w_{M}\right)$ est surjective.

Démonstration. - On a les relations :

$$
u_{M M^{\prime} M^{-1}}=u_{M^{\prime}} \quad v_{M M^{\prime} M^{-1}}=v_{M^{\prime}}
$$

et

$$
w_{M M^{\prime} M^{-1}}=w_{M^{\prime}}+v_{M} u_{M^{\prime}}-v_{M^{\prime}} u_{M} .
$$

Il existe un élément $M^{\prime}$ de $G$ tel que la dimension de l'espace vectoriel $E=\left\{v_{M} u_{M^{\prime}}-v_{M^{\prime}} u_{M} \mid M \in G\right\}$ est égale à $\operatorname{Dim}_{\mathbb{C}} M_{q, p}(\mathbb{C})=q p$.

En effet, il suffit de choisir pour matrice $M^{\prime}$, une matrice telle que $v_{M^{\prime}}=0$ et telle que $u_{M^{\prime}}$ a exactement $\inf (q, n)$ éléments non nuls et de telle sorte que chaque colonne et chaque ligne aient au plus un élément non nul. En 
prenant tous les éléments $M$ de $G$ tels que $v_{M}$ soit un multiple d'une matrice élémentaire, il est facile de voir que toutes les multiples des matrices élémentaires sont dans $E$.

Donc, si $M^{\prime}$ est la matrice définie ci-dessus, la dimension de l'espace vectoriel $E$ est supérieure ou égale à $p q$ et, par suite, égale à $p q$. Ce qui implique que la dimension de l'espace vectoriel

$$
\left\{w_{M} \mid M \in G, u_{M}=u_{M} \text { et } v_{M}=v_{M^{\prime}}\right\}
$$

est aussi égale à $p q$. On en déduit que :

$$
\operatorname{Dim}_{\mathbb{C}}\left\{\left(u_{M}, v_{M}, w_{M}\right) \mid M \in G\right\}=n(p+q)+q p .
$$

Ce qui suffit à prouver le lemme.

Lemme A.2. - Soit $H$ un sous-groupe de $\mathrm{GL}_{n}(\mathbb{C})$, opérant irréductiblement sur $\mathbb{C}^{n}$. Supposons qu'il existe un élément $A$ de $M_{n, n}(\mathbb{C})$ tel que pour tout élément $g$ de $H,{ }^{t} g A g=A$. Alors :

i) si $A^{\prime}$ est un élément de $M_{n, n}(\mathbb{C})$ tel que pour tout $g$ de $H,{ }^{t} g A^{\prime} g=A^{\prime}$, alors il existe un couple de $\mathbb{C}^{2},(\lambda, \mu) \neq(0,0)$, tels que $\lambda A+\mu A^{\prime}=0 ;$

ii) la matrice $A$ est symétrique ou antisymétrique.

Démonstration. - Remarquons que si $A$ est un endomorphisme dégénéré de $\mathbb{C}^{n}$ vérifiant pour tout élément $g$ de $H,{ }^{t} g A g=A$, alors $A$ est nul.

En effet, pour tout élément $g$ de $H,{ }^{t} g A=A g^{-1}$. Il en résulte que le noyau de $A$ est stable sous $H$. Or, $H$ opérant irréductiblement sur $\mathbb{C}^{n}$, on en déduit que le noyau de $A$ est soit nul, soit égal à $\mathbb{C}^{n}$. Si $A$ est dégénéré, son noyau n'est pas nul, il est donc égal à $\mathbb{C}^{n}$, ce qui prouve que $A$ est nul. i) $\mathrm{Si} A$ ou $A^{\prime}$ est nul, l'assertion est évidente. Supposons, donc $A$ et $A^{\prime}$ non nuls. Il existe $\lambda \neq 0$ tel que $A^{\prime}-\lambda A$ est dégénéré. $\mathrm{D}$ 'après la remarque, $A^{\prime}-\lambda A$ est nul.

ii) On peut supposer que $A$ est un endomorphisme non nul de $\mathbb{C}^{n}$. La matrice ${ }^{t} A+A=A^{\prime}$ vérifie, pour tout élément $g$ de $H,{ }^{t} g A^{\prime} g=A^{\prime}$. Si ${ }^{t} A+A$ n'est pas nul, d'après i), il existe une constante $\lambda \neq 0$ telle que $A=\lambda\left({ }^{t} A+A\right)$. La matrice $A$ est donc symétrique. Si ${ }^{t} A+A$ est nul, alors $A$ est une matrice antisymétrique.

On a bien montré que $A$ est symétrique ou antisymétrique. 
Lemme A.3. - Soit $H$ un sous-groupe de $\mathrm{GL}_{2 n}(\mathbb{C})$ et soit $G$ un sousgroupe de $\mathrm{GL}_{2 n+2}(\mathbb{C})$ contenant des matrices de la forme :

$$
M=\left(\begin{array}{ccc}
1 & 0 & 0 \\
u_{M} & \sigma_{M} & 0 \\
w_{M} & { }^{t} u_{M} J \sigma_{M} & 1
\end{array}\right)
$$

où $u_{M} \in M_{2 n, 1}(\mathbb{C}), \sigma_{M} \in H$ et $w_{M} \in \mathbb{C}$, avec

$$
J=\left(\begin{array}{cc}
0 & \operatorname{Id}_{n} \\
-\operatorname{Id}_{n} & 0
\end{array}\right)
$$

Si $H$ contient $J$ et si l'application de $G$ dans $M_{2 n, 1}(\mathbb{C}) \times H$, qui à $M$ associe $\left(u_{M}, \sigma_{M}\right)$, est surjective alors $G$ contient le groupe :

$$
\left\{\left(\begin{array}{ccc}
1 & 0 & 0 \\
u & \sigma & 0 \\
w & { }^{t} u J \sigma & 1
\end{array}\right) \text { où } u \in M_{2 n, 1}(\mathbb{C}), \sigma \in H \text { et } w \in \mathbb{C}\right\} .
$$

Démonstration. - Montrons que $w$ ne peut pas être une fonction algébrique de $u$ et $\sigma$. Posons $w=\varphi(u, \sigma)$. Du calcul du produit des matrices :

$$
\begin{aligned}
& \left(\begin{array}{ccc}
1 & 0 & 0 \\
u & \sigma & 0 \\
w & { }^{t} u J \sigma & 1
\end{array}\right)\left(\begin{array}{ccc}
1 & 0 & 0 \\
u^{\prime} & \sigma^{\prime} & 0 \\
w^{\prime} & { }^{t} u^{\prime} J \sigma^{\prime} & 1
\end{array}\right)= \\
& \quad=\left(\begin{array}{ccc}
1 & 0 & 0 \\
u+\sigma u^{\prime} & \sigma \sigma^{\prime} & 0 \\
w+{ }^{t} u J \sigma u^{\prime}+w^{\prime} & { }^{t} u J \sigma \sigma^{\prime}+{ }^{t} u^{\prime} J \sigma^{\prime} & 1
\end{array}\right)
\end{aligned}
$$

on obtient la relation, pour tout $u, u^{\prime}$ de $M_{2 n, 1}(\mathbb{C}), \sigma$ et $\sigma^{\prime}$ éléments de $\left\{\operatorname{Id}_{2 n},-\operatorname{Id}_{2 n}, J\right\}$,

$$
\varphi\left(u+\sigma u^{\prime}, \sigma \sigma^{\prime}\right)=\varphi(u, \sigma)+{ }^{t} u J \sigma u^{\prime}+\varphi\left(u^{\prime}, \sigma^{\prime}\right) .
$$

En prenant $u=u^{\prime}=0, \sigma=\sigma^{\prime}=\operatorname{Id}_{2 n}$, puis $u=u^{\prime}=0, \sigma=\sigma^{\prime}=-\operatorname{Id}_{2 n}$ et enfin $u=u^{\prime}=0, \sigma=\sigma^{\prime}=J$, on obtient successivement les relations :

$$
\varphi\left(0, \operatorname{Id}_{2 n}\right)=0, \quad \varphi\left(0,-\operatorname{Id}_{2 n}\right)=0, \quad \varphi(0, J)=0 .
$$


En prenant $u=u^{\prime}, \sigma=\sigma^{\prime}=-\operatorname{Id}_{2 n}$, on obtient :

$$
\varphi\left(0, \operatorname{Id}_{2 n}\right)=\varphi\left(u,-\operatorname{Id}_{2 n}\right)-{ }^{t} u J u+\varphi\left(u,-\operatorname{Id}_{2 n}\right),
$$

d'où

$$
\varphi\left(u,-\operatorname{Id}_{2 n}\right)=\frac{{ }^{t} u J u}{2},
$$

l'élément ${ }^{t} u J u$ appartenant à $\mathbb{C}$, on a ${ }^{t}\left({ }^{t} u J u\right)={ }^{t} u J u$, mais, aussi ${ }^{t}\left({ }^{t} u J u\right)={ }^{t} u^{t} J u=-{ }^{t} u J u$ puisque $J$ est une matrice antisymétrique. Donc $\varphi\left(u,-\operatorname{Id}_{2 n}\right)=0$ pour tout $u$ de $M_{2 n, 1}(\mathbb{C})$.

En appliquant la relation (1) d'abord à $u^{\prime}=0, \sigma=\sigma^{\prime}=-\operatorname{Id}_{2 n}$ puis à $u^{\prime}=0, \sigma=\sigma^{\prime}=J$, on obtient successivement :

$$
\varphi\left(u, \operatorname{Id}_{2 n}\right)=0, \quad \varphi(u, J)=0
$$

pour tout $u$ de $M_{2 n, 1}(\mathbb{C})$.

Finalement, la relation (1) appliquée à $u=u^{\prime}, \sigma=J, \sigma^{\prime}=\operatorname{Id}_{2 n}$, donne :

$$
\varphi(u+J u, J)=\varphi(u, J)+{ }^{t} u J^{2} u+\varphi\left(u, \operatorname{Id}_{2 n}\right)
$$

soit ${ }^{t} u J^{2} u=0$ pour tout $u$ de $M_{2 n, 1}(\mathbb{C})$. Or comme $J^{2}=-\operatorname{Id}_{2 n}$, on devrait avoir, pour tout $u$ de $M_{2 n, 1}(\mathbb{C}),-{ }^{t} u u=0$. Ce qui est, naturellement, impossible, d'où le résultat du lemme.

Remarque. - Il est facile de voir qu'au moyen d'un changement de base, on obtient le résultat suivant.

Soit $H$ un sous-groupe de $\mathrm{GL}_{2 n}(\mathbb{C})$, soit $\alpha \in \mathrm{GL}_{2 n}(\mathbb{C})$ et soit $G$ un sous-groupe de $\mathrm{GL}_{2 n+2}(\mathbb{C})$ contenant des matrices de la forme :

$$
M=\left(\begin{array}{ccc}
1 & 0 & 0 \\
u_{M} & \sigma_{M} & 0 \\
w_{M} & \alpha\left(1-\sigma_{M}\right)+{ }^{t} u_{M} J \sigma_{M} & 1
\end{array}\right)
$$

où $u_{M} \in M_{2 n, 1}(\mathbb{C}), \sigma_{M} \in H$ et $w_{M} \in \mathbb{C}$. Si $H$ contient $J$ et si l'application de $G$ dans $M_{2 n, 1}(\mathbb{C}) \times H$, qui à $M$ associe $\left(u_{M}, \sigma_{M}\right)$ est surjective, alors $G$ contient le groupe :

$$
\left\{\left(\begin{array}{ccc}
1 & 0 & 0 \\
u & \sigma & 0 \\
w & \alpha(1-\sigma)+{ }^{t} u J \sigma & 1
\end{array}\right) \text { où } u \in M_{2 n, 1}(\mathbb{C}), \sigma \in H \text { et } w \in \mathbb{C}\right\} .
$$

Dans le même ordre d'idée, on obtient le résultat suivant. 
LEMME A.3bis. - Soit $H$ un sous-groupe du groupe symplectique $\operatorname{Sp}_{2 n}(\mathbb{C})$ opérant irréductiblement sur $\mathbb{C}^{2 n}$ et soit $G$ un sous-groupe de $\mathrm{GL}_{2 n+2}(\mathbb{C})$ tel qu'il existe un élément $A$ de $\mathrm{GL}_{2 n+2}(\mathbb{C})$ vérifiant ${ }^{t} M A M=A$ pour tout $M$ de $G$ et dont les éléments sont de la forme:

$$
M=\left(\begin{array}{ccc}
1 & 0 & 0 \\
u_{M} & \sigma_{M} & 0 \\
x_{M} & { }^{t} v_{M} & 1
\end{array}\right)
$$

avec $u_{M}, v_{M} \in M_{2 n, 1}(\mathbb{C}), \sigma_{M} \in H$ et $x_{M} \in \mathbb{C}$.

Supposons que l'application de $G$ dans $M_{2 n, 1}(\mathbb{C}) \times H$ qui à $M$ associe $\left(u_{M}, \sigma_{M}\right)$ et l'application de $G$ dans $M_{2 n, 1}(\mathbb{C}) \times H$ qui à $M$ associe $\left(v_{M}, \sigma_{M}\right)$ sont surjectives. Alors :

a) il existe des constantes $a, c \neq 0$ et $\lambda \neq 0$, un élément $b$ de $M_{2 n, 1}(\mathbb{C})$ tels que :

$$
A=\left(\begin{array}{ccc}
a & -{ }^{t} b & -c \\
b & \lambda J & 0 \\
c & 0 & 0
\end{array}\right)
$$

avec

$$
J=\left(\begin{array}{cc}
0 & \operatorname{Id}_{n} \\
-\operatorname{Id}_{n} & 0
\end{array}\right)
$$

b) si $C$ désigne la constante $\lambda / c$ et $B$ l'élément $(1 / c)^{t} b$ de $M_{2 n, 1}(\mathbb{C})$, alors

$$
\left\{\left(\begin{array}{ccc}
1 & 0 & 0 \\
u & \sigma & 0 \\
x & B(1-\sigma)+C^{t} u J \sigma & 1
\end{array}\right) \text { où } u \in M_{n, 1}(\mathbb{C}), \sigma \in H \text { et } x \in \mathbb{C}\right\}
$$

et le sous-groupe $G^{\prime}$ formé des éléments $M$ de $G$ tels que $\sigma_{M}=\operatorname{Id}_{2 n}$ est de dimension $2 n+1$.

Remarque. - Par un changement de base, on se ramène au cas où $C=1$ et $B=0$.

Démonstration. - Posons :

$$
\begin{aligned}
A= & \left(\begin{array}{lll}
a & a^{\prime} & a^{\prime \prime} \\
b & b^{\prime} & b^{\prime \prime} \\
c & c^{\prime} & c^{\prime \prime}
\end{array}\right) \\
& -357-
\end{aligned}
$$




\section{Katy Boussel}

avec $a, a^{\prime \prime}, c, c^{\prime \prime} \in \mathbb{C}, b^{\prime} \in M_{2 n, 2 n}(\mathbb{C}), b, b^{\prime \prime} \in M_{2 n, 1}(\mathbb{C}), a^{\prime}, c^{\prime} \in M_{1,2 n}(\mathbb{C})$. En écrivant : ${ }^{t} M A M=A$ pour tout $M$ appartenant à $G$, on obtient les relations :

- ${ }^{t} \sigma b^{\prime \prime}+v c^{\prime \prime}=b^{\prime \prime}$ pour tout $\sigma \in H$ et $v \in M_{2 n, 1}(\mathbb{C})$, d'où $b^{\prime \prime}=0$ et $c^{\prime \prime}=0$

- $c^{\prime} \sigma=c^{\prime}$ pour tout $\sigma \in H$, d'où $c^{\prime}=0$;

- ${ }^{t} \sigma b^{\prime} \sigma=b^{\prime}$ pour tout $\sigma \in H$; d'après le lemme A.2, comme $H$ opère irréductiblement sur $\mathbb{C}^{2 n}$, il existe une constante $\lambda \neq 0$ telle que $b^{\prime}=\lambda J$

- $a^{\prime} \sigma+{ }^{t} u b^{\prime} \sigma+a^{\prime \prime t} v=a^{\prime} \quad$ pour tout $\sigma \in H$ et $v \in M_{1,2 n}(\mathbb{C})$;

- ${ }^{t} \sigma b+v c+{ }^{t} \sigma b^{\prime} u=b$ pour tout $\sigma \in H$ et $v \in M_{1,2 n}(\mathbb{C})$; en transposant cette relation, comme ${ }^{t} b^{\prime}=-b^{\prime}$ puisque $b^{\prime}=\lambda J$, on obtient :

$$
{ }^{t} b \sigma-{ }^{t} u b^{\prime} \sigma+{ }^{t} c^{t} v={ }^{t} b \quad \text { pour tout } \sigma \in H \text { et } v \in M_{1,2 n}(\mathbb{C}) \text {; }
$$

en additionnant les relations (1) et (2), on a $\left({ }^{t} b+a^{\prime}\right) \sigma+\left({ }^{t} c+a^{\prime \prime}\right){ }^{t} v=$ ${ }^{t} b+a^{\prime}$ pour tout $\sigma \in H$ et $v \in M_{1,2 n}(\mathbb{C})$; on en déduit :

$$
a^{\prime \prime}=-{ }^{t} c=-c \text { et } \quad a^{\prime}=-{ }^{t} b ;
$$

- $a+{ }^{t} u b^{\prime} u+{ }^{t} u b+x c+a^{\prime} u+a^{\prime \prime} x=a$ pour tout $u \in M_{2 n, 1}(\mathbb{C})$.

Cette dernière relation est toujours vérifiée car, comme ${ }^{t} b^{\prime}=-b^{\prime}$, on a

$$
\left({ }^{t} u b^{\prime} u\right)^{t}=-{ }^{t} u b^{\prime} u .
$$

Mais ${ }^{t} u b^{\prime} u$ étant un élément de $\mathbb{C}$, on a aussi :

$$
\left({ }^{t} u b^{\prime} u\right)^{t}={ }^{t} u b^{\prime} u .
$$

Des relations (4) et (5), on déduit que ${ }^{t} u b^{\prime} u=0$.

Comme $a^{\prime} u+a^{\prime \prime} x$ appartient à $\mathbb{C}$, il est égal à son transposé :

$$
a^{\prime} u+a^{\prime \prime} x={ }^{t} u^{t} a^{\prime}+{ }^{t} x{ }^{t} a^{\prime \prime}=-{ }^{t} u b-{ }^{t} x c,
$$

d'après les relations (3). La dernière relation devient $a=a$. a) est ainsi démontré. 
Démontrons $\mathrm{b}$ ). Comme $A$ n'est pas dégénérée, $c$ est une constante non nulle et si l'on pose $C=\lambda / c$ et $B=(1 / c)^{t} b$, alors :

$$
{ }^{t} v=B(1-\sigma)+C^{t} u J \sigma .
$$

En multipliant le dernier vecteur de la base de $\mathrm{GL}_{2 n+2}(\mathbb{C})$ par $(1 / k)$, on obtient une nouvelle base dans laquelle le groupe $G$ vérifie les hypothèses du groupe cité dans la remarque qui précède l'énoncé de ce lemme. De cette remarque et de la relation (6), on obtient bien le b) du lemme A.3bis.

LEMME A.4. - Soit $H$ un sous-groupe du groupe orthogonal $\mathrm{O}_{n}(\mathbb{C})$ opérant irréductiblement sur $\mathbb{C}^{n}$ et soit $G$ un sous-groupe de $\mathrm{GL}_{n+2}(\mathbb{C})$ tel qu'il existe une matrice $A$ de $\mathrm{GL}_{n+2}(\mathbb{C})$ vérifiant ${ }^{t} M A M=A$ pour tout $M$ de $G$ et dont les éléments sont de la forme:

$$
M=\left(\begin{array}{ccc}
1 & 0 & 0 \\
u_{M} & \sigma_{M} & 0 \\
x_{M} & { }^{t} v_{M} & 1
\end{array}\right)
$$

avec $u_{M}, v_{M} \in M_{n, 1}(\mathbb{C})$ et $\sigma_{M} \in H$.

Supposons que l'application de $G$ dans $M_{n, 1}(\mathbb{C}) \times H$ qui à $M$ associe $\left(u_{M}, \sigma_{M}\right)$ et l'application de $G$ dans $M_{n, 1}(\mathbb{C}) \times H$ qui à $M$ associe $\left(v_{M}, \sigma_{M}\right)$ sont surjectives. Alors :

a) il existe des constantes $a, c \neq 0$, et $\lambda \neq 0$, un élément $b$ de $M_{n, 1}(\mathbb{C})$ tels que

$$
A=\left(\begin{array}{ccc}
a & { }^{t} b & c \\
b & \lambda \operatorname{Id}_{n} & 0 \\
c & 0 & 0
\end{array}\right)
$$

b) si $C$ désigne la constante $-\lambda / c$ et $B$ l'élément $(-1 / c)^{t} b$ de $M_{n, 1}(\mathbb{C})$, alors

$$
G=\left\{\left(\begin{array}{ccc}
1 & 0 & 0 \\
u & \sigma & 0 \\
B u+\frac{C^{t} u u}{2} & B(\sigma-1)+C^{t} u \sigma & 1
\end{array}\right) \text { ò̀ } u \in M_{n, 1}(\mathbb{C}), \sigma \in H\right\}
$$

et le sous-groupe $G^{\prime}$ formé des éléments $M$ de $G$ tels que $\sigma_{M}=\operatorname{Id}_{n}$ est de dimension $n$. 
Remarque A.1. - Dans une base convenable, on a :

$$
G=\left\{\left(\begin{array}{ccc}
1 & 0 & 0 \\
u & \sigma & 0 \\
\frac{{ }^{t} u u}{2} & { }^{t} u \sigma & 1
\end{array}\right) \text { où } u \in M_{n, 1}(\mathbb{C}), \sigma \in H\right\} .
$$

Remarque A.2. - Soit :

$$
G=\left\{\left(\begin{array}{ccc}
\lambda & 0 & 0 \\
u & \sigma & 0 \\
{ }^{-1}{ }^{t} \frac{u u}{2} & { }^{t} u \sigma k \lambda^{-1} & \lambda^{-1}
\end{array}\right) \quad \begin{array}{c}
\text { où } \lambda \in K, k \in \mathbb{C}^{*}, \\
\sigma \in H, u \in M_{n, 1}(\mathbb{C})
\end{array}\right\}
$$

où $H$ est un sous-groupe du groupe orthogonal, $K$ un sous-groupe de $\mathbb{C}^{*}$. On a, pour tout $g \in G$, la relation ${ }^{t} g A g=A$ avec :

$$
A=\left(\begin{array}{ccc}
a & 0 & -k^{-1} \\
0 & \operatorname{Id}_{n} & 0 \\
-k^{-1} & 0 & 0
\end{array}\right)
$$

où $a$ est une constante quelconque et réciproquement si $A$ vérifie, pour tout $g \in G$, la relation ${ }^{t} g A g=A$; alors il existe une constante $a$ telle que $A$ ait la forme précédente.

Démonstration. - Comme dans le lemme précédent. Posons :

$$
A=\left(\begin{array}{lll}
a & a^{\prime} & a^{\prime \prime} \\
b & b^{\prime} & b^{\prime \prime} \\
c & c^{\prime} & c^{\prime \prime}
\end{array}\right)
$$

avec $a, a^{\prime \prime}, c, c^{\prime \prime} \in \mathbb{C}, b^{\prime} \in M_{n, n}(\mathbb{C}), b, b^{\prime \prime} \in M_{n, 1}(\mathbb{C}), a^{\prime}, c^{\prime} \in M_{1, n}(\mathbb{C})$. En écrivant : ${ }^{t} M A M=A$ pour tout $M$ appartenant à $G$, on obtient les relations :

- ${ }^{t} \sigma b^{\prime \prime}+v c^{\prime \prime}=b^{\prime \prime}$ pour tout $\sigma \in H$ et $v \in M_{n, 1}(\mathbb{C})$, d'où $b^{\prime \prime}=0$ et $c^{\prime \prime}=0$;

- $c^{\prime} \sigma=c^{\prime}$ pour tout $\sigma \in H$, d'où $c^{\prime}=0$;

- ${ }^{t} \sigma b^{\prime} \sigma=b^{\prime}$ pour tout $\sigma \in H$; d'après le lemme A.2, comme $H$ opère irréductiblement sur $\mathbb{C}^{n}$, il existe une constante $\lambda \neq 0$ telle que $b^{\prime}=\lambda \operatorname{Id}_{n}$;

- $a^{\prime} \sigma+{ }^{t} u b^{\prime} \sigma+a^{\prime \prime t} v=a^{\prime}$ pour tout $\sigma \in H$ et $v \in M_{n, 1}(\mathbb{C})$; 
- ${ }^{t} \sigma b+v c+{ }^{t} \sigma b^{\prime} u=b$ pour tout $\sigma \in H$ et $v \in M_{n, 1}(\mathbb{C})$; en transposant cette relation, on obtient :

$$
{ }^{t} b \sigma+{ }^{t} u b^{\prime} \sigma+c^{t} v={ }^{t} b \quad \text { pour tout } \sigma \in H \text { et } v \in M_{n, 1}(\mathbb{C}) \text {; }
$$

En retranchant les relations (1) et (2), on a $\left(a^{\prime}-{ }^{t} b\right) \sigma+\left(a^{\prime \prime}-c\right)^{t} v=$ $a^{\prime}-{ }^{t} b$ pour tout $\sigma \in H$ et $v \in M_{n, 1}(\mathbb{C})$, d'où :

$$
a^{\prime}={ }^{t} b
$$

et

$$
a^{\prime \prime}=c
$$

- $a+{ }^{t} u b+x c+a^{\prime} u+{ }^{t} u b^{\prime} u+a^{\prime \prime} x=a$ pour tout $u \in M_{n, 1}(\mathbb{C})$.

D'où, en tenant compte des relations (3), (4) et $b^{\prime}=\lambda \operatorname{Id}_{n}$ :

$$
2^{t} b u+2 x c+\lambda^{t} u u=0 .
$$

Comme $A$ n'est pas dégénérée, $c$ est une constante non nulle et si l'on pose

$$
B=\frac{-1}{c} t_{b} \text { et } C=\frac{-\lambda}{c},
$$

on obtient, d'après les relations (1) et (5) :

$$
{ }^{t} v=B(\sigma-1)+C^{t} u \sigma \quad \text { et } \quad x=B u+C \frac{{ }^{t} u u}{2} .
$$

Le lemme se déduit de ces deux relations.

\section{Remerciements}

Je tiens à remercier le referee pour ses remarques sur une version préliminaire de l'article.

\section{Bibliographie}

[BBH] Beukers (F.), Brownawell (W. D.) et Heckmann (G.) - - Siegel Normality, Annals of Math. 127 (1988), pp. 279-308.

[BH] Beukers (F.) et Heckmann (G.) .- Monodromy for the hypergeometric function ${ }_{n} F_{n-1}$, Inv. Math. 95 (1989), pp. 325-354. 
[Be1] BERTRAND (D.) .- Groupes algébriques linéaires et théorie de Galois différentielle, Cours de 3ème cycle, Université Pierre et Marie-Curie, Notes rédigées par R. Lardon (1985-86).

[Be] Bertrand (D.) .- Extensions de D-modules et groupes de Galois différentiels, Lecture Notes in Math., Springer, 1454 (1990), pp. 125-141.

[Be2] Bertrand (D.) .- Un analogue différentiel de la théorie de Kummer, Conf. de Luminy, P. Philippon (éd.), W. de Gruyter (1992).

[Bo] Bourbaki (N.) .- Algèbre, Hermann, Paris (1970).

[Bou] Boussel (K.) .- Groupes de Galois des équations hypergéométriques [...], CRAS Paris 309 (1989), pp. 587-589.

[Bou1] Boussel (K.) .- Groupes de Galois des équations hypergéométriques [...], Thèse, Université de Paris-VI (1992).

[De] Deligne (P.) .- Equations différentielles à points singuliers réguliers, Lecture Notes in Math., Springer 163 (1970).

[DuMi] Duval (A.) et Mitschi (C.) .- Matrices de Stokes et groupes de Galois des équations hypergéométriques confluentes généralisées, Pacific J. Math. 138 (1989), pp. 25-56.

[K] KAPLANSKY (I.) .- An Introduction to differential algebra, Hermann, Paris (1957-1976).

[Ka1] KatZ (N. M.) .- On the calculation of some differential Galois groups, Invent. Math. 87 (1987), pp. 13-61.

[Ka] Katz (N. M.) .- Exponential sums and differential equations, Princeton University Press (1991).

[Ko] Kolchin (E. R.) .- Differential Algebra and Algebraic Groups, Academic Press, New-York (1973).

[LoSa] Loeser (F.) et SABBAH (C.) .- Caractérisation des D-modules hypergéométriques sur le tore, CRAS Paris 312 (1991), pp. 735-738.

[Ma] MALGRANGE (B.) .- - Sur la réduction formelle des équations différentielles à singularités irrégulières, Preprint, Université de Grenoble (1979).

[MR] Martinet (J.) et RAmis (J.-P.) . - Théorie de Galois différentielle et resommation, Computer Algebra and Differential Equations, E. Tournier (éd.), Acad. Press, (1989).

[Mi] Mitschi (C.) . - Groupes de Galois et G-fonctions, Institut de Recherche Mathématique Avancée (Thèse, 1989); Computer Algebra and Diff. Eqs, M. Singer (éd.), Acad. Press (1991).

[MnTe] MNeimé (R.) et Testard (F.) .- Introduction à la théorie des groupes de Lie classiques, Hermann, Paris (1986).

[Po] POOLE (E.) .- Introduction to the theory of linear differential equations, Dover (1960).

[Ro] RobBa (P.) .- Lemmes de Hensel pour les opérateurs différentiels, Ens. Math. 26 (1980), pp. 279-311.

[Se] Serre (J.-P.) - - Corps locaux, Hermann, Paris (1962). 